\title{
Math Active Learning Lab: Math 98 Notebook
}

\author{
Gwennie Byron \\ University of North Dakota, gwennie.byron@UND.edu \\ Michele liams \\ University of North Dakota, michele.iiams@UND.edu \\ Department of Mathematics, University of North Dakota
}

How does access to this work benefit you? Let us know!

Follow this and additional works at: https://commons.und.edu/oers

Part of the Algebra Commons

\section{Recommended Citation}

Byron, Gwennie; liams, Michele; and Department of Mathematics, University of North Dakota, "Math Active Learning Lab: Math 98 Notebook" (2020). Open Educational Resources. 25.

https://commons.und.edu/oers/25

This Course Material is brought to you for free and open access by UND Scholarly Commons. It has been accepted for inclusion in Open Educational Resources by an authorized administrator of UND Scholarly Commons. For more information, please contact und.commons@library.und.edu. 


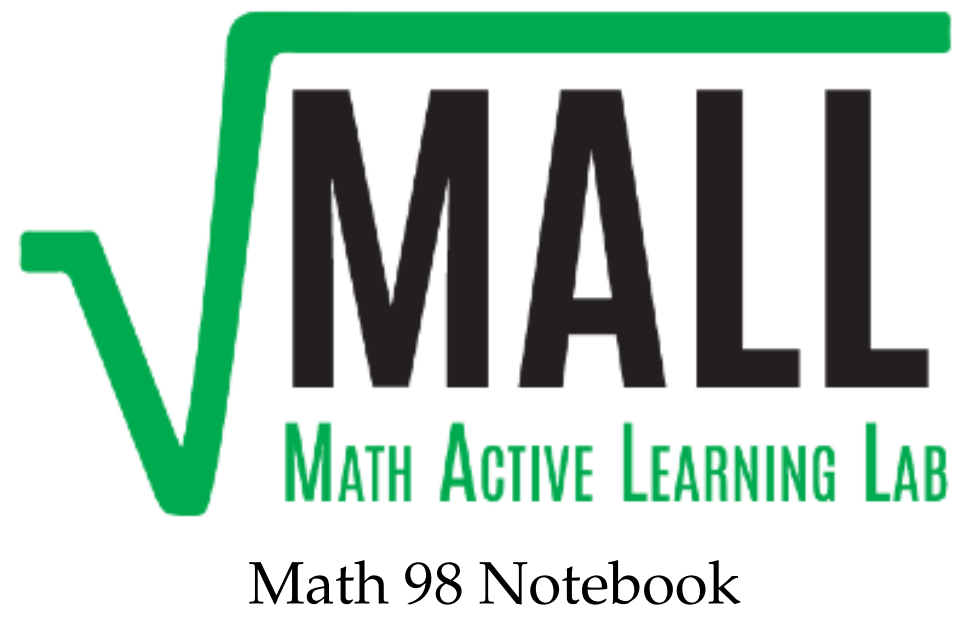

University of North Dakota

Revised August 2020 
(C) 2019

Department of Mathematics

University of North Dakota

(c) (1) (2) This work is licensed under a

BY No sa Creative Commons

Attribution-NonCommercial-ShareAlike 4.0 International License. 


\section{Welcome to the MALL}

\section{$\sqrt{\text { MALL }}$}

Welcome to UND's Math Active Learning Lab (MALL)! The MALL is a research-based approach designed to support student engagement with math. The premise of the MALL is that the best way to learn math is by doing math, not by watching someone else do math. This means that most of your time in this course will be spent doing math with the MALL instructors and tutors available to support your learning. The philosophy of the MALL is well described by H. A. Simon's quote

"Learning results from what the student does and thinks and ONLY from what the student does and thinks. The teacher can advance learning only by influencing what the student does to learn."

For many of you, this is your first college math course. Quite possibly, this course and our expectations may be different from your high school mathematics experiences. We cannot stress strongly enough your role in ensuring your success in this class. More than anything else, your choices will determine your success in this course.

- Attending class (focus group) regularly,

- diligently working in ALEKS and this Notebook,

- studying for exams, and

- seeking help when you need it

will lead to success. We will be asking you to use the ALEKS resources and to work in your notebooks before coming to class. In your weekly focus group, your instructor will support your learning by facilitating smallgroup assignments and providing mini-lectures on the more challenging topics.

Instead of sitting in a lecture class for hours each week AND then being expected to do practice problems outside of class, part of your "class time" is spent doing homework in ALEKS. This provides instant feedback and links you to resources as needed. Using ALEKS allows us to individualize the student learning path. Students can move quickly through topics they are familiar with and take the time they need to learn more challenging topics. To help you get the most out of ALEKS, we have created this notebook. If ALEKS and the notebook are still leaving you confused about a topic, we expect you to ask an instructor or tutor for help.

MALL Staff 


\section{Contents}

$\begin{array}{ll}\text { How to use ALEKS } & 10\end{array}$

Working in ALEKS with the Notebook . . . . . . . . . . . . . . . . . . . . 10

$\square$ The Learning Carousel . . . . . . . . . . . . . . . . . . . . . . . . . . . 10

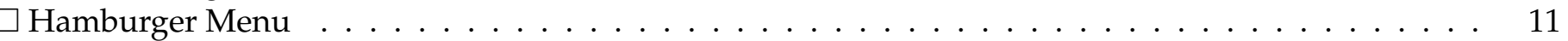

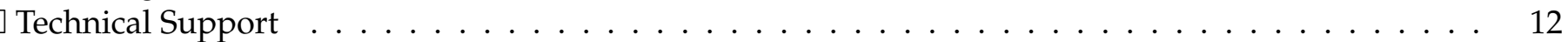

$\begin{array}{ll}\text { Syllabus } & 13\end{array}$

Time Management $\quad 19$

$\begin{array}{lr}\text { Test Analysis } & 22\end{array}$

Module 1 2

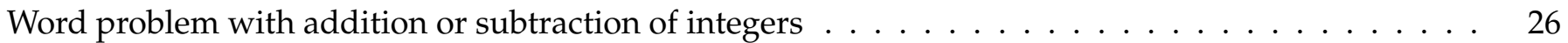

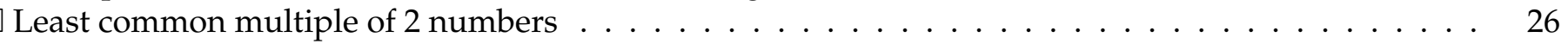

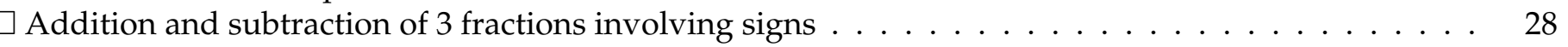

$\square$ Signed fraction division . . . . . . . . . . . . . . . . . . . . . . . . . . . . 29

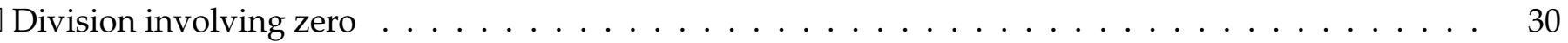

Exponents and integers: Problem type $1 \ldots \ldots \ldots \ldots \ldots \ldots \ldots \ldots \ldots \ldots$

$\square$ Exponents and integers: Problem type $2 \ldots \ldots \ldots \ldots \ldots \ldots \ldots \ldots \ldots$

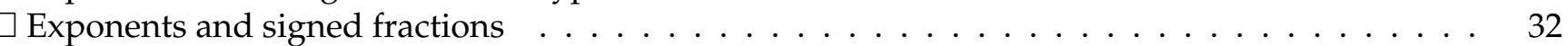

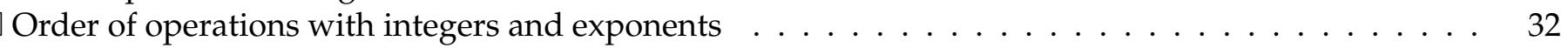

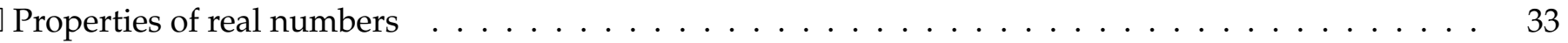

$\square$ Distributive property: Integer coefficients . . . . . . . . . . . . . . . . . . . . 34

$\square$ Using distribution with double negation and combining like terms to simplify: Multivariate . . . . 34

$\square$ Additive property of equality with signed fractions . . . . . . . . . . . . . . . . 35

$\square$ Multiplicative property of equality with signed fractions . . . . . . . . . . . . . . 36

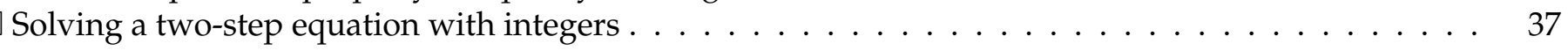

$\square$ Solving a two-step equation with signed fractions . . . . . . . . . . . . . 37

$\square$ Solving a linear equation with several occurrences of the variable: Variables on both sides and distri-

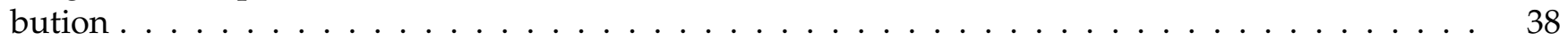

Translating a sentence into a multi-step equation $\ldots \ldots \ldots \ldots \ldots$

Writing a multi-step equation for a real-world situation . . . . . . . . . . . . . . . . . . . 40

Writing a one-step expression for a real-world situation . . . . . . . . . . . . . . . . . . 41

$\square$ Solving a word problem with two unknowns using a linear equation . . . . . . . . . . . . . 42

Module 2

Writing an inequality for a real-world situation $\ldots \ldots \ldots \ldots \ldots$

$\square$ Writing an inequality given a graph on the number line . . . . . . . . . . . . . 46

$\square$ Graphing a linear inequality on the number line . . . . . . . . . . . . . . . . 47

$\square$ Graphing a compound inequality on the number line . . . . . . . . . . . . 48 
Set-builder and interval notation . . . . . . . . . . . . . . . . . . . . . . . 49

$\square$ Evaluating a quadratic expression: Integers . . . . . . . . . . . . . . . . . . 5 50

$\square$ Solving for a variable in terms of other variables using addition or subtraction: Advanced . . . . . 50

$\square$ Solving for a variable in terms of other variables using addition or subtraction with division . . 51

$\square$ Solving for a variable inside parentheses in terms of other variables . . . . . . . . . . . 51

Additive property of inequality with signed fractions . . . . . . . . . . . . . . . . . 51

Writing a compound inequality given a graph on the number line . . . . . . . . . . . 52

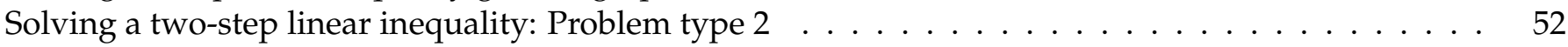

Multiplicative property of inequality with integers $\ldots \ldots \ldots \ldots \ldots$

$\square$ Solving a compound linear inequality: Graph solution, basic . . . . . . . . . . . . . . . . . . 54

$\square$ Solving a compound linear inequality: Interval notation . . . . . . . . . . . . . . 56

Introduction to solving an absolute value equation . . . . . . . . . . . . . . . 57

$\square$ Solving an absolute value equation: Problem Type $1 \ldots \ldots \ldots \ldots \ldots \ldots$

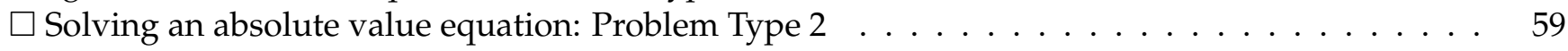

$\square$ Solving inequalities with no solution or all real numbers as solutions . . . . . . . . . . . . . 60

$\square$ Translating a sentence into a multi-step inequality . . . . . . . . . . . . . 61

$\begin{array}{ll}\text { Module } 3 & \mathbf{6 4}\end{array}$

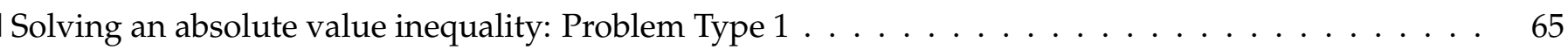

$\square$ Solving an absolute value inequality: Problem Type $2 \ldots \ldots \ldots \ldots 6$

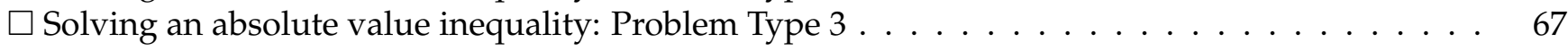

Writing an absolute value inequality given a graph on the number line . . . . . . . . . . . 68

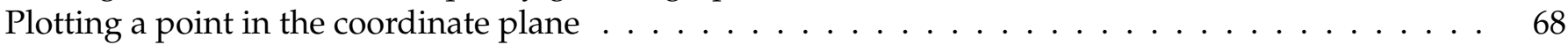

Identifying solutions to a linear equation in two variables . . . . . . . . . . . . . . . . 69

$\square$ Graphing a line given its equation in slope-intercept form: Integer slope . . . . . . . . . . . . . . . 70

$\square$ Graphing a line given its equation in slope-intercept form: Fractional slope . . . . . . . . 71

$\square$ Graphing a vertical or horizontal line . . . . . . . . . . . . . . . . 71

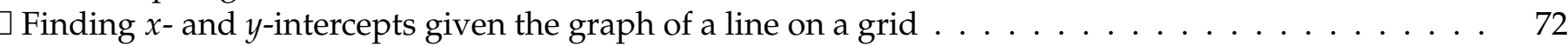

$\square$ Finding $x$ - and $y$-intercepts given the graph of a line given the equation: Basic . . . . . . 74

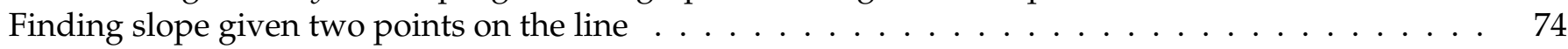

$\square$ Finding the slope of horizontal and vertical lines $\ldots \ldots \ldots \ldots \ldots$

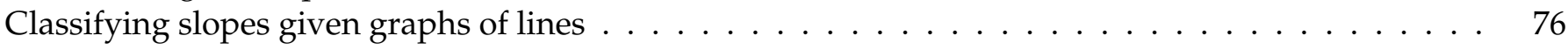

$\square$ Graphing a line through a given point with a given slope . . . . . . . . . . . . . . 77

$\square$ Graphing a line by first finding its slope and y-intercept . . . . . . . . . . . . 78

Finding the slope and $y$-intercept of a line given its equation in the form $y=m x+b \ldots \ldots 79$

Writing an equation in slope-intercept form given the slope and a point . . . . . . . . . . . . 79

$\square$ Writing the equations of vertical and horizontal lines through a given point $\ldots \ldots \ldots$. . . . . 80

$\square$ Writing the equation of the line through two given points $\ldots \ldots \ldots$. . . . . . . . . 81

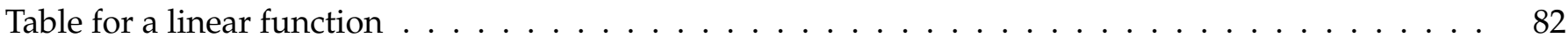

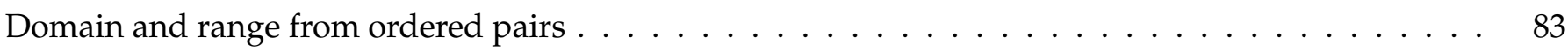

$\begin{array}{lr}\text { Module 4-Review } & 86\end{array}$

Module 5

$\square$ Square root of a perfect square with signs $\ldots \ldots \ldots \ldots \ldots$

$\square$ Domain and range from the graph of a continuous function . . . . . . . . . . . . . . 89

$\square$ Domain of a square root function: Basic . . . . . . . . . . . . . . . . . . . . 90

Identifying functions from relations $\ldots \ldots \ldots \ldots \ldots \ldots \ldots \ldots \ldots$

$\square$ Evaluating functions: Linear and quadratic or cubic . . . . . . . . . . . . . . . . . . . . . 91

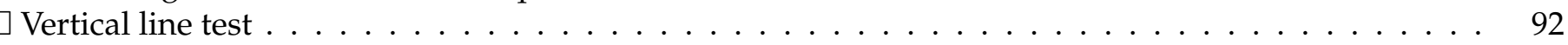

Variable expressions as inputs of functions: Problem type $1 \ldots \ldots \ldots \ldots$ 
Finding outputs of a one-step function that models a real-world situation: Function notation . . . . 93

$\square$ Finding inputs and outputs of a function from its graph . . . . . . . . . . . . . . . . 94

$\square$ Identifying solutions to a system of linear equations . . . . . . . . . . . . . . . . . . . . . . . . . . . . . . .

$\square$ Graphically solving a system of linear equations . . . . . . . . . . . . . . . . . . . 96

$\square$ Solving a system of linear equations using substitution . . . . . . . . . . . . . . . . . 97

$\square$ Solving a system of linear equations using elimination with addition . . . . . . . . . . . . . 99

$\square$ Solving a system of linear equations using elimination with multiplication and addition _. . . 100

Solving a word problem involving a sum and another basic relationship using a system of linear

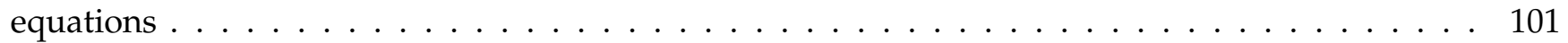

Solving a word problem using a system of linear equations of the form $A x+B y=C \ldots \ldots 2$

$\square$ Solving a value mixture problem using a system of linear equations . . . . . . . . . . . . 103

$\square$ Solving a distance, rate, time problem using a system of linear equations . . . . . . . . . . . 104

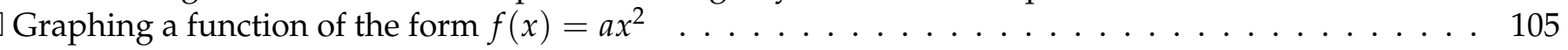

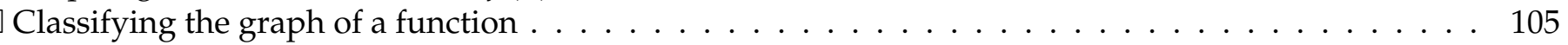

Interpreting the graphs of two functions $\ldots \ldots \ldots \ldots \ldots \ldots \ldots$

$\begin{array}{ll}\text { Module } 6 & 108\end{array}$

Understanding the product rule of exponents f . . . . . . . . . . . . . . . . . . . 109

$\square$ Introduction to the product rule of exponents . . . . . . . . . . . . . . . . . . 109

$\square$ Product rule with positive exponents: Univariate . . . . . . . . . . . . . . . . . . . . . . . 110

$\square$ Product rule with positive exponents: Multivariate . . . . . . . . . . . . . . . . . . 110

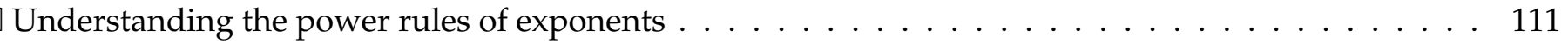

$\square$ Introduction to the power of a power rule of exponents . . . . . . . . . . . . . . . 111

$\square$ Introduction to the power of a product rule of exponents . . . . . . . . . . . . . . . 112

Introduction to the quotient rule of exponents . . . . . . . . . . . . . . . . . . . . 113

$\square$ Quotient of expressions involving exponents . . . . . . . . . . . . . . . . . . 114

$\square$ Power rules with positive exponents: Multivariate products . . . . . . . . . . . . . . . . . 114

$\square$ Power and quotient rules with positive exponents . . . . . . . . . . . . . . . . . 115

$\square$ Evaluating expressions with exponents of zero . . . . . . . . . . . . . . . . 116

$\square$ Evaluating an expression with a negative exponent: Whole number base . . . . . . . . . . 117

$\square$ Evaluating an expression with a negative exponent: Negative integer base . . . . . . . . . . 117

$\square$ Evaluating an expression with a negative exponent: Positive fraction base . . . . . . . . . . . 118

$\square$ Rewriting an algebraic expression without a negative exponent . . . . . . . . . . . . . . . . . . . . . . . . . . . .

Introduction to the product rule with negative exponents . . . . . . . . . . . . . . . . 120

$\square$ Product rule with negative exponents . . . . . . . . . . . . . . . . . . . . . 120

$\square$ Quotient rule with negative exponents: Problem type $1 \ldots \ldots$. . . . . . . . . . . . . 121

$\square$ Power of a power rule with negative exponents . . . . . . . . . . . . . . . . . . 122

$\begin{array}{lr}\text { Module } 7 & 124\end{array}$

$\square$ Degree and leading coefficient of a univariate polynomial . . . . . . . . . . . . . . . . . . . . 125

$\square$ Simplifying a sum or difference of two univariate polynomials . . . . . . . . . . . . . . . . . 125

$\square$ Multiplying a univariate polynomial by a monomial with a negative coefficient . . . . . . . . . 126

$\square$ Multiplying a multivariate polynomial by a monomial . . . . . . . . . . . . . . . 127

$\square$ Multiplying binomials with leading coefficients of $1 \ldots \ldots \ldots \ldots \ldots$

$\square$ Multiplying binomials with leading coefficients greater than $1 \ldots \ldots \ldots \ldots$

$\square$ Multiplying binomials with negative coefficients . . . . . . . . . . . . . . . . . 128

Multiplication involving binomials and trinomials in one variable . . . . . . . . . . . . . . . 129

Multiplying conjugate binomials: Univariate . . . . . . . . . . . . . . . . . . . . 130

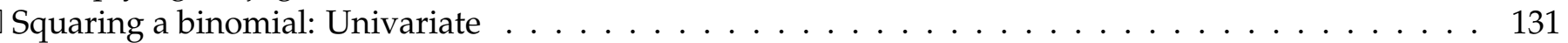

Dividing a polynomial by a monomial: Univariate . . . . . . . . . . . . . . . . . . . . 132

$\square$ Polynomial long division: Type $1 \ldots \ldots \ldots \ldots \ldots \ldots \ldots \ldots$ 
Polynomial long division: Type $2 \ldots \ldots \ldots \ldots \ldots \ldots$. . . . . . . . . . . . . . 134

Greatest common factor of 2 numbers . . . . . . . . . . . . . . . . . . . . . 135

$\square$ Greatest common factor of three univariate monomials . . . . . . . . . . . . . . . . 136

$\square$ Greatest common factor of two multivariate monomials . . . . . . . . . . . . . 137

Factoring out a monomial from a polynomial: Univariate . . . . . . . . . . . . . . . . 138

Factoring out a binomial from a polynomial: GCF factoring, basic . . . . . . . . . . . . . 138

Factoring a univariate polynomial by grouping: Problem type $1 \ldots \ldots$. . . . . . . . . . . . 139

$\square$ Factoring a univariate polynomial by grouping: Problem type $2 \ldots \ldots$. . . . . . . . . . 140

Module 8-Review

$\begin{array}{ll}\text { Module } 9 & 143\end{array}$

$\square$ Factoring a quadratic with leading coefficient $1 \ldots \ldots \ldots \ldots \ldots \ldots$

$\square$ Factoring a quadratic with a negative leading coefficient . . . . . . . . . . . . . . . . 144

$\square$ Factoring out a constant before factoring a quadratic . . . . . . . . . . . . . . . . . 145

$\square$ Factoring a quadratic with leading coefficient greater than 1 : Problem type $1 \ldots \ldots$. . . . . . . . 146

$\square$ Factoring a quadratic with leading coefficient greater than 1: Problem type $2 \ldots \ldots$. . . . 146

Factoring a perfect square trinomial with leading coefficient $1 \ldots \ldots \ldots \ldots \ldots$

$\square$ Factoring a perfect square trinomial with leading coefficient greater than $1 \ldots \ldots$. . . . . 149

Factoring a product of a quadratic trinomial and a monomial . . . . . . . . . . . . . . 149

$\square$ Factoring a difference of squares in one variable: Basic . . . . . . . . . . . . . . . . . . . . . 150

$\square$ Factoring a difference of squares in one variable: Advanced . . . . . . . . . . . . . . 151

Solving an equation written in factored form . . . . . . . . . . . . . . . . . . . 152

$\square$ Finding the roots of a quadratic equation with leading coefficient $1 \ldots \ldots \ldots \ldots$

$\square$ Finding the roots of a quadratic equation of the form $a x^{2}+b x=0 \ldots \ldots \ldots$

$\square$ Finding the roots of a quadratic equation with leading coefficient greater than $1 \ldots \ldots 154$

Solving a quadratic equation needing simplification . . . . . . . . . . . . . . . . . . 155

Solving a word problem using a quadratic equation with rational roots . . . . . . . . . . . . . 156

$\square$ Writing a quadratic equation given the roots and the leading coefficient . . . . . . . . . . . 157

$\begin{array}{lr}\text { Module } 10 & 159\end{array}$

Evaluating a rational function: Problem type $1 \ldots \ldots \ldots \ldots$. . . . . . . . . . . . . 160

Restriction on a variable in a denominator: Quadratic . . . . . . . . . . . . . . . . . . 161

$\square$ Simplifying a ratio of factored polynomials: Linear factors . . . . . . . . . . . . . . . . . 162

$\square$ Simplifying a ratio of polynomials using GCF factoring . . . . . . . . . . . . . . . . . . . . . . . . . . . . . . . .

$\square$ Simplifying a ratio of linear polynomials: $1,-1$, and no simplification . . . . . . . . . . . 163

$\square$ Simplifying a ratio of polynomials by factoring a quadratic with leading coefficient $1 \ldots \ldots$

$\square$ Simplifying a ratio of polynomials: Problem type $1 \ldots \ldots \ldots \ldots$

Multiplying rational expressions involving multivariate monomials . . . . . . . . . . . . . 166

$\square$ Multiplying rational expressions made up of linear expressions . . . . . . . . . . . . . . 167

Dividing rational expressions involving linear expressions . . . . . . . . . . . . . . . 167

$\square$ Dividing rational expressions involving quadratics with leading coefficients of $1 \ldots \ldots$

Introduction to the LCM of two monomials . . . . . . . . . . . . . . . . . . . 169

Writing equivalent rational expressions with monomial denominators . . . . . . . . . . . . . . . 169

Finding the LCD of rational expression with linear denominators: Relatively prime . . . . . . . . . . 170

Adding rational expressions with common denominators and GCF factoring . . . . . . . . . . . 171

Adding rational expressions with linear denominators without common factors: Basic . . . . 172

Adding rational expressions with linear denominators with common factors: Basic $\ldots . . . .173$

Adding rational expressions with denominators $a x^{n}$ and $b x^{m} \ldots \ldots \ldots \ldots \ldots$

Complex fraction without variables: Problem type $1 \ldots \ldots$. . . . . . . . . . . . . 175

$\square$ Complex fraction without variables: Problem type $2 \ldots \ldots \ldots \ldots \ldots$ 


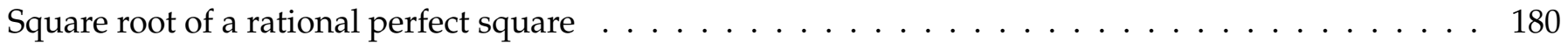

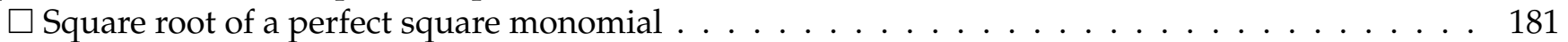

$\square$ Complex fraction: GCF factoring . . . . . . . . . . . . . . . . . . . . . . 181

$\square$ Complex fraction made of sums involving rational expressions: Problem type 1 . . . . . . . 182

$\square$ Solving a rational equation that simplifies to linear: Denominators $a, x$, or $a x \ldots \ldots \ldots$

$\square$ Solving a rational equation that simplifies to linear: Denominators $a x$ and $b x \ldots \ldots 184$

$\square$ Solving a rational equation that simplifies to linear: Denominator $x+a \ldots \ldots$

$\square$ Solving a rational equation that simplifies to quadratic: Denominator $x \ldots \ldots$. . . . . . 186

$\square$ Solving a rational equation that simplifies to quadratic: Proportional form, basic . . . . . . . . 187

Solving for a variable in terms of other variables in a rational equation: Problem type 1 . . . . . . 188

Solving a proportion of the form $\frac{(x+a)}{b}=\frac{c}{d} \ldots \ldots \ldots \ldots \ldots \ldots \ldots$

Word problem on proportions: Problem type $1 \ldots \ldots \ldots$. . . . . . . . . . . . . . . . . . 189

$\square$ Word problem on proportions: Problem type $2 \ldots \ldots \ldots$. . . . . . . . . . . . . . . 190

$\square$ Finding all square roots of a number . . . . . . . . . . . . . . . . . . . . . . . 190

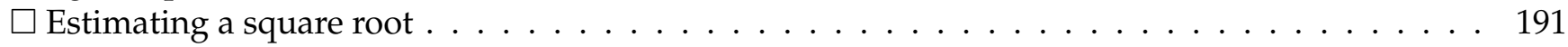

$\square$ Cube root of an integer . . . . . . . . . . . . . . . . . . . . . . . . 191

Introduction to simplifying a radical expression with an even exponent . . . . . . . . . . . . . 192

Table for a square root function . . . . . . . . . . . . . . . . . . . . . . . . . 192

Word problem involving multiple rates . . . . . . . . . . . . . . . . . . . . . . . 193

$\square$ Graphing a square root function: Problem type $1 \ldots \ldots$. . . . . . . . . . . . . . . . 194

$\begin{array}{ll}\text { Module 12-Review } & 196\end{array}$

$\begin{array}{ll}\text { Module } 13 & 197\end{array}$

$\square$ Finding $n^{\text {th }}$ roots of perfect $n^{\text {th }}$ powers with signs $\ldots \ldots \ldots \ldots$

$\square$ Finding the $n^{\text {th }}$ root of perfect $n^{\text {th }}$ power monomial $\ldots \ldots \ldots \ldots \ldots$

Converting between radical form and exponent form . . . . . . . . . . . . . . . . . . 200

$\square$ Rational exponents: Unit fraction exponents and whole number bases . . . . . . . . . . . . . . 201

$\square$ Rational exponents: Unit fraction exponents and bases involving signs . . . . . . . . . . . . . 201

$\square$ Rational exponents: Non-unit fraction exponent with a whole number base . . . . . . . . . . 202

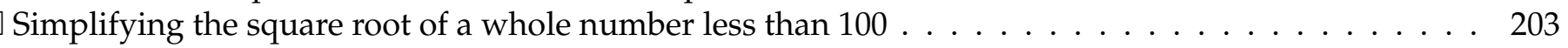

$\square$ Simplifying the square root of a whole number greater than $100 \ldots \ldots \ldots \ldots$

$\square$ Rational exponents: Product rule . . . . . . . . . . . . . . . . . . . . . . . . . . . 204

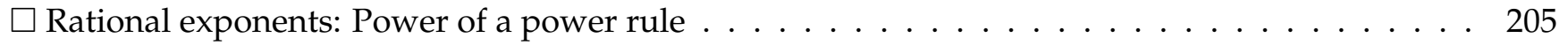

$\square$ Simplifying a radical expression with an even exponent . . . . . . . . . . . . . . . . 205

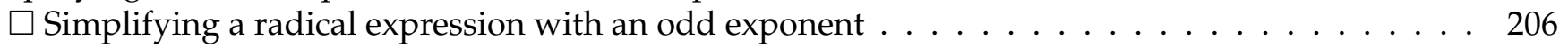

Simplifying a higher root of a whole number . . . . . . . . . . . . . . . . . 207

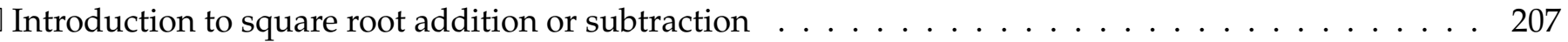

$\square$ Square root addition or subtraction . . . . . . . . . . . . . . . . . . 208

$\square$ Introduction to simplifying a sum or difference of radical expressions: Univariate . . . . . . . . 209

Simplifying a product involving square roots using the distributive property: Basic . . . . . . . . . 209

$\square$ Square root multiplication: Basic . . . . . . . . . . . . . . . . . . . . . . . . . . . 210

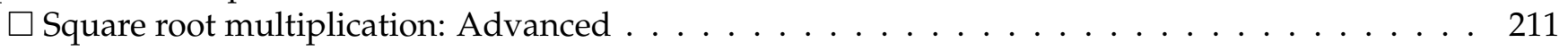

Simplifying a product of radical expressions: Univariate . . . . . . . . . . . . . . . . . . . 212

Module 14
214

$\square$ Simplifying a quotient of square roots . . . . . . . . . . . . . . . . . . . . . 215

$\square$ Introduction to solving a radical equation . . . . . . . . . . . . . . . . . . . . . 216

$\square$ Solving a radical equation that simplifies to a linear equation: One radical, basic . . . . . . . . 217

$\square$ Solving a radical equation that simplifies to a linear equation: One radical, advanced . . . . . 218 
Solving a radical equation that simplifies to a quadratic equation: One radical, basic . . . . . . . . 219

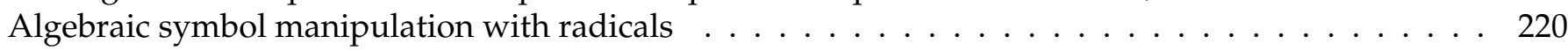

$\square$ Word problem involving radical equations: Advanced . . . . . . . . . . . . . . . . . 221

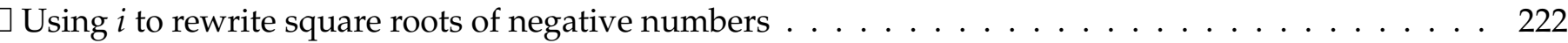

$\square$ Solving an equation of the form $x^{2}=a$ using the square root property $\ldots \ldots \ldots 222$

$\square$ Solving a quadratic equation using the square root property: Exact answers, basic . . . . . . . . . 223

$\square$ Solving a quadratic equation using the square root property: Exact answers, advanced . . . . 224

Applying the quadratic formula: Exact answers . . . . . . . . . . . . . . . . . . . . 225

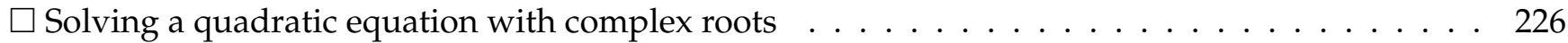

$\square$ Domain and range from the graph of a parabola . . . . . . . . . . . . . . . . . . . . 227

$\square$ How the leading coefficient affects the shape of the parabola . . . . . . . . . . . . . . 228

$\square$ Finding the vertex, intercepts, and axis of symmetry from the graph of a parabola . . . . . . . . . . 229

$\square$ Finding the $x$-intercept(s) and the vertex of a parabola . . . . . . . . . . . . . . . . . 229

$\square$ Graphing a parabola of the form $y=x^{2}+b x+c \ldots \ldots \ldots \ldots \ldots \ldots \ldots$

$\square$ Graphing a parabola of the form $y=a x^{2}+b x+c$ : Integer coefficients . . . . . . . . . 232

$\begin{array}{ll}\text { Module 15-Final Review } & 234\end{array}$

$\begin{array}{lr}\text { Solutions } & 235\end{array}$

Index
In

Common Properties, Graphs \& Formulas $\quad 243$ 


\section{How to use ALEKS}

\section{Working in ALEKS with the Notebook}

- Every ALEKS topic is in the Notebook.

- Not every topic in the Notebook will be in YOUR Learning Carousel.

- If you have already mastered a topic, you will not see the topic in your Learning Carousel.

- You do NOT need to complete the Notebook for a topic you have already mastered.

- How to work through ALEKS topics

1. ALEKS presents you with a topic.

2. Use the table of contents to find the topic in the Notebook.

3. You will find one of the following icons to help direct your learning.

$\circ \square$ Indicates you should watch a video. You may be asked to select a different video than the first video to pop up.

○ 国国 You should open the e-book.

* You may need to scrolll down to find the appropriate topic.

* Notebook entries are made to look EXACTLY like the e-book material

$\circ$ Aa Open the dictionary to show definitions of terms.

- ใ्? Directs you to resources your instructor has added.

o If there is no icon, the material should come directly from the Learning Page, which is the first page presented to you with a new topic.

\section{The Learning Carousel}

- To bring down the Learning Carousel, click the $\nabla$ on the upper left side of the ALEKS Learning page.

- $\quad$ indicates a goal topic for the current module

- 0 indicates a locked topic. Click the icon to see what topics must be worked to unlock it.

- No icon means it is a prerequisite topic. Use the Index to find the topic in your Notebook. 
- When the Learning Carousel is pulled down, you can

- Click the Filters - for options to filter topics.

- The Filter menu is shown below.

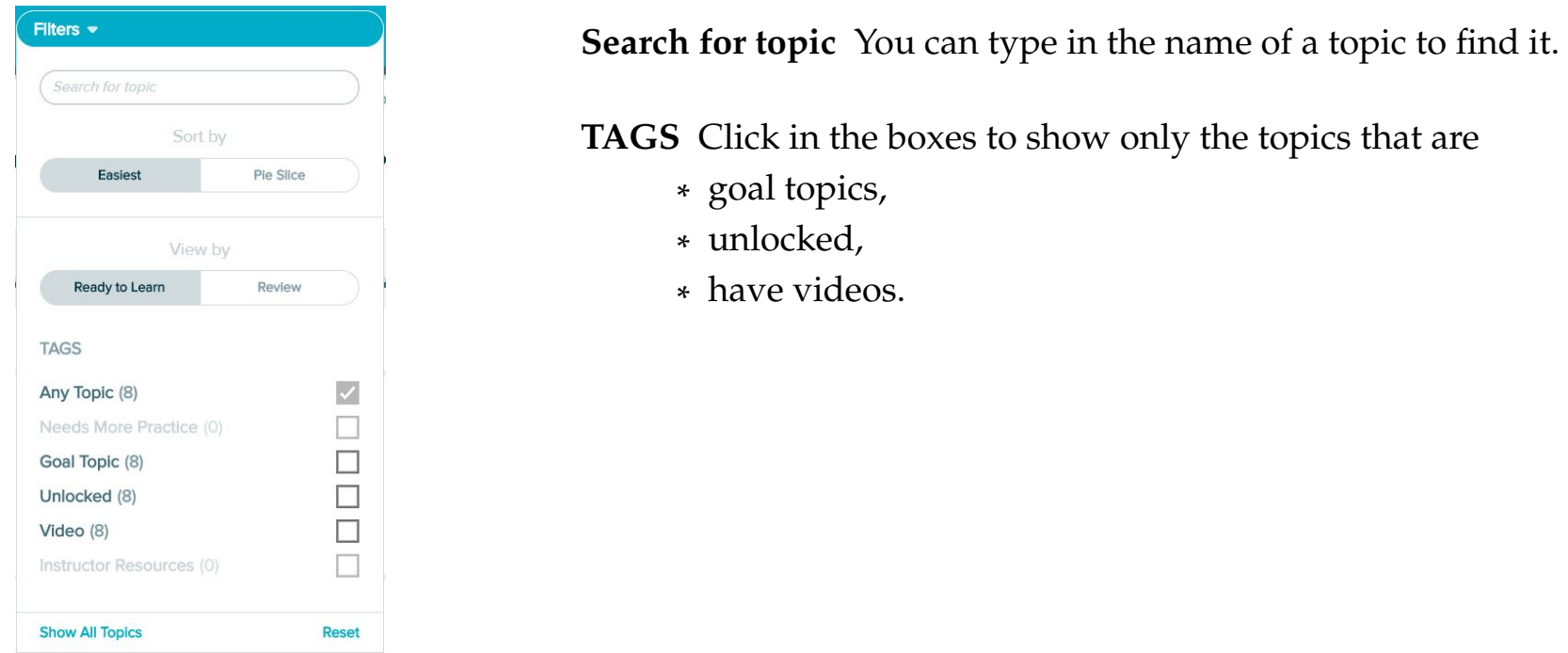

\section{Hamburger Menu}

- The Hamburger Menu $\equiv$ is in the upper left of your ALEKS screen.

- The options in the Hamburger Menu are shown below.

\begin{tabular}{l|}
\hline Home \\
\hline Learn \\
Review \\
Assignments \\
Worksheet \\
Calendar \\
Gradebook \\
Reports \\
\hline Message Center \\
\hline Instructor Resources \\
Textbook \\
Dictionary \\
\hline Manage My Classes \\
\hline
\end{tabular}

Home Takes you back to the home screen.

Learn Opens the next topic ALEKS has ready for you to learn.

Review Opens up topics you have learned or mastered for you to review.

Calendar Opens a calendar view of deadlines for weekly modules and exams.

Gradebook Shows your grades for ALEKS modules and exams. The complete and official gradebook is in Blackboard.

Reports Opens a menu of reports that provide additional information about your progress in ALEKS. We encourage you to take a look at these pages. 


\section{Technical Support}

ALEKS Technical Support is available at https://www.aleks.com/support/contact_support or by phone at (800) 258-2374. Call Technical support if you need help with

- accessing your account.

- locating a video.

- questions diplaying correctly.

- other technical issues not related to math content. 


\section{Math}

Instructor:

Phone:
Email:

Office:

\section{Focus Group:}

Required Course Materials: ALEKS 18-week access and the Course Notebook

All email correspondence will go to your official UND email address.

Course prerequisites and content: Properties of the real number system, factoring, linear and quadratic equations, functions, polynomial and rational expressions, inequalities, systems of equations, exponents, radicals. Graphing of basic functions. Study skills and problem solving techniques will be incorporated throughout the course. Does not satisfy any requirements for graduation, nor does it transfer. A grade of $\mathrm{S}$ is required in this course to be eligible to take Math 103 or Math 107.

The Math Active Learning Lab (MALL): Research shows that

, not by listening to someone talk about or present the subject. The primary reason many students do not succeed in traditional math courses is that they do not do the problems or spend enough time engaged with the material.

The MALL is a research-based approach designed to support student engagement with math. Most of your time in this course will be spent doing math, and your instructor will support your learning by facilitating in-class assignments and providing mini-lectures on the more challenging topics. Instructors and tutors are available during the required MALL time to provide just-in-time support.

In a traditional math class, all students are expected to learn at the same pace. In the MALL, the ALEKS learning system allows you to work at you own pace, skip topics you have already mastered, and provides feedback as you are working.

COVID-19: All members of the University community have a role in creating and maintaining a COVID-19 resilient campus. There are several expectations that all community members, including students, are asked to follow for the safety of all:

- maintain physical of at least 6 feet while in UND facilities,

- wear coverings during interactions with others and in the classroom,

- wash their hands often and use hand sanitizer,

- properly clean spaces that they utilize, and

- if experiencing any symptoms, and call their health care provider.

- Students electing not to comply with any of the COVID related requirements will not be permitted in the and may be subject to disciplinary action. 
All members of the University community are expected to model positive both on- and off-campus. Information regarding the pandemic and UND's efforts to create a COVID resilient campus is available on the COVID-19 blog (http://blogs.und.edu/coronavirus/). Please subscribe to stay up to date on COVID related information.

Students who test positive for COVID-19 or are identified as a close contact are expected to self-isolate/quarantine. If you have tested positive for COVID-19 or have been placed in quarantine due to being identified as a close contact or travel we strongly recommend that you report the information to the Office of Student Rights and Responsibilities at 701.777.2664 or online at https://veoci.com/veoci/p/w/ss2x4cq9238u. Doing so will ensure students have the support they need to continue with their academic goals and to protect others.

Due to the evolving circumstances of the COVID-19 pandemic, all information in this syllabus may need to be to meet the needs of remote instruction. Every effort will be made to operate in a manner consistent with the expectations outlined in this document.

\section{Course Components}

\section{Focus Group}

- Assignments given during the Focus Group meetings will be completed in small groups.

o On-time attendance is to earn full-credit on the assignment.

- Unless required for the Focus Group activity, cell-phone or computer use will result in a zero for the day.

- Students who do not attend the meeting, or contact the instructor the first week, will be DROPPED FROM THE COURSE.

- Students who do not_their Initial Knowledge Check within two full days of their first class meeting will be DROPPED FROM THE COURSE.

- Once a week you will meet in class, the other day you will work in ALEKS in the MALL or remotely.

- Focus Group Absences

- If due to a serious emergency, absences will usually be excused. Documentation

○ University sanctioned absences must be documented prior to the absence.

- Travel plans cause for an excused absence.

- All focus group assignments have a to account for any unexcused absences.

- Absences will be addressed on a case-by-case basis.

\section{ALEKS}

- Weekly module to be completed by at $11: 59 \mathrm{pm}$.

- Can work anywhere you have internet access.

- Deadlines be extended because of home computer or home internet issues. 


\section{MALL Time}

- Spend at least 2.5 hours in the MALL working in ALEKS from to

- MALL time must be completed in O'Kelly 33 (face-to-face) or virutally through Zoom.

- MALL time is class time, you should be working only on

- Credit for MALL time is based on front desk check-in/out.

- Check-in with your UND ID when entering and check-out when exiting the MALL.

- Failure to check-in/out results in _ minutes recorded.

- Check-in/out with another student's ID is academic dishonesty.

- Minutes from one week to another.

- Focus Group time toward your MALL time.

- Food is NOT allowed in the MALL.

- The MALL is the place to get your math questions answered!

- MALL staff are there

\section{Notebook}

- Graded in Focus Group.

$\bullet$ for MALL time and Focus Group.

\section{Topic Goal Extra Credit}

- Complete 10 topics in ALEKS by

- Earn a Focus Group bonus point. at 11:59 pm.

\section{Exams}

- There will be _ exams.

- Each exam will have 125 pts

○ ALEKS exam: $100 \mathrm{pts}$

* Must be completed in the MALL exam area

* Must be completed by 9:00 pm the

* UND ID is required to take your ALEKS exam.

* All scratch work must be submitted to the written exam. 30 min of test completion.

* You may not leave your table during an exam without permission.

* Cell phones must be placed face on the table.

o Written exam: 25 pts

* will be given during the Focus Group meeting.

Exam 1: Exam 2: Exam 3:

\section{Final Exam}

- The final exam will be a comprehensive ALEKS exam.

- All scratch work must be submitted to Blackboard within 30 min of test completion.

- The final ALEKS exam must be completed by Wednesday, December 16 at 7:30 pm. 


\section{Grading}

- Your course grade will be a weighted average of the following:

\begin{tabular}{ll} 
Exams & $\%$ \\
Final Exam & \multicolumn{1}{c}{$\%$} \\
MALL Time & $10 \%$ \\
Focus Group Activities & $10 \%$ \\
Module Completion & $15 \%$
\end{tabular}

- You must earn $70 \%$ or higher to get an S.

\section{Try Score}

- Your Try Score reflects your effort in this course.

- The Try Score is composed of:

○ focus group participation,

- notebook completion,

- MALL time and

- module completion.

- This is__ included in your course grade, but will be shared with your academic advisor.

\section{Finishing the Course Early}

- Given the individualized nature of this course it is possible to complete the course

- Each time an exam is given, students have the option to take the final in place of the scheduled exam.

- To qualify to take the final early

o the week before the written exam, arrange with the MALL office to take a proctored Knowledge Check

○ at least $90 \%$ of the in the course on this proctored ALEKS Knowledge

\section{Check}

\section{Academic Honesty}

- All students in attendance at the University of North Dakota are expected to be honorable and to observe standards of conduct appropriate to a community of scholars.

- Academic misconduct includes

- all acts of dishonesty in any academically related matter.

- any knowing or intentional help or attempt to help, or conspiracy to help, another student.

$\circ$ use of $\quad$, books, calculators, or any electronic devices on exams.

- A student who attempts to obtain credit for work that is not their own (whether that be on a homework assignment, exam, etc.) will receive for that item of work, and at the professor's discretion, may also receive a failing grade in the course.

- For more information read the Code of Student Life at https://und.policystat.com/ policy/6747183/latest/. 


\section{Accommodations}

- Disability

- Contact me to request disability accommodations, discuss medical information, or plan for an emergency evacuation.

○ To get confidential guidance and support for disability accommodation requests, students are expected to register with DSS at http://und.edu/disability-services/, 190 McCannel Hall, or 701.777.3425.

- COVID-19

- Due to COVID-19 students may need to request course adjustments, flexibility in delivery of content, and increased absenteeism.

- Students with concerns regarding physically attending class during COVID-19 are encouraged to do the following:

* Talk with your as soon as possible to determine appropriate accommodations,

* Students with a known disability should contact Disability Student Services (DSS).

\section{Starfish}

- Important information is available to you through Starfish, which is an online system used to help students be successful.

- When an instructor observes student behaviors or concerns that may impede academic success, the instructor may raise a flag that notifies the student of the concern and/or refer the student to their academic advisor or UND resource.

- Please pay attention to these emails and take the recommended actions. They are sent to help you be successful!

- Starfish also allows you to

o schedule appointments with various offices and individuals across campus.

○ request help on a variety of topics

o search and locate information on offices and services at UND

- You can log into Starfish by clicking on Logins on the UND homepage and then selecting Starfish. A link to Starfish is also available in Blackboard once you have signed in.

\section{Notice of Nondiscrimination}

- It is the policy of the University of North Dakota that no person shall be discriminated against because of race, religion, age, color, gender, disability, national origin, creed, sexual orientation, gender identity, genetic information, marital status, veteran's status, or political belief or affiliation and the equal opportunity and access to facilities shall be available to all.

- Concerns regarding Title IX, Title VI, Title VII, ADA, and Section 504 may be addressed to:

- Donna Smith, Director of Equal Employment Opportunity/Affirmative Action and Title IX Coordinator, 401 Twamley Hall, 701.777.4171

○ UND.affirmativeactionoffice@UND.edu

- Office for Civil Rights, U.S. Dept. of Education, 500 West Madison, Suite 1475, Chicago, IL 60611 


\section{Resolution of Problems}

Should a problem occur, you should speak to your instructor first. If the problem is not resolved, meet with Dr. Michele Iiams, MALL Director. If the problem continues to be unresolved, go to Dr. Gerri Dunnigan, Mathematics Department Chair, and next to the college Dean. Should the problem persist, you have the right to go to the Provost next, and then to the President.

\section{How to Seek Help When in Distress}

- We know that while college is a wonderful time for most students, some students may struggle.

- You may experience students in distress on campus, in your classroom, in your home, and within residence halls.

- Distressed students may initially seek assistance from faculty, staff members, their parents, and other students.

- In addition to the support we can provide to each other, there are also professional support services available to students through the Dean of Students and University Counseling Center.

- Both staffs are available to consult with you about getting help or providing a friend with the help that he or she may need.

- For more additional information, please visit the UND Cares program Webpage at https://und.edu/student-life/student-rights-responsibilities/. 


\section{Time Management}

Good time management, good study skills and good organization will help you be successful in this course (and all of your classes). Answer the following questions.

1. To motivate yourself to complete a course, it is helpful to have clear reasons for taking the course. List your goals for taking this course.

2. Taking 12-15 credit-hours is the equivalent of a full-time job. Often students try to work too many hours while taking classes.

NOTE: Students need to work to pay tuition, rent, buy food, etc., but working too many extra hours for things that are not needed can really impact their success. There is a balance between working to earn money now and having to spend more money later to retake courses.

(a) Write down the number of of credit-hours you are taking this term and the number of hours you work per week.

- Number of credit-hours

- Number of hours worked per week

(b) The table gives the recommended limit to the number of hours you should work for the number of credit-hours you are taking.

- How do your numbers from part (a) compare to those in the table?

\begin{tabular}{|c|c|}
\hline $\begin{array}{c}\text { Number of } \\
\text { Credit-Hours }\end{array}$ & $\begin{array}{c}\text { Maximum Number of Hours } \\
\text { of Work per Week }\end{array}$ \\
\hline 3 & 40 \\
\hline 6 & 30 \\
\hline 9 & 20 \\
\hline 12 & 10 \\
\hline 15 & 0 \\
\hline
\end{tabular}


(c) Keep in mind that other responsibilities in your life, such as your family, might also make it necessary to limit your hours at work even more. What other responsibilities do you have?

(d) It is often suggested that you devote 2 hours of study and homework time outside of class for each credit-hour you take. For example:

\begin{tabular}{lccc}
12 & credit-hours & 15 & credit-hours \\
24 & study hours & 30 & study hours \\
\hline 36 & total hours & 45 & total hours
\end{tabular}

- Based on the number of credit-hours you are taking, how many study hours should you plan for? credit hours $\mathrm{X} 2=$ study hours

- What is the total number of hours (class time plus study time) that you should devote to school? credit hours + study hours $=$ total hours

- Your MALL course is a 3-credit course. This means you might need to spend up to 9 hours each week in class, working in ALEKS, or studying.

- At least 2 of these hours should be completed in the MALL.

On the next page, write down the times each day (for the next week) that you

- have scheduled classes,

- are scheduled to work

- other non-negotiable commitments (family, organization meetings, etc.)

- times that you plan to work in the MALL

- times that you plan to study outside of the MALL 


\begin{tabular}{|c|c|c|c|c|c|c|c|}
\hline Time & Monday & Tuesday & Wednesday & Thursday & Friday & Saturday & Sunday \\
\hline \multicolumn{8}{|l|}{ 8:00 - 8:30 } \\
\hline \multicolumn{8}{|l|}{$8: 30$ - 9:00 } \\
\hline \multicolumn{8}{|l|}{$9: 00-9: 30$} \\
\hline \multicolumn{8}{|l|}{$9: 30-10: 00$} \\
\hline \multicolumn{8}{|l|}{ 10:00 - 10:30 } \\
\hline \multicolumn{8}{|l|}{ 10:30 - 11:00 } \\
\hline \multicolumn{8}{|l|}{ 11:00 - 11:30 } \\
\hline \multicolumn{8}{|l|}{$11: 30-12: 00$} \\
\hline \multicolumn{8}{|l|}{$12: 00$ - 12:30 } \\
\hline \multicolumn{8}{|l|}{$12: 30-1: 00$} \\
\hline \multicolumn{8}{|l|}{ 1:00 - 1:30 } \\
\hline \multicolumn{8}{|l|}{ 1:30 - 2:00 } \\
\hline \multicolumn{8}{|l|}{$2: 00$ - 1:30 } \\
\hline \multicolumn{8}{|l|}{$2: 30-3: 00$} \\
\hline \multicolumn{8}{|l|}{$3: 00-3: 30$} \\
\hline \multicolumn{8}{|l|}{$3: 30-4: 00$} \\
\hline \multicolumn{8}{|l|}{$4: 00$ - 4:30 } \\
\hline \multicolumn{8}{|l|}{$4: 30-5: 00$} \\
\hline \multicolumn{8}{|l|}{$5: 00-5: 30$} \\
\hline \multicolumn{8}{|l|}{$5: 30-6: 00$} \\
\hline \multicolumn{8}{|l|}{$6: 00-6: 30$} \\
\hline \multicolumn{8}{|l|}{$6: 30-7: 00$} \\
\hline \multicolumn{8}{|l|}{ 7:00 - 7:30 } \\
\hline \multicolumn{8}{|l|}{$7: 30$ - 8:00 } \\
\hline \multicolumn{8}{|l|}{ 8:00 - 8:30 } \\
\hline \multicolumn{8}{|l|}{$8: 30$ - 9:00 } \\
\hline \multicolumn{8}{|l|}{$9: 00-9: 30$} \\
\hline \multicolumn{8}{|l|}{$9: 30-10: 00$} \\
\hline \multicolumn{8}{|l|}{ 10:00 - 10:30 } \\
\hline $10: 30-11: 00$ & & & & & & & \\
\hline
\end{tabular}




\section{Test Analysis}

Have you ever thought of your graded test as a learning experience? There is a lot you can learn about yourself, your study habits, and your test-taking skills by examining your graded test after you get it back.

- Did you do as well as you thought you could?

- Or is there room for improvement?

You may think, "the test was too hard" or "the teacher didn't give us enough time", but, chances are, your instructor has been giving a similar test under similar conditions to many students before you. So let's see what YOU can do to earn a higher score on your next test.

Look at your graded test and analyze if each point loss was due to your having been unprepared for that problem, a concept error, or a careless error .

- Being underprepared for a problem means you didn't know how to do the problem because you hadn't done the homework that would have prepared you for it. Often an error made is considered an underprepared error if you look at the problem and have no idea where to begin.

- A concept error is one where you really didn't understand the concept behind the problem. No matter how much time was available for a problem like this, you wouldn't have been able to do it correctly because you have no conceptual understanding of the problem. This is not a procedural error: you can apply a procedure and still not understand the concept. Students demonstrate conceptual understanding in mathematics when they provide evidence that they can recognize, label, and generate examples of concepts; use and interrelate models, diagrams, manipulatives, and varied representations of concepts; identify and apply principles; know and apply facts and definitions; compare, contrast, and integrate related concepts and principles; recognize, interpret, and apply the signs, symbols, and terms used to represent concepts. Conceptual understanding reflects a student's ability to reason in settings involving the careful application of concept definitions, relations, or representations of either.

- A careless error is one where you understood the problem and knew how to solve it, but you made a mistake that could have been avoided. Maybe you copied the problem or your handwriting incorrectly, made a relatively minor mistake in calculation, or some similar error. 
1. In the chart below, put the number of points you missed on each problem under the correct heading. Then find the total in each column.

\begin{tabular}{|c|c|c|c|}
\hline Problem & unprepared & $\begin{array}{l}\text { concept } \\
\text { error }\end{array}$ & $\begin{array}{l}\text { careless } \\
\text { error }\end{array}$ \\
\hline & & & \\
\hline & & & \\
\hline & & & \\
\hline & & & \\
\hline & & & \\
\hline & & & \\
\hline & & & \\
\hline & & & \\
\hline & & & \\
\hline & & & \\
\hline & & & \\
\hline & & & \\
\hline & & & \\
\hline & & & \\
\hline & & & \\
\hline & Total points & $\begin{array}{l}\text { Total } \\
\text { points }\end{array}$ & $\begin{array}{l}\text { Total } \\
\text { points }\end{array}$ \\
\hline & & & \\
\hline
\end{tabular}

2. In which column did you have the most missed points? What does that tell you about yourself?

3. What can you learn from this exercise?

\section{Being Unprepared}

Consider the points you lost because you were unprepared. Why did you take a test without being fully prepared? Often, activities and responsibilities in life interfere with good intentions about being diligent in attending class, completing the notebook, completing MALL time, and completing the module. It may be time to:

- re-examine your weekly schedule and make sure you are devoting a sufficient amount of time to this class. Lay out a time management grid of your schedule making sure to schedule your MALL time and math study time throughout the week.

- re-commit yourself to succeeding in this class. Think about your college and career goals and remind yourself of how this course helps you get one step closer to achieving them.

4. List two steps you will take to remedy being unprepared. 


\section{Concept Errors}

Now consider the concept errors point loss. A high total in this column tells you that you didn't understand the concepts very well. You may understand a math concept for the hour you're working on problems, but forget it by the next day; possibly because you didn't do enough homework.

- Take Knowledge Checks when they appear. Knowledge Checks (KCs) are the way ALEKS helps you identify topics you are not retaining. Take each KC as if it were a QUIZ (no notebooks, calculators, friends, other websites, etc.) AND to the BEST OF YOUR ABILITY. Topics that you need to revisit will appear again in later modules as they are needed.

- Get the help you need immediately! Math concepts build on each other. Each new idea is based on many previous concepts. Make sure you get the help you need immediately, as soon as you find yourself beginning to feel lost, so that the confusion doesn't compound itself - otherwise it can become like a snowball, getting bigger and bigger as it roles through the snow.

If your total loss due to concept errors is fairly large, find out where you can get the help you need. A high concept error total is cause for concern and must be addressed immediately for you to succeed.

5. Which of the following can help you when you are struggling with math?

(a) your instructor

(b) MALL tutors

(c) Reworking and asking questions about previous Focus Group assignments

(d) Completing your Notebook pages

(e) All of the above

\section{Careless Errors}

Next look at careless error point loss. Careless errors are often caused by hurrying during a test or by lack of concentration due to test-anxiety or over-confidence. Here are some strategies that have worked for other students:

- Do the easiest problems first. When you first start a test, look it over and note which problems will be easiest for you. Do all those problems first to ensure you don't leave an easy problem blank just because it is at the end of the test. Finishing problems you find easy will help build your confidence! Then go through the rest of the test from beginning to end.

- Work carefully and neatly. As you do each problem, try to focus on one step at a time.

- Review each problem to look for careless errors when you finish the test. Find and correct common careless errors like arithmetic mistakes and sign errors before you turn in your test.

- Whenever possible, check your answer.

A lot of points can be gained by slowing down and being careful.

6. What are things you will do next time to prevent careless errors?

7. Now take half of your careless errors point total and add it back to your test total. What could your test grade have been? Would it have changed the letter grade? 


\section{Module 1}

\section{Contents}

$\square$ Word problem with addition or subtraction of integers $\ldots \ldots \ldots \ldots \ldots$

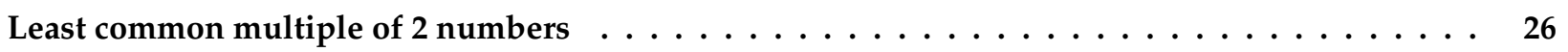

Addition and subtraction of 3 fractions involving signs $\ldots \ldots \ldots \ldots \ldots$

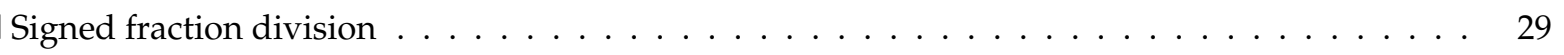

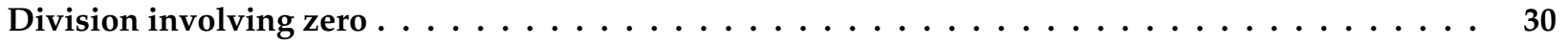

Exponents and integers: Problem type $1 \ldots \ldots \ldots \ldots \ldots \ldots \ldots \ldots \ldots$

$\square$ Exponents and integers: Problem type $2 \ldots \ldots \ldots \ldots \ldots \ldots \ldots \ldots \ldots \ldots$

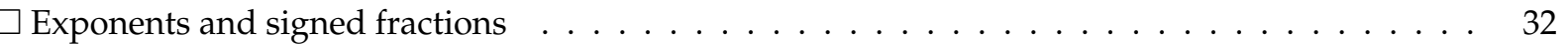

Order of operations with integers and exponents $\ldots \ldots \ldots \ldots \ldots \ldots \ldots \ldots \ldots$

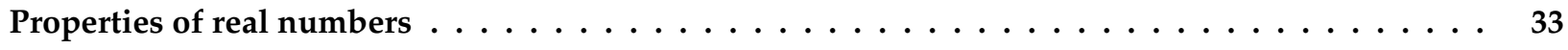

$\square$ Distributive property: Integer coefficients $\ldots \ldots \ldots \ldots \ldots \ldots \ldots \ldots \ldots \ldots \ldots$

Using distribution with double negation and combining like terms to simplify: Multivariate . . 34

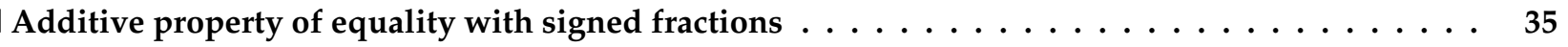

$\square$ Multiplicative property of equality with signed fractions $\ldots \ldots \ldots 36$

Solving a two-step equation with integers $\ldots \ldots \ldots \ldots \ldots \ldots \ldots \ldots \ldots$

$\square$ Solving a two-step equation with signed fractions $\ldots \ldots \ldots \ldots \ldots \ldots$

Solving a linear equation with several occurrences of the variable: Variables on both sides and

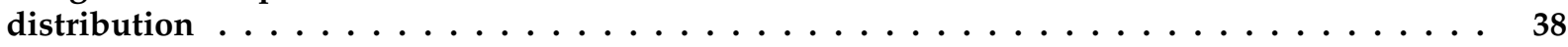

Translating a sentence into a multi-step equation $\ldots \ldots \ldots \ldots \ldots \ldots \ldots \ldots \ldots$

Writing a multi-step equation for a real-world situation $\ldots \ldots \ldots \ldots \ldots \ldots$

Writing a one-step expression for a real-world situation $\ldots \ldots \ldots \ldots \ldots \ldots$

Solving a word problem with two unknowns using a linear equation $\ldots \ldots \ldots \ldots$

\section{Weekly Checklist}

Complete MALL time.

Work in ALEKS and Notebook at least 3 days a week.

Complete the weekly Module and Notebook pages by the due date.

Attend Focus Group.

Actively participate in Focus Group.

Earn extra credit: Complete 10 topics by 


\section{Word problem with addition or subtraction of integers}

Learning Page Carefully read through the example on the Learning Page.

YOU TRY IT: The table gives the average high temperature for a week in January in Newfoundland.

1. How much higher was the average temperature on Sunday than on Wednesday?

\begin{tabular}{c|c} 
Day & High Temp $\left({ }^{\circ} \mathrm{C}\right)$ \\
\hline Mon & -2 \\
Tues & -6 \\
Wed & -7 \\
Thurs & 0 \\
Fri & 1 \\
Sat & 8 \\
Sun & 10
\end{tabular}

\section{Least common multiple of 2 numbers}

? Open the Instructor Added Resource which will direct you to a video to complete the following.

Write the least common multiple of the numbers given.

a. Least common multiple of and : We write $\operatorname{lcm}($ ) $=$ Explain why this is true.

b. Least common multiple of and : We write $1 \mathrm{~cm}($ ). 
Prime Factorization Method

Prime factorization of

Prime factorization of

$\operatorname{lcm}(24,18)=$

Underline the factors needed for 24 .

Circle the factors needed for 18 .

\section{List the Multiples Method}

Multiples of

Multiples of

Circle the least common multiple of 24 and 18. 
EXAMPLE: Find the LCM of 10 and 8. Listing Multiples

- List the multiples of the largest number: Multiples of 10 are 10, 20,30, 40, 50, 60, 70, 80, 90, ...

- Find the first multiple that is also divisible by the smaller number: We see 40 and 80 are common multiples of 10 AND 8.

- Thus, the smallest (least) common multiple of both 10 and 8 is 40 .

\section{Prime factorization}

- Find the prime factorization of both numbers: $8=2 \times 2 \times 2=2^{3}$ and $10=2 \times 5$.

- The LCM must include the greatest number of each prime factor: The LCM of 10 and 8 must include $2^{3}$ and $5^{1}$ as factors.

- This makes the LCM $2^{3} \cdot 5=8 \cdot 5=40$.
YOU TRY IT:

2. Find the LCM of 18 and 14

3. Find the LCM of 13 and 4

\section{Addition and subtraction of 3 fractions involving signs}

Watch Video 6: Practice with Subtraction of Real Numbers to complete the following. NOTE: This may not be the first video that pops up. Select this video from the list of videos on the left of the video box.

\section{PROCEDURE Subtracting Real Numbers}

If $a$ and $b$ are real numbers, then

Complete ONLY part a. Subtract.

a. 


\section{EXAMPLE:}

Add or subtract. $\frac{1}{8}-\frac{3}{16}-\left(-\frac{3}{4}\right)$

First we find the least common denominator.

Here the LCD is 16.

$$
\begin{aligned}
\frac{1}{8}-\frac{3}{16}-\left(-\frac{3}{4}\right) & =\frac{2}{2} \cdot \frac{1}{8}-\frac{3}{16}-\frac{4}{4} \cdot\left(-\frac{3}{4}\right) \\
& =\frac{2}{16}-\frac{3}{16}-\left(-\frac{12}{16}\right) \\
& =\frac{2-3-(-12)}{16} \\
& =\frac{2-3+12}{16}=\frac{11}{16}
\end{aligned}
$$

YOU TRY IT:

Add or subtract.

4. $\frac{3}{7}-\frac{2}{3}-\frac{2}{21}$

\section{Signed fraction division}

$D$ Watch Video 9: Dividing Real Numbers Involving Fractions to complete the following.

Simplify.

a.

EXAMPLE: Divide $-\frac{8}{9} \div \frac{3}{7}$.

We multiply by the reciprocal.

$$
\begin{aligned}
-\frac{8}{9} \div \frac{3}{7} & =-\frac{8}{9} \cdot \frac{7}{3} \\
& =\frac{-8 \cdot 7}{9 \cdot 3} \\
& =-\frac{56}{27}
\end{aligned}
$$

b.

\section{YOU TRY IT:}

5. Divide $-\frac{9}{2} \div \frac{6}{7}$. 


\section{Division involving zero}

Learning Page We will use the following facts for division involving 0.

Fact 1: 0 by any (other than ) equals

Fact 2: Any number by is

Click on the why icon to complete the following.

\section{Understanding division with 0}

Consider, for example,

To get the answer, we ask "What number by 4 gives $?$

The answer is That is, since we get that

Next, consider $0 \div 4$.

To get the answer, we ask "What number by gives 0 ?

The answer is That is, since we get that

Finally, consider $4 \div 0$

To get the answer we ask "What number multiplied by gives $?$

But there is because any number by gives 0 .

Thus is We get the same result with any number, not just 4 .

So, any number by is

YOU TRY IT: Evaluate each expression.
6. $\frac{13}{0}=$
7. $\frac{0}{13}=$ 


\section{Exponents and integers: Problem type 1}

Watch Video 10: Simplifying Expressions Involving Exponents to complete the following.

DEFINITION Definition of $b^{n}$

Suppose that $b$ is a and $n$ is a

Then,

$$
b^{n}=
$$

Simplify.

a.

c.

\section{Exponents and integers: Problem type 2}

d.

$\square$ Watch the video Exercise: Evaluating an exponential expression to complete the following. NOTE: This may not be the first video that pops up. Select this video from the list of videos on the left of the video box.

Evaluate the expression.

YOU TRY IT: Simplify.
8. $(-6)^{2}$
9. $-6^{2}$ 


\section{Exponents and signed fractions}

$\square$ If you not already done so, watch Video 10: Simplifying Expressions Involving Exponents and take notes on the previous page under the topic Exponents and integers: Problem type 1 or watch the video again for a review.

YOU TRY IT:
10. $-(-5)^{3}$
11. $\left(-\frac{1}{5}\right)^{3}$

\section{Order of operations with integers and exponents}

Learning Page We must follow the rules for order of operations. Here is that order.

1.

2.

3.

4.

YOU TRY IT: Simplify.

12. $4^{2}-(5-2)^{2} \cdot 3$

13. $\frac{1^{2}-(-3)^{2}+2}{1-2^{2}}$ 


\section{Properties of real numbers}

Learning Page Complete the following chart. For these properties, we suppose that $x, y$, and $z$ are real numbers.

\begin{tabular}{|l|l|l|}
\hline Commutative & Properties of Addition & Properties of Multiplication \\
\hline Associative & & \\
\hline Identity & & \\
\hline Inverse & Distributive Property \\
\hline \multicolumn{2}{|c|}{ and } \\
\hline \multirow{2}{*}{ Multiplication Property of Zero } \\
\hline
\end{tabular}

YOU TRY IT: State the Property of Real Numbers that is used.

14. $3+(6+x)=(3+6)+x$

16. $7 \cdot x=x \cdot 7$

15. $4 \cdot(y+3)=4 \cdot y+4 \cdot 3$ 


\section{Distributive property: Integer coefficients}

$\square$ Watch Video 5: Practice Applying the Distributive Property to complete the following.

Apply the distributive property of multiplication over addition.

1.

3.

YOU TRY IT: Simplify.

17. $-3(4 x-5)$
2.

4.

18. $(3 x+2) 7$

Using distribution with double negation and combining like terms to simplify: Multivariate

$\triangleright \quad$ Watch Video 9: Simplifying an Expression with Nested Parentheses to complete the following.

Simplify the expressions by clearing parentheses and combining like terms.

1.

2. 
YOU TRY IT: Simplify the expressions by clearing parentheses and combining like terms.
19. $-3(4 x-5 y)+2(7 x+y)$
20. $2(3 x+4 y)-(x+5 y)-3 x$

\section{Additive property of equality with signed fractions}

응 Open the e-book to complete the following.

\section{Addition and Subtraction Properties of Equality}

Let $a, b$, and $c$ represent real numbers.

Addition property of equality: If $a=b$, then

Subtraction property of equality: If $a=b$, then

EXAMPLE: Solve $x+\frac{4}{5}=\frac{2}{3}$ for $x$.

Subtract $\frac{4}{5}$ from both sides of the equation.

$$
\begin{aligned}
x+\frac{4}{5} & =\frac{2}{3} \\
x+\frac{4}{5}-\frac{4}{5} & =\frac{2}{3}-\frac{4}{5} \\
x+0 & =\frac{10}{15}-\frac{12}{15} \\
x & =\frac{10-12}{15} \\
x & =-\frac{2}{15}
\end{aligned}
$$

Check:

$$
\begin{aligned}
&-\frac{2}{15}+\frac{4}{5} \stackrel{?}{=} \frac{2}{3} \\
&-\frac{2}{15}+\frac{12}{15} \stackrel{?}{=} \frac{2}{3} \\
& \frac{10}{15} \stackrel{?}{=} \frac{2}{3} \\
& \frac{10^{2}}{15_{3}}=\frac{2}{3} \checkmark
\end{aligned}
$$

YOU TRY IT: Solve for $y$.

21. $y-\frac{3}{5}=\frac{2}{7}$

Check: 


\section{Multiplicative property of equality with signed fractions}

$\square$ Watch Video 3: Multiplication and Division Properties of Equality to complete the following. NOTE: This may not be the first video that pops up. Select this video from the list of videos on the left of the video box.

PROPERTY Multiplication and Division Properties of Equality

Let $a, b$, and $c$ represent real numbers. Then

- Multiplication property of equality: If $a=b$ then

- Division property of equality: If $a=b$, then provided

Solve.

1.

\section{Check:}

3.

Check:

\section{YOU TRY IT:}

2.

Check:

22. Solve $\frac{4}{3} y=-5$ for $y$. 


\section{Solving a two-step equation with integers}

$\square \quad$ Watch Video 4: Solving a Linear Equation Requiring Multiple Steps and complete the following.

Solve the equation.

\section{Check:}

YOU TRY IT: Solve.

23. $-3 y+4=10$

24. $\frac{x}{3}-5=2$

\section{Solving a two-step equation with signed fractions}

$\square$ Watch Video 6: Solving an Equation by First Clearing Fractions to complete the following.

Solve the equation. 
YOU TRY IT: Solve for $x$.

25. $\frac{2}{7} x+\frac{1}{14}=2$

\section{Solving a linear equation with several occurrences of the variable: Variables on both sides and distribution}

$\square \quad$ Watch Video 5: Guidelines for Solving a Linear Equation in One Variable and complete the following.

PROCEDURE Solving Linear Equations in One Variable

1. Simplify of the equation.

2. Collect all on of the equation.

3. Collect all on the of the equation.

4. Use the multiplication or division property of equality to obtain a for the variable.

5. Check the potential in the equation.

Solve the equation. 
YOU TRY IT:

26. Solve. $1-2 x-4=x-5(x-7)+4$

Watch Video 6: Solving a Linear Equation in One Variable to check your work.

\section{Translating a sentence into a multi-step equation}

ใ? Open the Instructor Added Resource which will direct you to a video to complete the following.

Translate the sentence into an equation.

a. Seven times the sum is

Equation:

b. The of a number is equal to 8 .

Equation:

c. Four is equal to 6.

Equation: 


\section{EXAMPLE:}

Twice the difference of $x$ and 3 is 18 .

- "Twice" means 2 times

- "difference of $x$ and 3 " is $(x-3)$.

- "is 18 " means $=18$

The equation is $2(x-3)=18$.

\section{EXAMPLE:}

3 is the same as 5 less than the quotient of 16 and a number $m$.

\section{YOU TRY IT:}

27. 7 more than the quotient of a number $d$ and 6 is 9 .

- "is the same as 3 " means $3=$.

- the "quotient of 16 and a number $m$ " is written $\frac{16}{m}$

- "5 less than" is $\frac{16}{m}-5$

The equation is $3=\frac{16}{m}-5$ or $\frac{16}{m}-5=3$

\section{Writing a multi-step equation for a real-world situation}

$D \quad$ Watch Video 3: An Application with Consecutive Integers to complete the following. NOTE: This may not be the first video that pops up. Select this video from the list of videos on the left of the video box.

The sum of consecutive integers is Find the integers.

Let _ be

is

is third(

The integers are 


\section{Writing a one-step expression for a real-world situation}

ใृ Open the Instructor Added Resource which will direct you to a video to complete the following.

Write the algebraic expression for each situation.

1. Treyvan ran a race. He ran 6 miles an hour, and the race took him $t$ hours to complete.

Write the expression for the question: How $?$

Expression: miles.

2. The Super Swing golf team won $\$ 5989$ playing in golf tournaments last year. The winnings were split evenly among the $p$ players.

Write the expression for the question: How $?$

Expression: dollars.

3. Emerson made 72 chocolate chip cookies and $w$ raisin cookies.

Write the expression for the question: How ?

Expression:

4. Jim received a prize of $x$ dollars from a chess tournament. The tournament cost him 50 dollars to enter.

Write the expression for the question: What were ?

Expression: dollars.

EXAMPLE:

Last week Missy spent 84 hours gaming. This week she spent $g$ hours gaming. Using $g$ write an expression for how many more hours Missy spent gaming last week than this week.

Missy spent $84-g$ more hours gaming last week than this week.

\section{YOU TRY IT:}

28. Fred watched only 2 episodes of "Game of Thrones" and George binge watched $t$ episodes. Using $t$ write an expression for how many more episodes George watched than Fred. 
Module 1

Solving a word problem with two unknowns using a linear equation

$\square$ Watch the video Introduction to Problem Solving to complete the following.

\section{Step 1}

Step 2

Step 3

Step 4

Step 5

Step 6

Let _ be

is number

number -

number $=24$

The smaller number is

The larger number is 
Notes from Focus Group: 
Module 1

Notes from Focus Group: 


\section{Module 2}

\section{Contents}

$\square$ Writing an inequality for a real-world situation $\ldots \ldots \ldots$

$\square$ Writing an inequality given a graph on the number line . . . . . . . . . . . . . . . . . . . . . . . .

Graphing a linear inequality on the number line . . . . . . . . . . . . . . . . . 47

$\square$ Graphing a compound inequality on the number line . . . . . . . . . . . . . . . 48

Set-builder and interval notation . . . . . . . . . . . . . . . . . . . . . . . . . . . . .

Evaluating a quadratic expression: Integers . . . . . . . . . . . . . . . . . . 50

Solving for a variable in terms of other variables using addition or subtraction: Advanced . . . . . 50

$\square$ Solving for a variable in terms of other variables using addition or subtraction with division . . 51

$\square$ Solving for a variable inside parentheses in terms of other variables . . . . . . . . . . . 51

Additive property of inequality with signed fractions . . . . . . . . . . . . . . . 51

$\square$ Writing a compound inequality given a graph on the number line . . . . . . . . . . . 52

Solving a two-step linear inequality: Problem type $2 \ldots \ldots$

Multiplicative property of inequality with integers . . . . . . . . . . . . . . . . 53

Solving a compound linear inequality: Graph solution, basic . . . . . . . . . . . . . . 54

$\square$ Solving a compound linear inequality: Interval notation . . . . . . . . . . . . . . 56

$\square$ Introduction to solving an absolute value equation . . . . . . . . . . . . . . . . 57

$\square$ Solving an absolute value equation: Problem Type $1 \ldots \ldots$

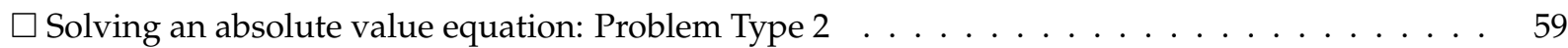

Solving inequalities with no solution or all real numbers as solutions . . . . . . . . . . 60

Translating a sentence into a multi-step inequality . . . . . . . . . . . . . . . . 61

\section{Weekly Checklist}

Complete MALL time.

Work in ALEKS and Notebook at least 3 days a week.

Complete the weekly Module and Notebook pages by the due date.

Attend Focus Group.

Actively participate in Focus Group.

Earn extra credit: Complete 10 topics by 


\section{Writing an inequality for a real-world situation}

$\square$ Watch Video 7: Translations Involving Inequalities to complete the following.

Write the expressions as mathematical inequalities.

1. The age, years.

2. A doctor ecommends that a person's daily salt intake, $s$, is at mg.

3. The total weight, in an elevator must not lb.

4. The average number of calories, $c$, that Jen takes in each day is inclusive.

\section{Writing an inequality given a graph on the number line}

Learning Page Click on the More to complete the following.

Below are four types of inequalities and their graphs.

\begin{tabular}{|l|l|}
\hline & \\
\hline$x>a$ & \\
\hline & \\
\hline$x<a$ & \\
\hline
\end{tabular}


EXAMPLE:

Write an inequality for the graph shown below.

$\begin{array}{llllllllllll}\overleftrightarrow{4} & -4 & -3 & -2 & -1 & 0 & 1 & 2 & 3 & 4 & 5\end{array}$

The shaded regions includes all numbers to the right of 2 . The closed dot at 2 indicates that 2 is also shaded. This represents all values greater than or equal to $2 \Longrightarrow x \geq 2$.
YOU TRY IT:

29. Write an inequality for the graph shown below.

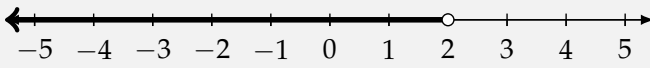

\section{Graphing a linear inequality on the number line}

응 Open the e-book to complete the following.

The set $\{x \mid x \geq 3\}$ represents all real numbers than or equal to 3.

This set can be illustrated graphically on the number line. Graph the inequality in two ways below.

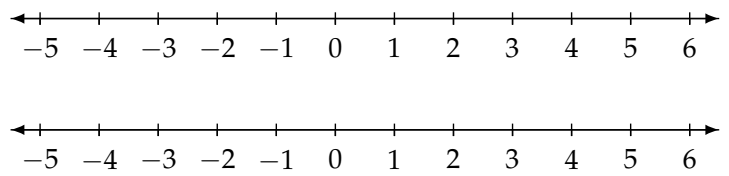

By convention, a circle $\bullet$ or a bracket [is used to indicate that an endpoint is in the set.

The set $\{x \mid x>3\}$ represents all real numbers greater than or equal to 3.

This set can be illustrated graphically on the number line. Graph the inequality in two ways below.

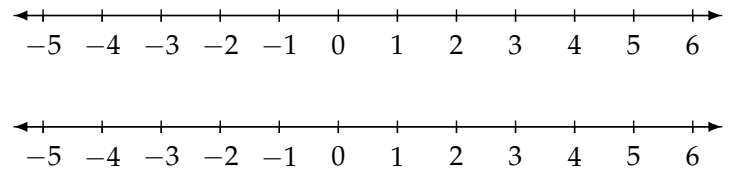

By convention, an circle $\circ$ or a (is used to indicate that an enpoint is included in the set. 
EXAMPLE: Graph $x<3$ on a number line.

- To graph $x<3$, we shade all numbers less than or equal to 3 .

- This is all numbers to the left of 3 on the number line.

- To show that 3 is not included in the graph, we use an open dot, $\circ$.

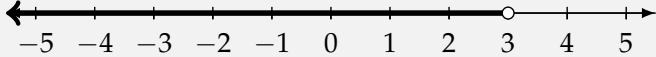

\section{YOU TRY IT:}

30. Graph $b>-1$ on a number line.

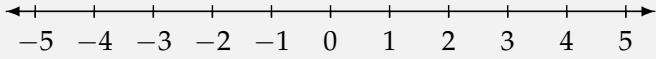

\section{Graphing a compound inequality on the number line}

Aa Open the Dictionary to complete the following.

\section{Compound Inequality}

Two inequalities joined by the word or the the word form a

\section{Solutions to compound inequalities:}

- If the two inequalities are joined by , the solution is made up of values that

$\circ x \geq 0$ and $x<3$

The solution is made up of all the numbers

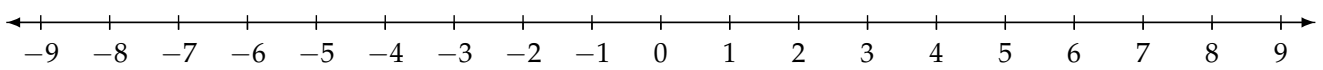

- If the two inequalities are joined by then the solution is made up of values that

○ $x \leq 0$ or $x>3$

The solution is made up of all the numbers

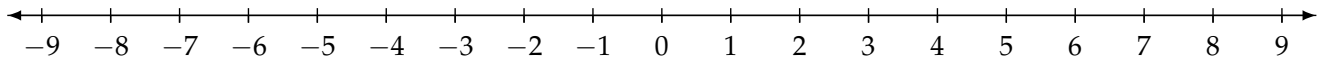




\section{EXAMPLE:}

Graph $x>1$ or $x<4$ on a number line.

The solution is made up of all the numbers.

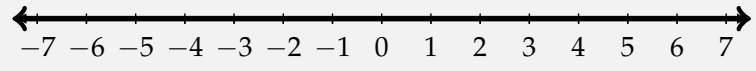

For any number $x$, at least one of these two inequalities will be true.

\section{EXAMPLE:}

Graph $x<3$ and $x>5$ on a number line.

The solution is the empty set.

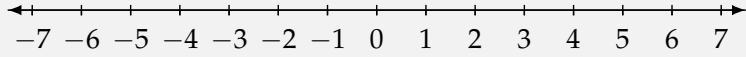

No number can be both smaller than 3 and greater than 5 .

\section{YOU TRY IT:}

31. Graph $b \leq 1$ or $b \geq 2$ on a number line.

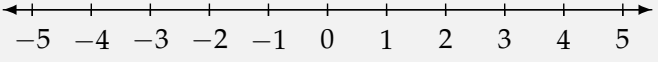

32. Graph $b \leq-2$ or $b \geq-3$ on a number line.

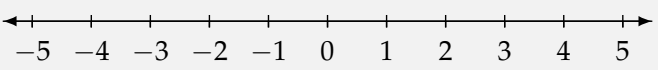

\section{Set-builder and interval notation}

Watch Video 5: Introduction to Interval Notation to complete the following.
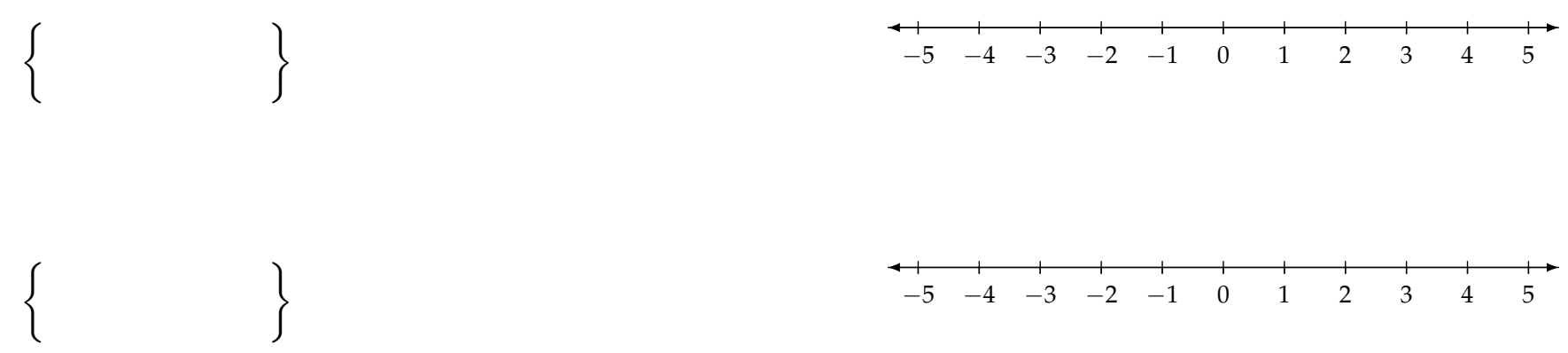

Use to exclude an endpoint.

Use to include an endpoint.

NOTE: On the number line

The and both indicate that a point IS NOT included in a set.

The and both indicate that a point IS included in a set. 


\section{Evaluating a quadratic expression: Integers}

Learning Page Carefully read through the example on the Learning Page.

EXAMPLE:

Evaluate $y^{2}-5 y+4$ when $y=-3$.

We substitute -3 for $y$, then follow the order of operations.

$$
\begin{aligned}
y^{2}-5 y+4 & =(-3)^{2}-5(-3)+4 \\
& =9-5(-3)+4 \\
& =9+15+4 \\
& =28
\end{aligned}
$$

YOU TRY IT:

33. Evaluate $b^{2}+7 b-5$ when $b=2$.

Solving for a variable in terms of other variables using addition or subtraction: Advanced

$\triangleright \quad$ Watch Video 8: Solving an Equation for a Given Variable to complete the following. NOTE: This may not be the first video that pops up. Select this video from the list of videos on the left of the video box.

Solve for $P$.

\section{YOU TRY IT:}

34. Solve for $x . A+y-x=12$ 
Solving for a variable in terms of other variables using addition or subtraction with division

$\square$ Watch Video 9: Writing an equation $A x+B y=C$ in the Form $y=m x+b$ to complete the following.

Solve for $y$.

Solving for a variable inside parentheses in terms of other variables

Learning Page Carefully read through the example on the Learning Page.

YOU TRY IT: Solve.

35. $A=7(x+4)$

\section{Additive property of inequality with signed fractions}

Open the e-book to complete the following.

\section{Addition and Subtraction Properties of Inequality}

Let $a, b$, and $c$ represent real numbers.

*Addition property of Inequality: If $a<b$, then

*Subtraction property of Inequality: If $a<b$, then

*These properties may also be stated for $a \leq b, a>b$, and $a \geq b$. 
YOU TRY IT: Solve.
36. $x+4<9$
37. $-\frac{3}{4}+x \geq \frac{1}{8}$

\section{Writing a compound inequality given a graph on the number line}

Aa Open the Dictionary and select Compound Inequality. If you have not already done so, take notes under the topic Graphing a compound inequality on the number line.

\section{EXAMPLE:}

Write a compound inequality for the graph shown below.

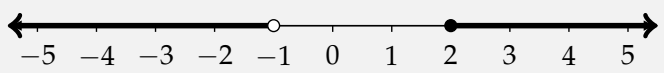

The shaded regions include all numbers less than -1 or all numbers greater than or equal to $2 \Longrightarrow x<-1$ or $x \geq 2$.

\section{YOU TRY IT:}

38. Write a compound inequality for the graph shown below.

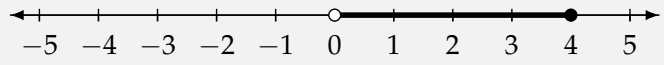

\section{Solving a two-step linear inequality: Problem type 2}

$\triangleright \quad$ If you have not already done so, watch Video 5: Multiplication and Division Properties of Inequality and take notes under the next topic Multiplicative property of inequality with integers.

EXAMPLE: Solve $15<21-2 x$.

$15-21<21-2 x-21$ isolate the variable term

$-6<-2 x$

$\frac{-6}{-2}>\frac{-2 x}{-2} \quad \begin{gathered}\text { divide both sides by }-2 \\ \text { and reverse the inequality }\end{gathered}$

$3>x$

$$
(-\infty, 3)
$$

YOU TRY IT:

39. Solve $12 \geq 6+3 y$ 


\section{Multiplicative property of inequality with integers}

Watch Video 5: Multiplication and Division Properties of Inequality to complete the following. NOTE: This may not be the first video that pops up. Select this video from the list of videos on the left of the video box.

\section{PROPERTY Multiplication and Division Properties of Inequality}

Let $a, b$, and $c$ represent real numbers.

*If $c$ is positive and $a<b$, then and

${ }^{*}$ If $c$ is negative and $a<b$, then and

*These properties may also be stated for $a \leq b, a>b$, and $a \geq b$.

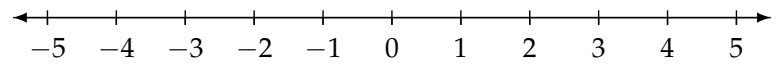

If you multiply or divide both sides of an inequality by a

you must the inequality sign.

Solve the inequality. Express the solution set in set-builder notation and in interval notation.

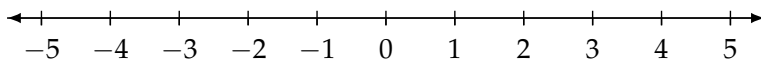

YOU TRY IT: Solve.
40. $\frac{1}{7} x<-9$
41. $-\frac{3}{4} x \geq 8$ 


\section{Solving a compound linear inequality: Graph solution, basic}

$\square$ Watch Video 7: Solving an Inequality of the Form $a<x<b$ to complete the following. NOTE: This may not be the first video that pops up. Select this video from the list of videos on the left of the video box.

\section{DEFINITION Inequalities of the form $a<x<b$.}

An inequality of the form is equivalent to the compound inequality

$$
a<x<b
$$

$a$

AND $x$

A inequality is a convenient way of writing a

Solve the inequality.

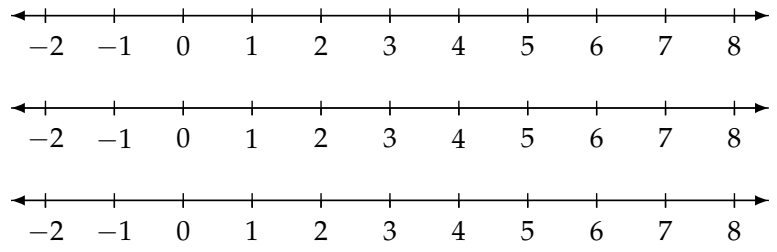

Show the "easier" way to solve this inequality:

\section{Continued on the next page.}


$\square$ Watch Video 9: Solving a Compound Inequality Joined by "Or" to complete the following. NOTE: This may not be the first video that pops up. Select this video from the list of videos on the left of the video box.

\section{PROCEDURE Solving Compound Inequalities}

- The solution to two inequalities joined by the word AND is the of their solution sets.

- The solution to two inequalities joined by the word OR is the of their solution sets.

Solve the compound inequality.

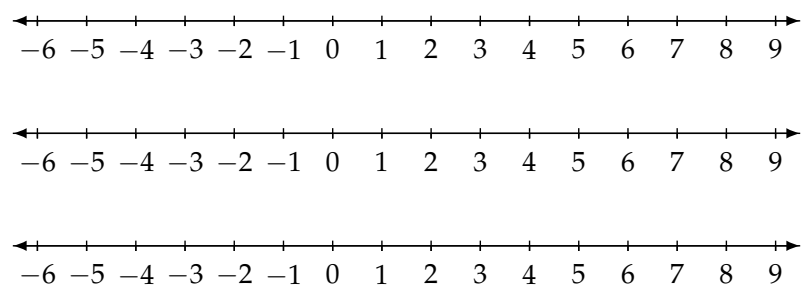

\section{YOU TRY IT:}

42. Solve $-3 x-5<4$ and $4-x \geq 6$.

43. Solve $-3 x-5 \geq 4$ or $4-x<6$. 


\section{Solving a compound linear inequality: Interval notation}

$\square$ Watch the video Mixed Exercises: Compound Inequalities to complete the following. NOTE: This may not be the first video that pops up. Select this video from the list of videos on the left of the video box.

Solve the inequality. Write the answer in interval notation.

1.

2. 


\section{Introduction to solving an absolute value equation}

$\square$ Watch Video 1: Introduction to Absolute Value Equations to complete the following.

Solve the equation.

1.

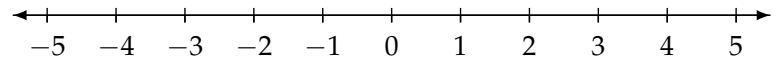

"We can interpret the absolute value of $x$ as the

on the

So geometrically, if the , then $x$

must either be at a or $"$.

\begin{tabular}{l} 
Definition \\
$|x|=\left\{\begin{array}{ll|}x & \text { if } \\
-x & \text { if }\end{array}\right.$ \\
\hline
\end{tabular}

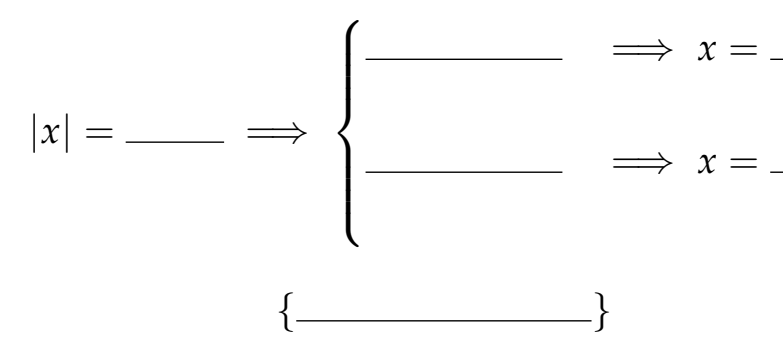

PROCEDURE Solutions to Absolute Value Equations

If $a$ is a real number, then the equation $|x|=a$ is equivalent to or

Solve the equation.

1.

2.

3. 
Module 2

\section{YOU TRY IT:}

44. Solve $|x|=3$

45. Solve $|x|=-7$

\section{Solving an absolute value equation: Problem Type 1}

$\square$ Watch the video Exercise: Solving Absolute Value Equations to complete the following.

Solve the absolute value equation.

\section{YOU TRY IT:}

46. Solve $|x|+4=11$. 


\section{Solving an absolute value equation: Problem Type 2}

$\square$ Watch Video 2: Solving an Absolute Value equation to complete the following.

\section{PROCEDURE Solving an Equation Involving One Absolute Value}

1. Isolate the

2. The equation is now in the form $|x|=a$.

- If then there is

- If then write the absolute value equation as an of equations

3. Solve the pair of equations from step 2 and write the solution set.

Solve. 
Module 2

Solving inequalities with no solution or all real numbers as solutions

ใ? Open the Instructor Added Resource which will direct you to a video to complete the following.

Solve the inequalities.

a.

b.

c. 
YOU TRY IT: Determine whether the inequality has no solution or all real numbers as solutions.

47.

$$
3(4-x)+20 \geq-3(x+7)
$$

\section{Translating a sentence into a multi-step inequality}

Learning Page Click on the first More to complete the following.

Here are some words to look for when translating phrases into algebraic expressions.

\begin{tabular}{|l|l|}
\hline Key Words & Operation \\
\hline & \\
\hline & \\
\hline & \\
\hline & \\
\hline & \\
\hline
\end{tabular}

Click on the second More to complete the following.

\begin{tabular}{|l|l|}
\hline Key Words & Symbol \\
\hline & \\
\hline & \\
\hline S & \\
\hline & \\
\hline & \\
\hline
\end{tabular}


Module 2

Notes from Focus Group: 
Notes from Focus Group: 


\section{Module 3}

\section{Contents}

$\square$ Solving an absolute value inequality: Problem Type $1 \ldots \ldots \ldots \ldots \ldots$

$\square$ Solving an absolute value inequality: Problem Type $2 \ldots \ldots \ldots$. . . . . . . . . . 66

$\square$ Solving an absolute value inequality: Problem Type $3 \ldots \ldots \ldots$. . . . . . . . . . . . 67

Writing an absolute value inequality given a graph on the number line $\ldots \ldots \ldots 68$

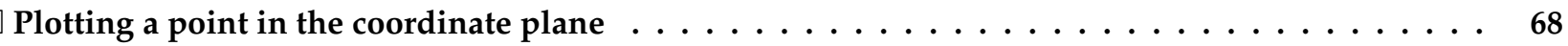

$\square$ Identifying solutions to a linear equation in two variables . . . . . . . . . . 69

$\square$ Graphing a line given its equation in slope-intercept form: Integer slope $\ldots \ldots \ldots \ldots$

$\square$ Graphing a line given its equation in slope-intercept form: Fractional slope $\ldots \ldots \ldots$. . . . . 71

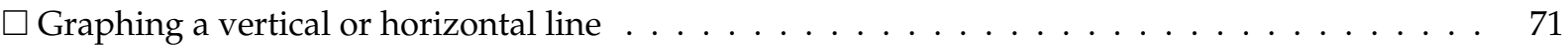

$\square$ Finding $x$ - and $y$-intercepts given the graph of a line on a grid $\ldots \ldots \ldots \ldots \ldots \ldots$

$\square$ Finding $x$ - and $y$-intercepts given the graph of a line given the equation: Basic $\ldots \ldots \ldots 74$

$\square$ Finding slope given two points on the line $\ldots \ldots \ldots \ldots \ldots \ldots \ldots \ldots$

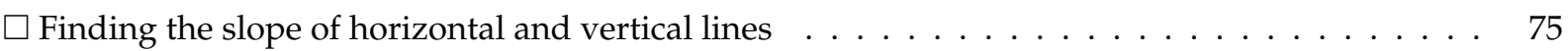

$\square$ Classifying slopes given graphs of lines $\ldots \ldots \ldots \ldots \ldots \ldots \ldots \ldots$

$\square$ Graphing a line through a given point with a given slope $\ldots \ldots \ldots \ldots \ldots$

$\square$ Graphing a line by first finding its slope and y-intercept $\ldots \ldots \ldots \ldots$

$\square$ Finding the slope and $y$-intercept of a line given its equation in the form $y=m x+b \ldots \ldots 9$

$\square$ Writing an equation in slope-intercept form given the slope and a point . . . . . . . . 79

Writing the equations of vertical and horizontal lines through a given point . . . . . . . 80

$\square$ Writing the equation of the line through two given points $\ldots \ldots \ldots \ldots 1$

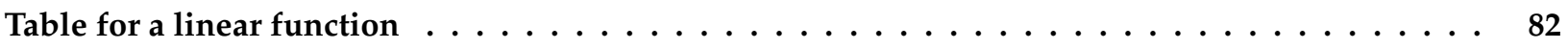

Domain and range from ordered pairs $\ldots \ldots \ldots \ldots \ldots \ldots \ldots \ldots \ldots$

\section{Weekly Checklist}

\section{Complete MALL time.}

Work in ALEKS and Notebook at least 3 days a week.

Complete the weekly Module and Notebook pages by the due date.

Attend Focus Group.

Actively participate in Focus Group.

Earn extra credit: Complete 10 topics by 


\section{Solving an absolute value inequality: Problem Type 1}

Watch Video 1: Introduction to Absolute Value Inequalities to complete the following.

Write the solution set.

1.

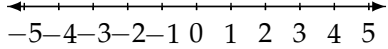

2.

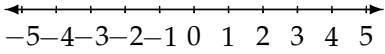

3.

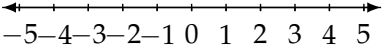

\section{DEFINITION Absolute Value Inequality}

Suppose that $a$ represents a nonnegative real number. Then,

1. The inequality $|x|<a$ is equivalent to the compound inequality:

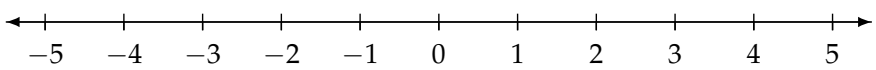

2. The inequality $|x|>a$ is equivalent to the compound inequality:

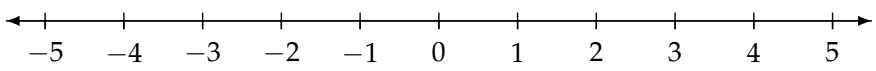

*Both cases can also be stated using the inequality symbols $\leq$ and $\geq$.

\section{YOU TRY IT:}

48. Graph the solution to $|x| \leq 4$ on the number line. 


\section{Solving an absolute value inequality: Problem Type 2}

Learning Page We start by isolating the

The absolute value of a quantity is its on the

This gives us the following rules when solving absolute value inequalities. Assume $c>0$.

- $|u|<c$ is equivalent to

$|u| \leq c$ is equivalent to

- $u>c$ is equivalent to

$u \geq c$ is equivalent to

- Let's consider an example:

This inequality is true, for example, when $u$ equals , or

This inequality is true, for example, when $u$ equals , or

In fact, this inequality is true when $u$ is

So is equivalent to

- Let's consider another example:

This inequality is true, for example, when $u$ equals , or

This inequality is true, for example, when $u$ equals or In fact, this inequality is true exactly when $u$ is

So is equivalent to 


\section{Solving an absolute value inequality: Problem Type 3}

Watch Video 2: Solving an Absolute Value Inequality to complete the following.

\section{PROCEDURE Solving Absolute Value Inequalities}

Suppose that $a$ represents a real number.

1. Isolate the

2. Write the absolute value inequality as an equivalent compound inequality.

- *The inequality $|x|<a$ is equivalent to:

- ${ }^{*}$ The inequality $|x|>a$ is equivalent to:

3. Solve the inequality from step 2 and write the solution set.

*Both cases can also be stated using the inequality symbols $\leq$ and $\geq$.

Write the solution set.

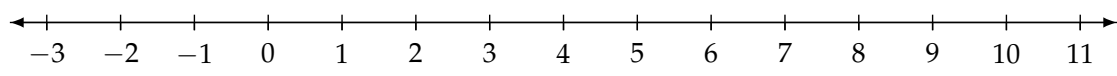

\section{EXAMPLE:}

$$
|x+3|-7 \geq-5
$$

$|x+3| \geq 2$ isolate the absolute value $x+3 \leq-2$ OR $x+3 \geq 2 \quad$ write equivalent compound inequality

$x \leq-5$ OR $x \geq-1$

$(-\infty,-5] \cup[-1, \infty)$

\section{YOU TRY IT:}

49. Graph the solution set to $4 \leq 2|x+1|-4$.

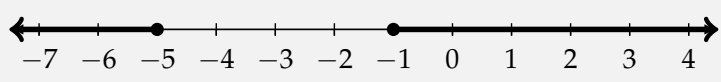




\section{Writing an absolute value inequality given a graph on the number line}

Learning Page The absolute value of a number is its on the

\section{EXAMPLE:}

Write an absolute value inequality for the graph below. Use $x$ for your variable.

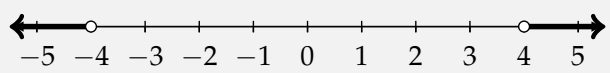

Our graph is made up of all numbers $x$ whose distance from 0 is greater than 4 . We write this as

$$
|x|>4
$$

\section{YOU TRY IT:}

50. Write an absolute value inequality for the graph below. Use $x$ for your variable.

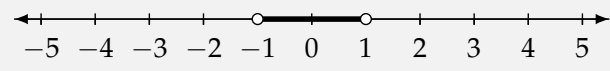

\section{Plotting a point in the coordinate plane}

\section{Watch the video Plotting Points to complete the following.}

Plot the points.

a.

b.

c.

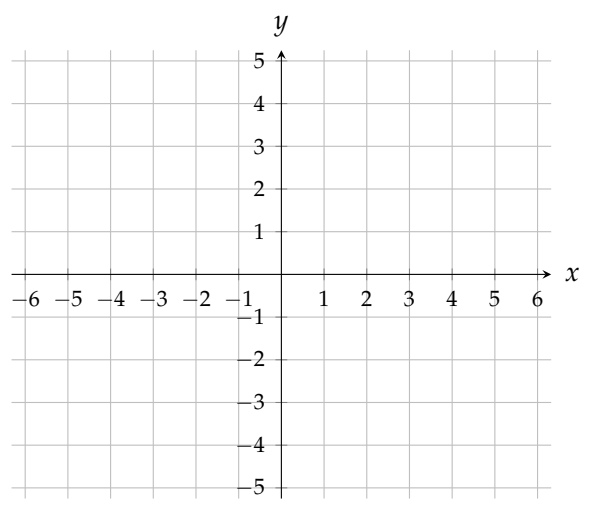

II Pause the video and try these yourself.

d.

e.

f.

Play the video and check your answers. 


\section{Identifying solutions to a linear equation in two variables}

Watch Video 4: Solutions to Linear Equations in Two Variables to complete the following.

DEFINITION Linear Equation in Two Variables

Let $A, B$, and $C$ be real numbers such that $A$ and $B$ are

A linear equation in two variables is an equation that can be written in the form:

A solution to a linear equation in two variables is an that satisfies the equation.

Determine if the ordered pair is a solution to the equation $4 x-2 y=6$.

a.

b.

c.

YOU TRY IT:

Determine if the ordered pair is a solution to the equation $3 x+5 y=-7$.
51. $(3,-2)$
52. $(-4,6)$
53. $(1,-2)$ 


\section{Graphing a line given its equation in slope-intercept form: Integer slope}

$\square \quad$ Watch Video 7: Graphing a linear equation by Using a Table of Points to complete the following.

Graph the equation

\begin{tabular}{|l|l|}
\hline$x$ & $y$ \\
\hline & \\
\hline & \\
\hline & \\
\hline
\end{tabular}

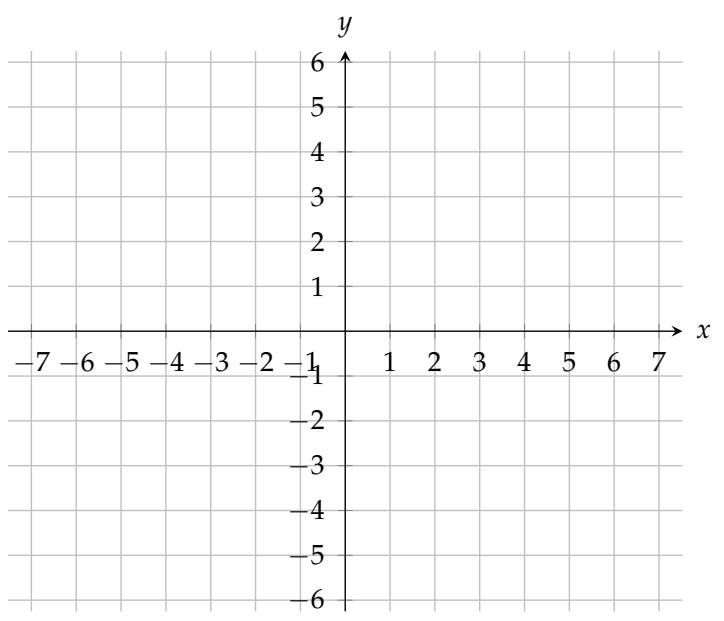

YOU TRY IT:

54. Sketch the graph of $y=2 x+4$.

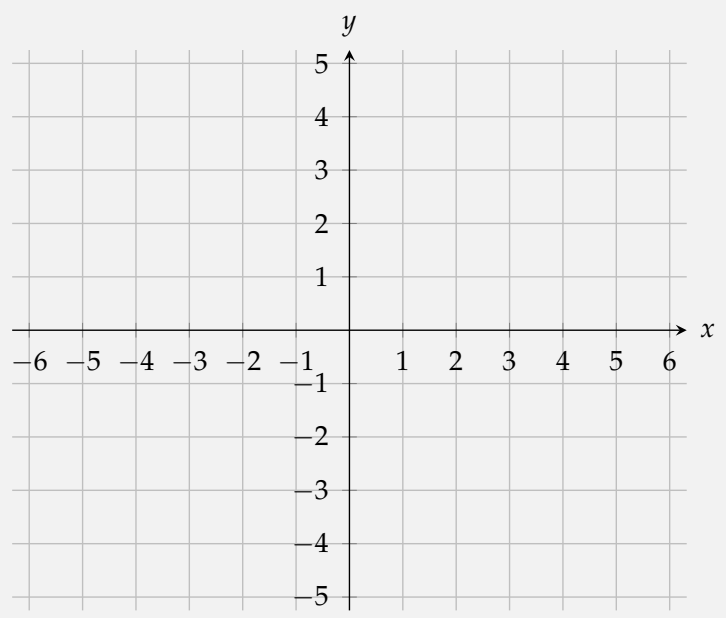




\section{Graphing a line given its equation in slope-intercept form: Fractional slope}

If you have not already done so, watch Video 7: Graphing a linear equation by Using a Table of Points and take notes under the previous topic Graphing a line given its equation in slope-intercept form: Integer slope.

YOU TRY IT: Graph the equation $y=\frac{1}{3} x-4$ by first completing the table.

55.

\begin{tabular}{|l|l|}
\hline$x$ & $y$ \\
\hline & \\
\hline & \\
\hline & \\
\hline
\end{tabular}

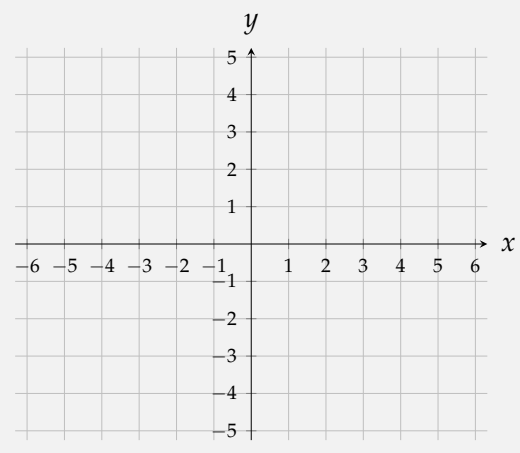

\section{Graphing a vertical or horizontal line}

Watch Video 13: Graphing Horizontal and Vertical Lines to complete the following.

Graph the equations.

1.

\begin{tabular}{|l|l|}
\hline$x$ & $y$ \\
\hline & \\
\hline & \\
\hline & \\
\hline
\end{tabular}

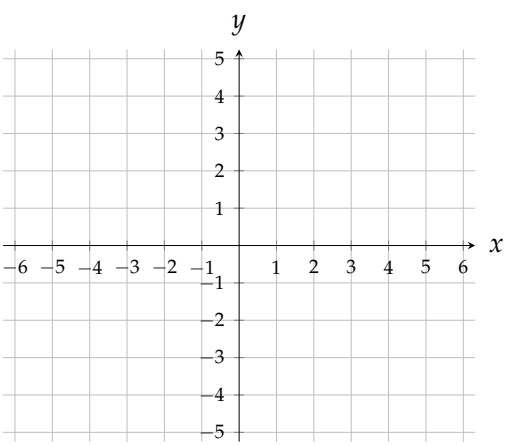

2.

\begin{tabular}{|l|l|}
\hline$x$ & $y$ \\
\hline & \\
\hline & \\
\hline & \\
\hline
\end{tabular}

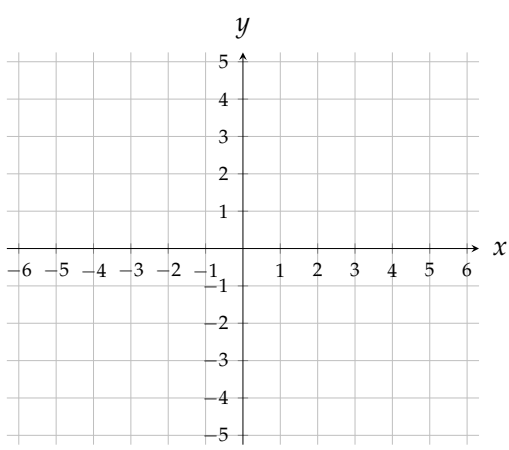




\section{YOU TRY IT:}

56. Graph $x=3$.

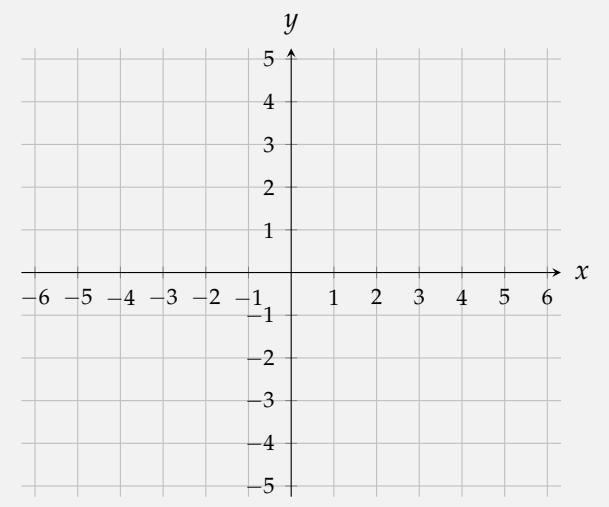

57. Graph $y=2$.

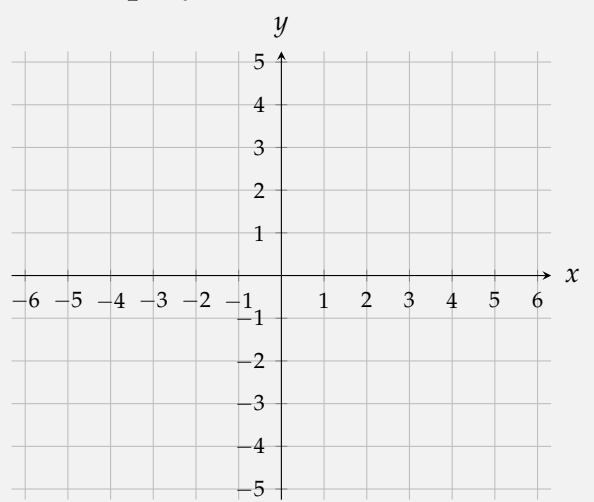

\section{Finding $x$ - and $y$-intercepts given the graph of a line on a grid}

$\square$ Watch Video 8: Introduction to $x$ and $y$-Intercepts to complete the following.

1.

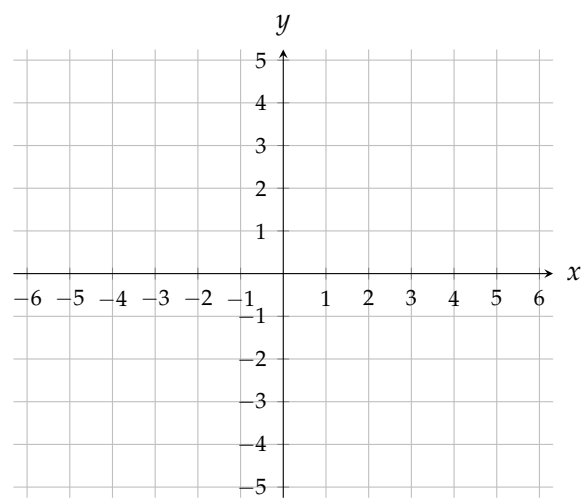

DEFINITION $x$ - and $y$-Intercepts

An $x$-intercept is a point

where a graph intersects the

A $y$-intercept is a point

where a graph intersects the

Continued on the next page 
2.

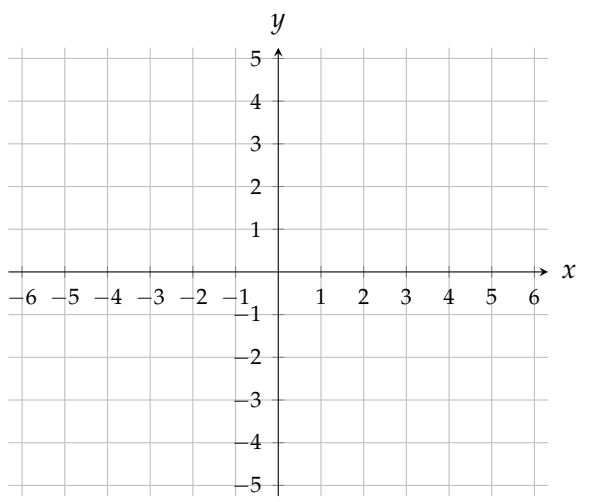

$x$-intercept:

$y$-intercept:
3.

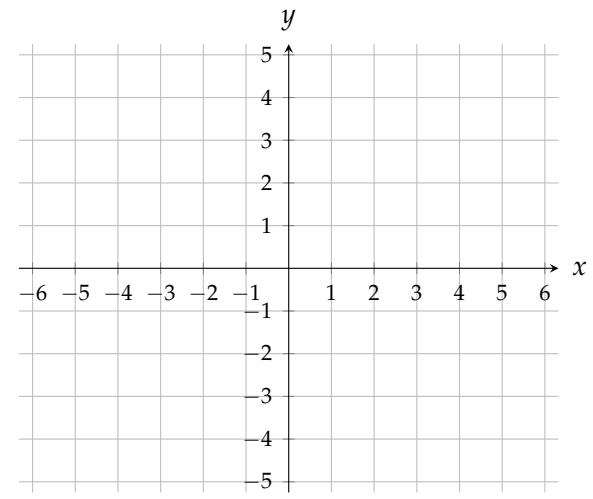

$x$-intercept:

$y$-intercept:

From the graphs in \#4 and \#5 we learn that a

- horizontal line has a

but no

- vertical line has an but no

\section{YOU TRY IT:}

58. Find the $x$ and $y$-intercepts of the graph.

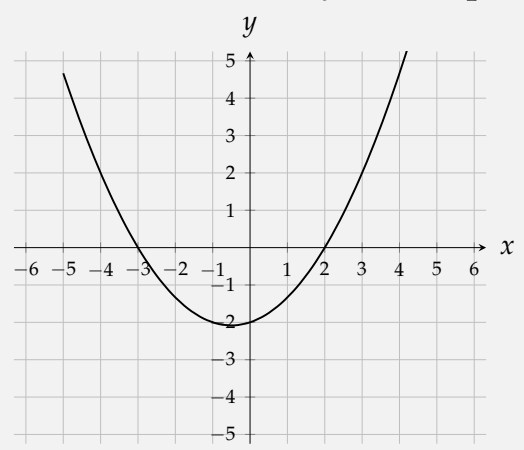




\section{Finding $x$ - and $y$-intercepts given the graph of a line given the equation: Basic}

$\square$ Watch Video 9: Finding the $x$-and $y$-Intercepts of a Line Given an Equation of the Line to complete the following.

PROCEDURE Determining the $x$ - and $y$-Intercepts from an Equation.

To find the $x$-intercept(s), substitute __ in for __ and ___ for

To find the $y$-intercept(s), in for and solve for

Determine the $x$-and $y$-intercepts and graph the equation.

\begin{tabular}{|l|l|}
\hline$x$ & $y$ \\
\hline & \\
\hline & \\
\hline & \\
\hline
\end{tabular}

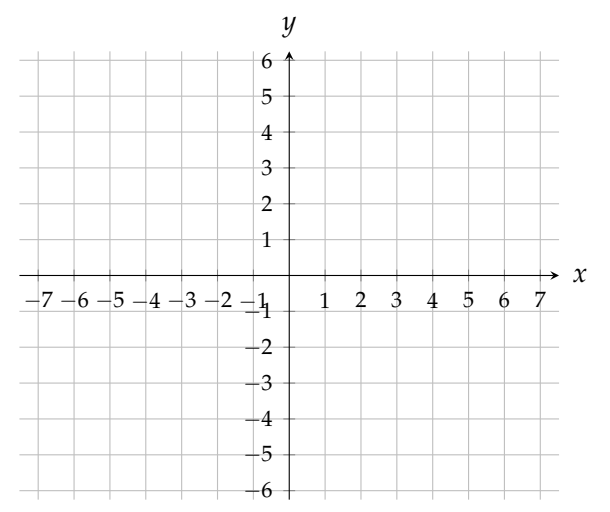

\section{Finding slope given two points on the line}

$\square$ Watch Video 3: Introduction to the Slope Formula to complete the following.

Find the slope of the line through the points and

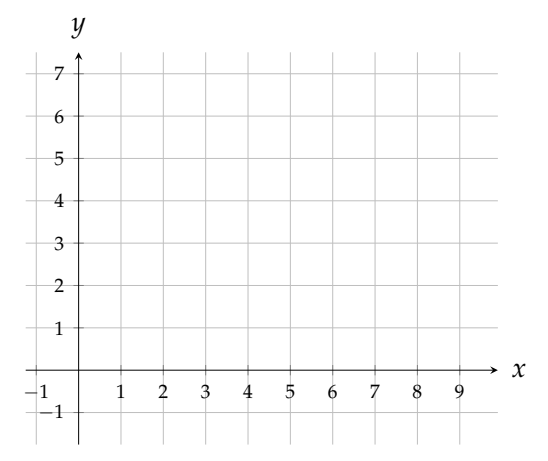

Slope formula:

Does it matter which point is labeled as point 1 and which is labeled as point 2 ? 


\section{YOU TRY IT:}

59. Find the slope of the line containing $(-1,4)$ and $(3,7)$.

\section{Finding the slope of horizontal and vertical lines}

$\square \quad$ Watch the video Video 7: Determining the Slope of a Vertical Line to complete the following.

Determine the slope of the line containing the points and

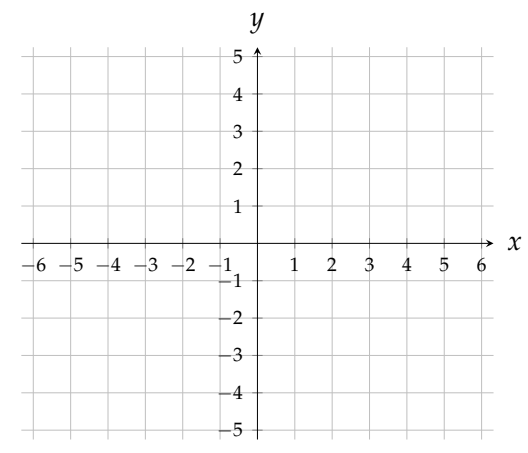

$$
m=\frac{y_{1}-y_{2}}{x_{1}-x_{2}}=
$$

Watch the video Video 8: : Determining the Slope of a Horizontal Line to complete the following. NOTE: This may not be the first video that pops up. Select this video from the list of videos on the left of the video box.

Determine the slope of the line containing the points and

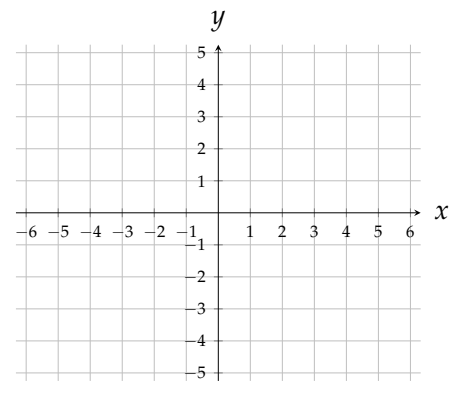

$$
m=\frac{y_{1}-y_{2}}{x_{1}-x_{2}}=
$$




\section{EXAMPLE:}

Determine the slope of the line containing the points $(-1,-3)$ and $(5,-3)$.

$$
m=\frac{y_{1}-y_{2}}{x_{1}-x_{2}}=\frac{-3-(-3)}{-1-5}=\frac{0}{-6}=0
$$

The slope is 0 .

\section{YOU TRY IT:}

60. Determine the slope of the line containing the points $(3,-2)$ and $(3,-5)$.

\section{Classifying slopes given graphs of lines}

$\square$ Watch Video 2: Orientation of a Line and the Sign of the Slope to complete the following.

\section{Show the change in $y$ and the change in $x$ on each graph.}

positive slope

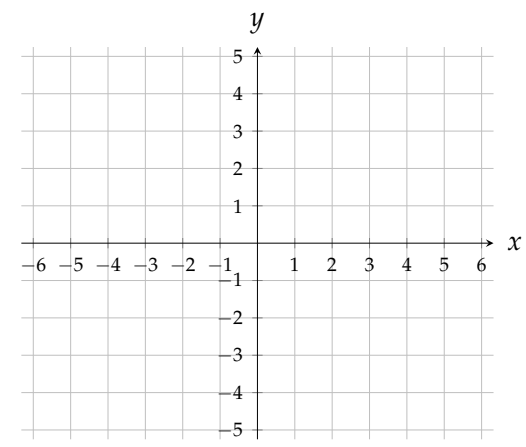

$m=\frac{\text { change in } y}{\text { change in } x}=$

zero slope

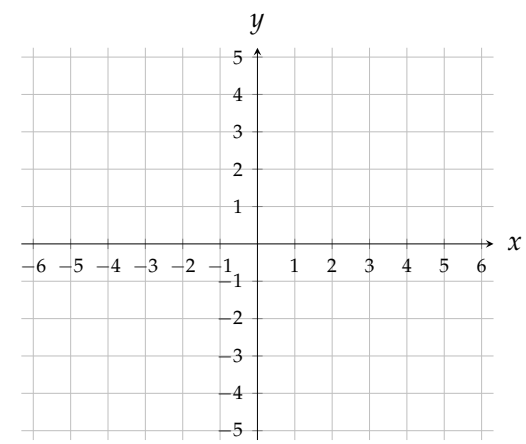

$m=\frac{\text { change in } y}{\text { change in } x}=$ negative slope

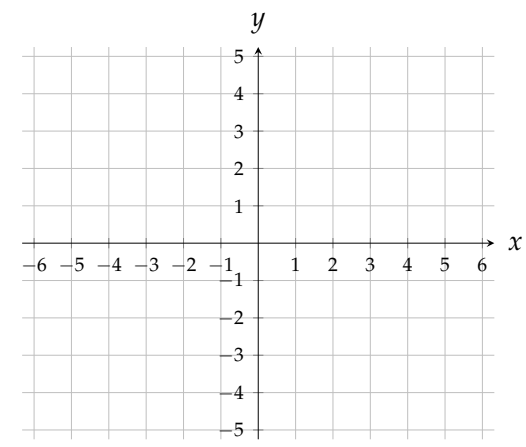

$m=\frac{\text { change in } y}{\text { change in } x}=0$

undefined slope

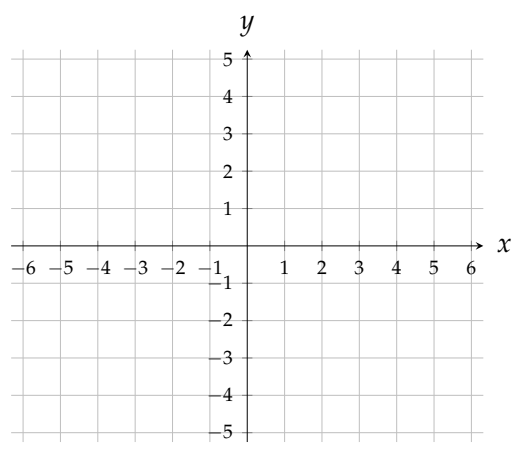

$m=\frac{\text { change in } y}{\text { change in } x}=$ 


\section{Graphing a line through a given point with a given slope}

Learning Page Graph the line with slope passing through the point

The slope is So we have the following.

$$
\text { slope }=\frac{\text { rise }}{\text { run }}=
$$

The line passes through the point

We start at and use the to find a on the line.

The is , so we to the

(This corresponds to moving units to the

\section{.)}

The , so we add to the

(This corresponds to moving unit

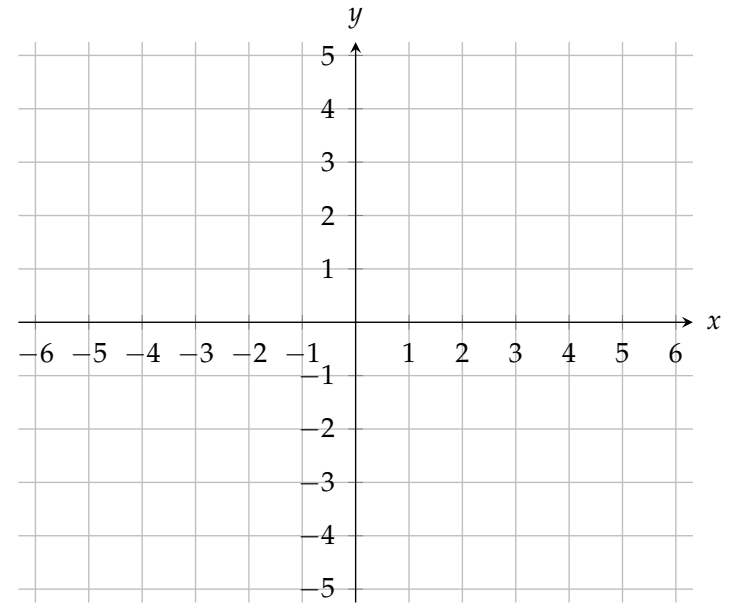
.)

We use and to graph the line.

\section{YOU TRY IT:}

61. Graph the line with slope $\frac{1}{3}$ passing through the point $(0,1)$.

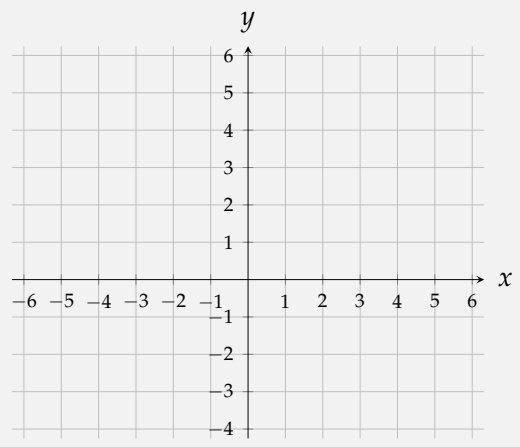




\section{Graphing a line by first finding its slope and y-intercept}

$\square \quad$ Watch the video Exercise: Graphing a Line by Using a Slope and y-Intercept to complete the following.

Write the equation in slope-intercept form (if possible). then graph each line, using the slope and y-intercept. Graph the equation

$m=$

$y$-intercept $=$

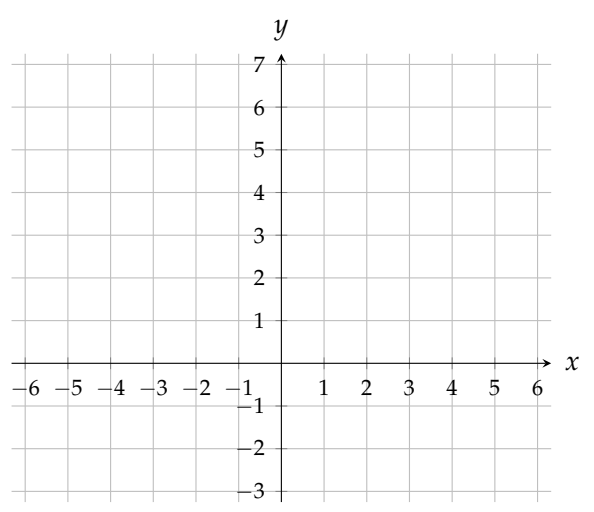

\section{YOU TRY IT:}

62. Graph the line $6 x+2 y=4$.

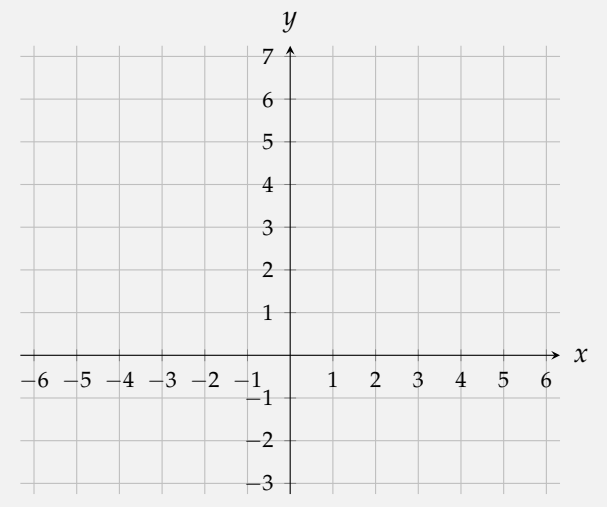

$m=$

$y$-intercept $=$ 
Finding the slope and $y$-intercept of a line given its equation in the form $y=m x+b$

Watch Video 3: Graphing a Line From its Slope and y-Intercept to complete the following.

Graph the equation by using the slope and y-intercept.

$y=$

Slope:

$y$-intercept:

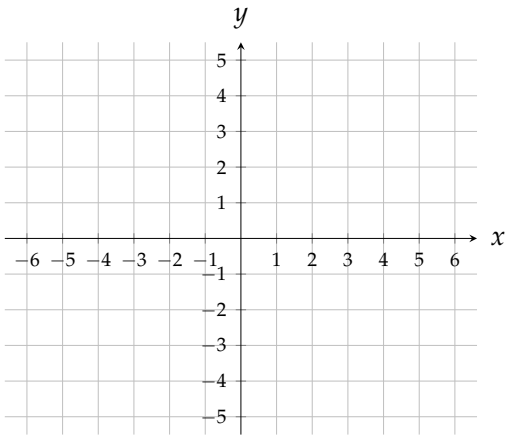

YOU TRY IT:

63. Find the slope and $y$-intercept of the line $y=-2 x+4$.

\section{Writing an equation in slope-intercept form given the slope and a point}

Watch Video 5: Using Slope-Intercept Form to Determine an Equation of a Line Given a Point on the Line and the Slope

1. Use the slope-intercept form to write an equation of a line passing through the point and having a slope of Write the answer in slope-intercept form and in standard form.

$y=m x+b$

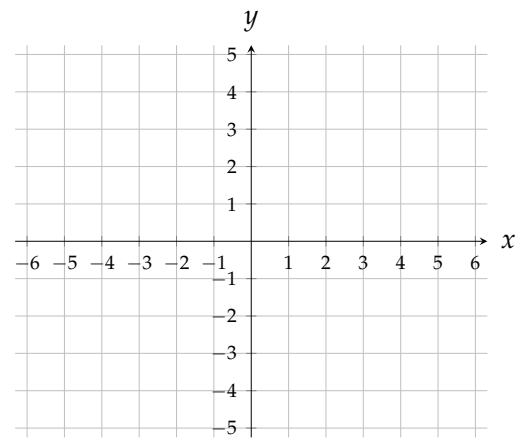


EXAMPLE: Use the slope-intercept form to write an equation of a line passing through the point $(3,8)$ and having a slope of $\frac{1}{3}$. Write the answer in slope-intercept form and standard form. Start with $y=m x+b$ and substitute $m=\frac{1}{3}$.

$$
\begin{aligned}
& y=\frac{1}{3} x+b \\
& 8=\frac{1}{3}(3)+b \quad \text { Plug in }(3,8) \text { for } x \text { and } y \\
& 8=1+b \\
& 7=b \quad \text { Solve for } b \\
& y=\frac{1}{3} x+7 \quad \text { Substitute } m \text { and } b
\end{aligned}
$$

To change the answer to standard form, subtract $\frac{1}{3} x$ to both sides to obtain: $y-\frac{1}{3} x=7$. Now multiply both sides of they equation by 3

to get: $x-3 y=-21$.

\section{YOU TRY IT:}

64. Use the slope-intercept form to write and equation of a line passing through the point $(-2,-5)$ and having a slope of 3 . Write the answer in slope-intercept form and standard form.

\section{Writing the equations of vertical and horizontal lines through a given point}

1. Determine an equation of the line passing through the point and parallel to the line defined by

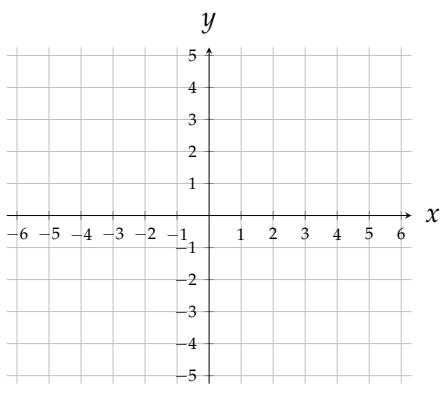

2. Determine an equation of the line passing through the point and perpendicular to the

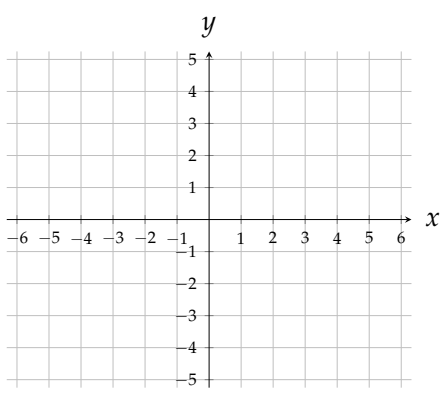


YOU TRY IT:

65. Write the equations for the horizontal and vertical lines passing through the point $(3,7)$.

\section{Writing the equation of the line through two given points}

$\square$ Watch Video 7: Writing an Equation of a Line Given Two Points on the Line to complete the following.

1. Write an equation of the line passing through the points and Write the answer in slope-intercept form.

Use the point-slope form.

2. Write an equation of the line passing through the points and Write the answer in slope-intercept form.

Use the slope-intercept form. 


\section{YOU TRY IT:}

66. Write an equation of the line passing through the points $(-2,-1)$ and $(3,-4)$.

\section{Table for a linear function}

$\square$ Watch the video Function Notation to complete the following.

$y=\ldots$ can also be written as $=2 x$.

We read $f(x)=$ as or

Given the function defined by , determine the function values.
a. $f(-2)=$
b. $f(-1)=$
c.
d.
e.

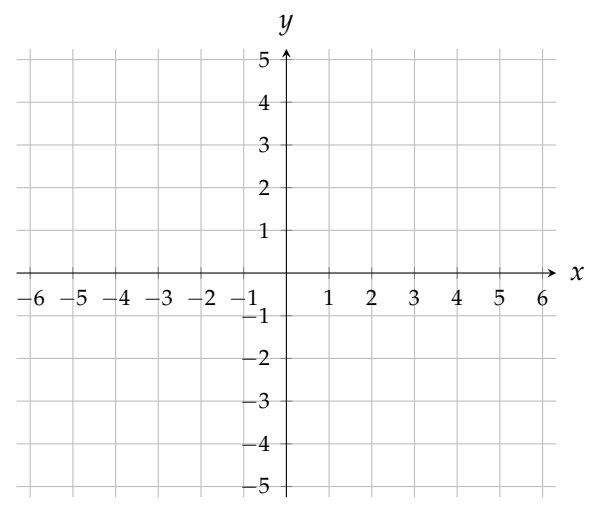

\section{YOU TRY IT:}

67. Complete the function table for

$$
f(x)=2 x+1
$$

\begin{tabular}{|c|c|}
\hline$x$ & $f(x)$ \\
\hline-2 & \\
\hline-1 & \\
\hline 0 & \\
\hline 1 & \\
\hline 2 & \\
\hline
\end{tabular}




\section{Domain and range from ordered pairs}

Watch Video 1: Introduction to Relations, Domain, and Range to complete the following.

DEFINITION

A relation in $x$ and $y$ is a

of the form $(x, y)$.

- The set of

is called the

of the relation.

- The set of is called the of the relation.

1. The table shows a relation between the number of minutes played and the number of points scored for a college basketball player.

(a) Write the relation given in the table as a set of ordered pairs.

\begin{tabular}{|c|c|}
\hline $\begin{array}{c}\text { Minutes played } \\
x\end{array}$ & $\begin{array}{c}\text { Points scored } \\
f(x)\end{array}$ \\
\hline & 18 \\
\hline 24 & \\
\hline 30 & \\
\hline & 26 \\
\hline 30 & 22 \\
\hline
\end{tabular}

(b) Determine the domain and range of the relation.

Domain:

Range:

\section{YOU TRY IT:}

68. Find the domain and range of the relation $S=\{(2,3),(-5,1),(0,3),(5,-4)\}$. 
Module 3

Notes from Focus Group: 
Notes from Focus Group: 


\section{Module 4-Review}

To help you review for your upcoming exam, this module contains all of the topics from the modules since the last exam. Topics that you have already mastered will not appear in your carousel.

Complete this module before you take the ALEKS exam.

Each exam has two parts.

- The ALEKS exam (100 pts)

- The ALEKS exam must be taken in the MALL.

- The ALEKS exam is a Comprehensive Knowledge Check.

- Your score is the number of topics you have mastered out of the number of topics you should have mastered by this point.

- If you lose topics on your ALEKS exam, your Review Module completion grade will not change.

- Your scratch work for the ALEKS exam must be numbered and turned in through Blackboard.

- The Written exam (25 pts)

- Take your written exam in class the day of your focus group.

- To study for the written exam:

- Rework your old Focus Group assignments.

- Rework any topics in ALEKS you may have lost on the ALEKS exam.

\begin{tabular}{|l|l|}
\hline & Score \\
\hline ALEKS Exam & \\
\hline Written Exam & \\
\hline
\end{tabular}




\section{Module 5}

\section{Contents}

$\square$ Square root of a perfect square with signs $\ldots \ldots \ldots \ldots \ldots \ldots$

$\square$ Domain and range from the graph of a continuous function $\ldots \ldots \ldots \ldots$

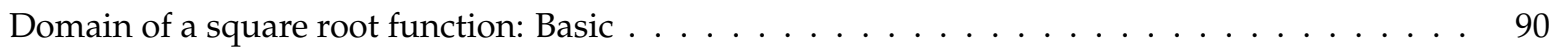

$\square$ Identifying functions from relations $\ldots \ldots \ldots \ldots \ldots \ldots \ldots \ldots \ldots$

Evaluating functions: Linear and quadratic or cubic $\ldots \ldots \ldots \ldots \ldots \ldots$

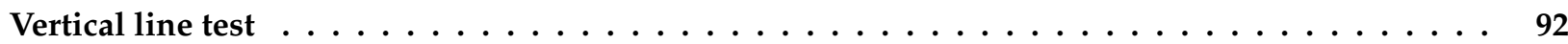

$\square$ Variable expressions as inputs of functions: Problem type $1 \ldots \ldots \ldots \ldots$

Finding outputs of a one-step function that models a real-world situation: Function notation . . 93

Finding inputs and outputs of a function from its graph $\ldots \ldots \ldots \ldots \ldots$

Identifying solutions to a system of linear equations $\ldots \ldots \ldots \ldots \ldots \ldots$

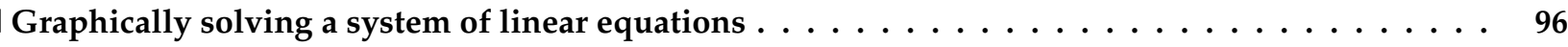

Solving a system of linear equations using substitution $\ldots \ldots \ldots \ldots \ldots \ldots$

Solving a system of linear equations using elimination with addition . . . . . . . . . . . 99

$\square$ Solving a system of linear equations using elimination with multiplication and addition $\ldots . .100$

Solving a word problem involving a sum and another basic relationship using a system of linear

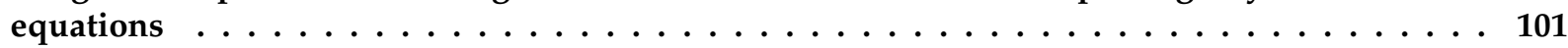

$\square$ Solving a word problem using a system of linear equations of the form $A x+B y=C \ldots 102$

$\square$ Solving a value mixture problem using a system of linear equations . . . . . . . . . . . . 103

$\square$ Solving a distance, rate, time problem using a system of linear equations . . . . . . . . . . 104

Graphing a function of the form $f(x)=a x^{2} \ldots \ldots \ldots \ldots \ldots \ldots \ldots$

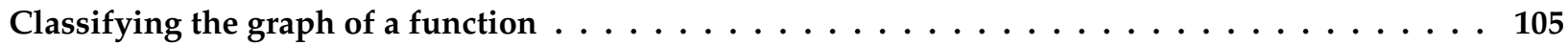

Interpreting the graphs of two functions $\ldots \ldots \ldots \ldots \ldots \ldots \ldots$

\section{Weekly Checklist}

Complete MALL time.

Work in ALEKS and Notebook at least 3 days a week.

Complete the weekly Module and Notebook pages by the due date.

Attend Focus Group.

Actively participate in Focus Group.

Earn extra credit: Complete 10 topics by 


\section{Square root of a perfect square with signs}

$\square$ Watch Video 2: Defining Square Roots Using Radical Notation to complete the following.

Simplify the expressions.

1.

4.

5.

2.

If $a$ is a positive real number, then

- $\sqrt{a}$ is the

3.

square root of $a$ (also called the

)

- $-\sqrt{a}$ is the (or opposite) square root of $a$ (also called the

- $\sqrt{0}=$

Note: In the expression $\sqrt{a}$, the symbol, $\sqrt{ }$ is called a . The value of $a$ is called the 


\section{Domain and range from the graph of a continuous function}

Watch the video Determining Domain and Range of a Relation Containing an Infinite Number of Points to complete the following.

Determine the domain and range of each relation.

a. Sketch the graph from the video.

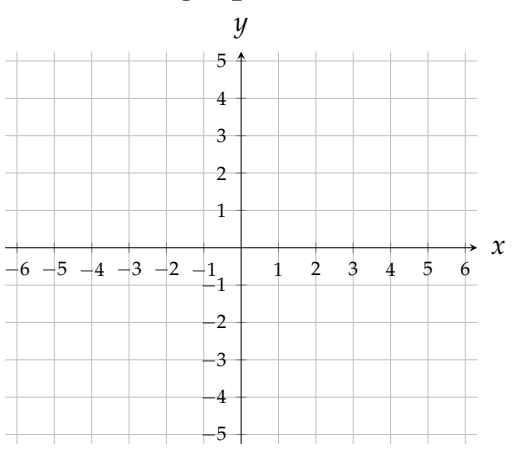

Domain:

Range: b. Sketch the graph from the video.

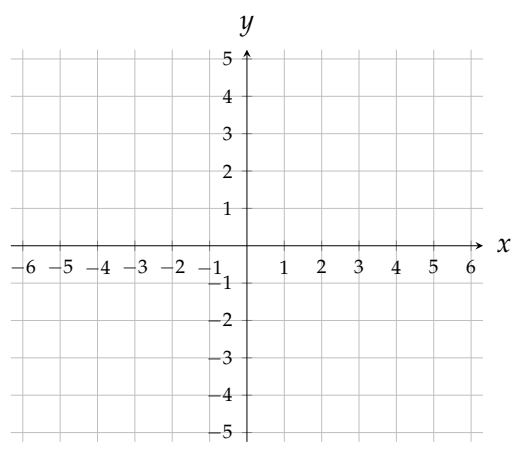

Domain:

Range:

\section{YOU TRY IT:}

69. Find the domain and range.

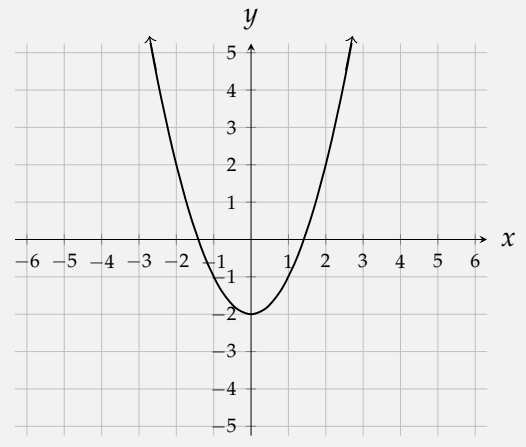




\section{Domain of a square root function: Basic}

Open the e-book to complete Example 6 Part c.

Write the domain in interval notation.

$k(t)=$

Solution:

The function defined by will not be a number when the is The domain is the set of all that

make the radicand $\geq 0$

$t \geq$

Interval notation:

EXAMPLE:

Find the domain of the function.

$$
h(x)=\sqrt{x}+100
$$

We need all values of $x$ that make the radical, $\sqrt{x}$, a real number.

The $\sqrt{x}$ will be a real number whenever, $x \geq 0$.

Therefore the domain of the function $h(x)$ is the interval $[0, \infty)$.
YOU TRY IT: Find the domain of the function.

70. $g(x)=\sqrt{x+100}$ 


\section{Identifying functions from relations}

Watch Video 1: Definition of a Function to complete the following.

\section{DEFINITION}

Given a relation in $x$ and $y$, we say that $y$ is a function of $x$ if for each element $x$ in the there is corresponding $y$ value in the

For each relation, determine if the relation defines $y$ as a function of $x$.

a. \{<smiles>[3H]I</smiles>

b. \{ \}

\section{YOU TRY IT:}

For each relation, determine whether or not it is a function.

72. $\{(2,3),(-5,1),(0,3),(5,-4)\}$.

73. $\{(1,-2),(-7,3),(1,5),(0,8)\}$.

\section{Evaluating functions: Linear and quadratic or cubic}

Watch the video Exercise: Evaluating a function to complete the following. NOTE: This may not be the first video that pops up. Select this video from the list of videos on the left of the video box.

Consider the functions defined by $f(x)=$ $g(x)=$ and Find the following. 
EXAMPLE: Given $h(x)=4-5 x^{3}$, find $h(-2)$.

Substitute -2 in for $x$ in the function $h(x)$.

$$
\begin{aligned}
h(-2) & =4-5(-2)^{3} \\
& =4-5(-8) \\
& =4+40=44
\end{aligned}
$$

Therefore, $h(-2)=44$

\section{YOU TRY IT:}

74. Given $f(x)=-2 x^{2}+3 x-4$, find $f(-3)$.

\section{Vertical line test}

$\square \quad$ Watch Video 3: Vertical Line Test to complete the following.

Determine if the relation is a function.

a.

Yes or No?

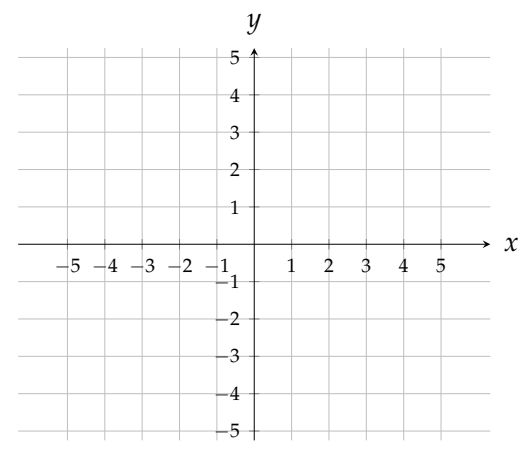

\section{PROCEDURE Vertical Line Test}

Given a relation in $x$ and $y$, the graph defines if no vertical line intersects the graph in

b.

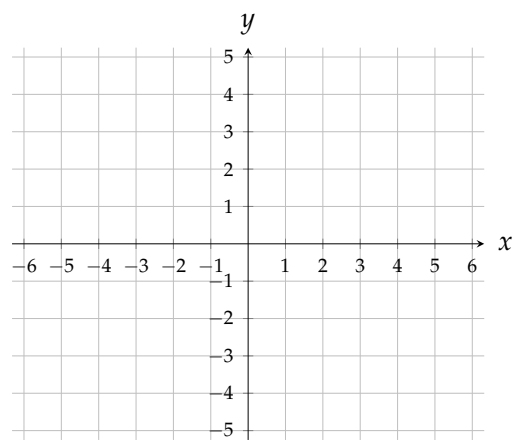

Yes or No? c.

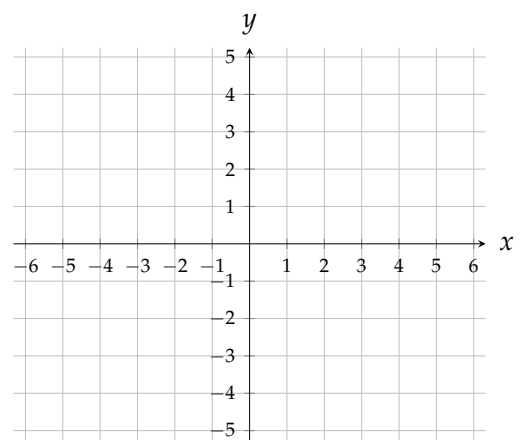

Yes or No? 


\section{Variable expressions as inputs of functions: Problem type 1}

Watch Video 5: Evaluating Functions to complete the following.

Evaluate the functions for the given values of $x$.

$$
g(x)=
$$

a. $g(t)=$

b. $h(x+3)=$

c. $g(-a)=$

d. $h(-5)=$

\section{Finding outputs of a one-step function that models a real-world situation: Function notation}

Learning Page Carefully read through the example on the Learning Page.

EXAMPLE: A one-day admission ticket to Valleyfair amusement park is $\$ 37$. The cost, $C$ (in dollars), of admission for a group of $n$ people is given by the function

$$
C(n)=37 n \text {. }
$$

What is the cost of admission for a group of 5 people?

We need to find the cost of admission $C(n)$, where the number of people, $n$, is 5 .

We let $n=5$ and write $C(5)=37 \cdot 5=\$ 185$.

The cost of admission to Valleyfair for a group of 5 people is $\$ 185$

\section{YOU TRY IT:}

Lettie and 6 of her friends are sharing the cost of pizza Rhombus Guys Pizza. If $p$ is the total cost of pizza, the amount to be paid by each person, $A(p)$ (in dollars) is given by the function

$$
A(p)=\frac{p}{7}
$$

If the total cost of the pizza is $\$ 80$ how much does each person owe? 


\section{Finding inputs and outputs of a function from its graph}

$\square$ Watch Video 6: Estimating Function Values from a Graph to complete the following.

1. Find $f(-2)$.

II Pause the video and try these yourself.

2. Find $f(1)$.

3. Find $f(5)$.

$\square$ Play the video to check your answers and complete the following.

4. For what values of $x$ is $?$

II Pause the video and try this yourself.

5. For what values of $x$ is $?$

$\square \quad$ Play the video to check your answers and complete the following.

6. Write the domain of $f$.

7. Write the range of $f$.
Sketch the graph of $y=f(x)$ from the video. 


\section{EXAMPLE:}

The graph of $f(x)$ is given below.

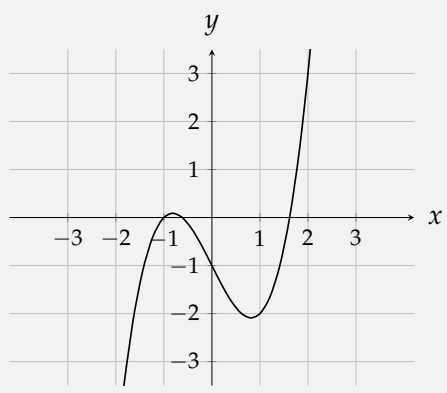

a. Use the graph to find $f(2)$.

We see the point $(2,3)$ on the graph, so $f(2)=3$.

b. Use the graph to find one value of $x$ for which $f(x)=-1$.

From the graph we see that $f(0)=-1$ so $x=0$. There are two other values of $x$ where $f(x)=-1$.
YOU TRY IT:

The graph of $g(x)$ is given below.

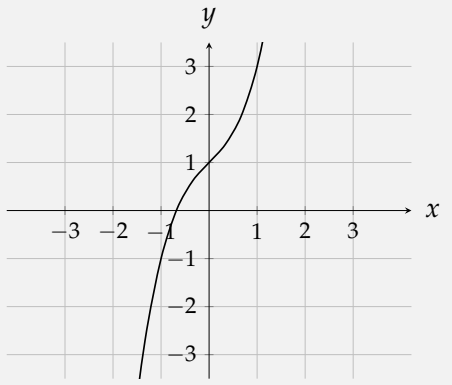

75. Use the graph to find $g(1)$.

76. Use the graph to find one value of of $x$ for which $g(x)=1$.

\section{Identifying solutions to a system of linear equations}

Watch Video 1: Determining if an Ordered Pair is a Solution to a System of Linear Equations to complete the following.

Determine if the given ordered pair is a solution to the system.
II Pause the video and try this yourself.

b. $(-2,6)$

a. $(3,1)$

Play the video to check your answer. 


\section{EXAMPLE:}

Determine if $(3,7)$ is a solution to the system

$$
\begin{aligned}
3 x-y & =2 \\
y & =x+2
\end{aligned}
$$

Equation 1:

Equation 2:

$$
\begin{array}{rlrl}
3(3)-7 & \stackrel{?}{=} 2 & 7 \stackrel{?}{=} 3+2 \\
9-7 & =2 \text { TRUE } & 7 & =5 \text { FALSE }
\end{array}
$$

Because the ordered pair $(3,7)$ does not satisfy both equations, the ordered pair is NOT a solution to the system.

\section{YOU TRY IT:}

77. Determine if $(2,4)$ is a solution to the system

$$
\begin{aligned}
3 x-y & =2 \\
y & =x+2
\end{aligned}
$$

\section{Graphically solving a system of linear equations}

$\square$ Watch Video 2: Solving a System of Linear Equations by Using the Graphing Method to complete the following.

Graph the system of equations and determine the solution set.

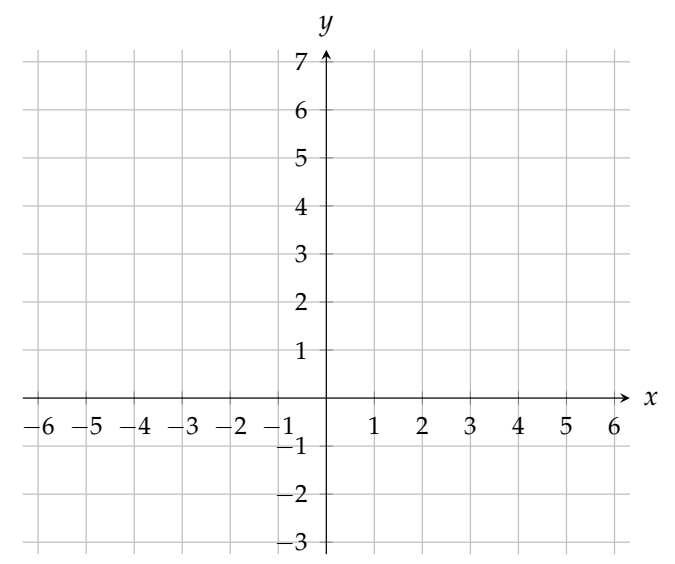


EXAMPLE: Graph the system of equations and determine the solution set.

$$
\begin{aligned}
x+2 y & =8 \\
y & =x+1
\end{aligned}
$$

Graph each equation and determine the point(s) of intersection.

Equation 1: Slope-intercept form: $y=-\frac{1}{2} x+4$

Equation 2: Slope-intercept form: $y=x+1$

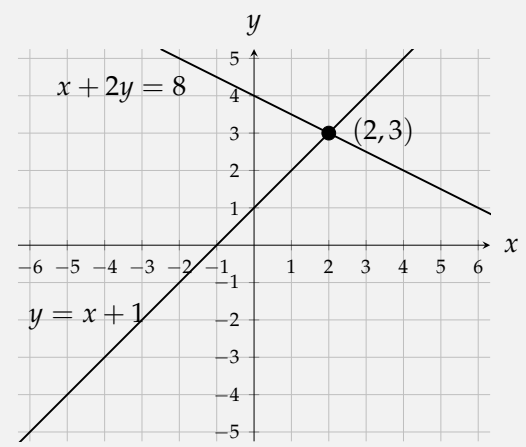

The solution set is $(2,3)$.
YOU TRY IT:

78. Graph the system of equations and determine the solution set.

$$
\begin{aligned}
& x-3 y=9 \\
& 2 x-y=-2
\end{aligned}
$$

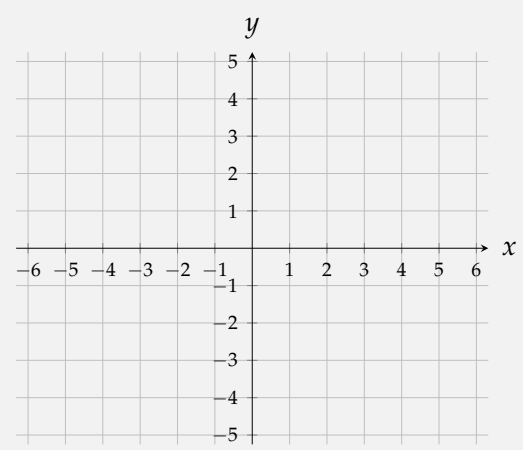

The solution set is

\section{Solving a system of linear equations using substitution}

$\square \quad$ Watch Video 1: Solving a System of Equations by Using the Substitution Method to complete the following.

Solve the system by using the substitution method.

Step 1: Isolate one of the from one of the equations.

Step 2: Substitute the found in step 1 into the other equation.

Step 3: Solve the resulting

Step 4: Substitute the of the variable found in step 3 into one of the other equations. Then solve for the remaining variable.

Step 5: Check the solution in original equations. 


\section{EXAMPLE:}

Solve the system by using the substitution method.

$$
\begin{array}{r}
-10 x+2 y=0 \\
-3 x+y=2
\end{array}
$$

Step 1: The $y$ - variable in the second equation is the easiest variable to isolate because its coefficient is 1 .

$$
\begin{aligned}
-10 x+2 y & =0 \\
-3 x+y & =2 \Rightarrow y=3 x+2
\end{aligned}
$$

Step 2: Substitute the quantity $3 x+2$ for $y$ in the other equation.

$$
-10 x+2(3 x+2)=0
$$

Step 3: Solve for $x$.

$$
\begin{aligned}
-10 x+2(3 x+2) & =0 & & \text { Simplify } \\
-4 x & =-4 & & \text { Divide by }-2 \\
x & =1 & &
\end{aligned}
$$

Step 4: Substitute the known value for $x$ into one of the original equations to solve for $y$.

$$
\begin{aligned}
-3 x+y & =2 \\
-3(1)+y & =2 \\
y & =5
\end{aligned}
$$

Step 5: Check the ordered pair $(1,5)$ in both original equations.

$$
\begin{aligned}
& -10(1)+2(5)=0 \\
& -10+10=0 \\
& 0=0
\end{aligned}
$$

$$
\begin{gathered}
-3(1)+(5)=2 \\
-3+5=2 \\
-2=2
\end{gathered}
$$

The ordered pair $(1,5)$ checks in both equations.

Therefore, the solution to the system is $\{(1,5)\}$

\section{YOU TRY IT:}

79. Solve the system by using the substitution method.

$$
\begin{array}{r}
4 x+5 y=-3 \\
x-2 y=-4
\end{array}
$$




\section{Solving a system of linear equations using elimination with addition}

Watch Video 1: Solving a System of Linear Equations by Using the Addition Method to complete the following. NOTE: This may not be the first video that pops up. Select this video from the list of videos on the left of the video box.

Solve the system by using the addition method.

Step 1: Write both equations in form $A x+B y=C$.

Step 2: Clear or decimals (optional).

Step 3: Multiply one or both equations by nonzero to create opposite coefficients for one of the variables.

Step 4: Add the equations from

Step 5: Solve for the variable.

Step 6: Substitute the value into one of the equations.

Step 7: Check the ordered pair in original equations. 


\section{YOU TRY IT:}

80. Solve the system of equations using elimination.

$$
\begin{aligned}
-2 x+5 y & =14 \\
7 x+6 y & =-2
\end{aligned}
$$

\section{Solving a system of linear equations using elimination with multiplication and ad-} dition

$D$ Watch Video 2: Solving a System of Equations by Using the Addition Method to complete the following. NOTE: This may not be the first video that pops up. Select this video from the list of videos on the left of the video box.

Solve the system by using the addition method. 


\section{Solving a word problem involving a sum and another basic relationship using a system of linear equations}

$\square$ Watch the video Applications of Systems of Linear Equations Involving Geometry to complete the following.

In a right triangle, one acute angle measures If the sum of the measures of the two acute angles must equal $90^{\circ}$, find the measures of the acute angles.

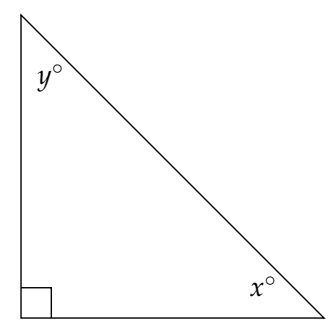

\section{YOU TRY IT:}

81. An isosceles triangle has two angles of the same measure. If the angle represented by $y$ measures $3^{\circ}$ less than the angle $x$, find the measures of all angles of the triangle.

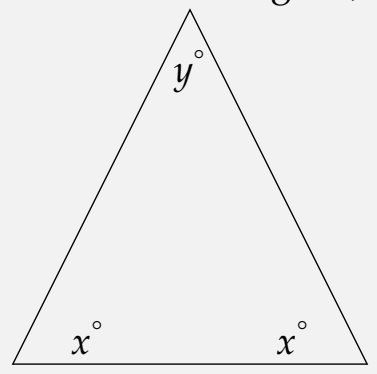


Module 5

Solving a word problem using a system of linear equations of the form $A x+B y=C$

$\square$ Watch Video 1: An Application of Systems of Linear Equations (Popcorn and Drink Sales) to complete the following.

At a movie theater, Maria bought large popcorns and drinks for Annie bought large popcorns and drinks for Determine the cost of

Let represent

Let represent

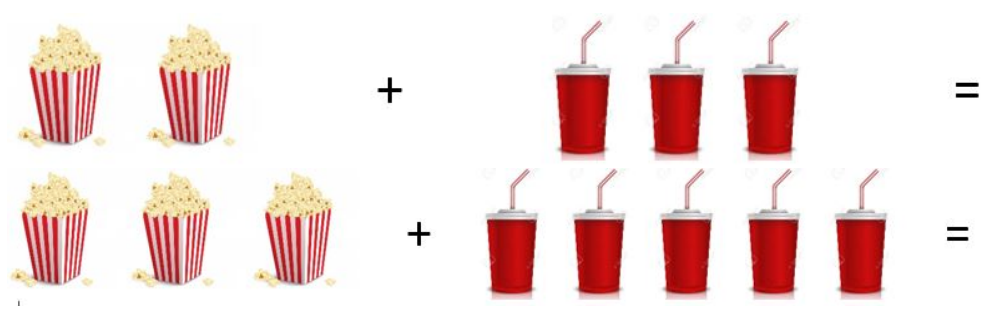




\section{YOU TRY IT:}

82. Tim and Traci bought school supplies. Tim spent $\$ 10.65$ on 4 notepads and 5 markers. Traci spent $\$ 7.50$ on 3 notepads and 3 markers. What is the cost of 1 notepad and what is the cost of 1 marker?

\section{Solving a value mixture problem using a system of linear equations}

$\square$ Watch the video Applications of Systems of Linear Equations: Mixed Exercises to complete the following.

A basketball player scored points by shooting two-point and three-point baskets. If she made a total of baskets, how many of each type did she make?

$x=$

$y=$ 


\section{Solving a distance, rate, time problem using a system of linear equations}

$\square$ Watch Video 5: Solving an Application Using Uniform Motion with a System of Linear Equations to complete the following.

Suppose a plane leaves Fargo, North Dakota headed for Orlando, FL, miles away. Going to

Orlando, the plane encounters a "tailwind" and get to Orlando in hours. Returning to Fargo, the samewind is now an opposing "headwind" and the plane takes hours to return.

Find the speed of the airplane in still air and the speed of the wind.

Let__ represent the

Let __ represent the

\begin{tabular}{|c|c|c|c|}
\hline & Distance & Rate & Time \\
\hline With a tailwind & & & \\
\hline Against a tailwind & & & \\
\hline
\end{tabular}


Graphing a function of the form $f(x)=a x^{2}$

$\square$ Watch Video 2: Graphing a function by Making a Table of Points to complete the following.

Graph the function

\begin{tabular}{|l|l|}
\hline$x$ & $f(x)$ \\
\hline & \\
\hline & \\
\hline & \\
\hline & \\
\hline & \\
\hline & \\
\hline & \\
\hline
\end{tabular}

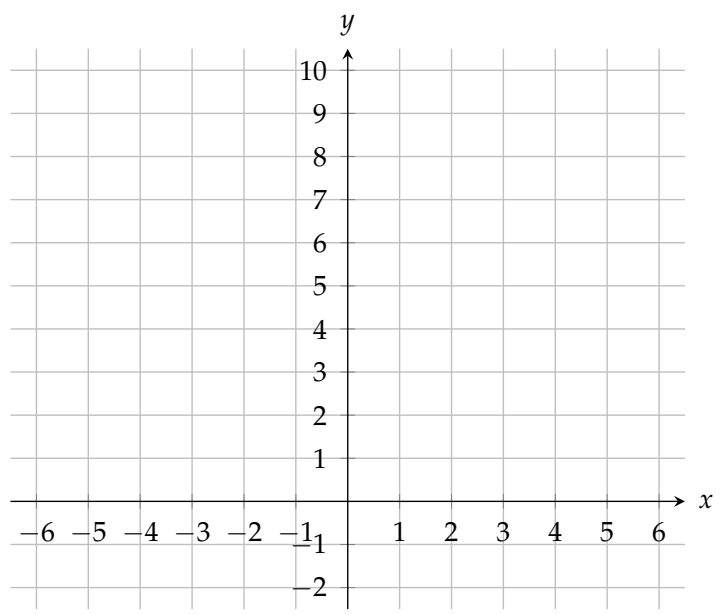

\section{Classifying the graph of a function}

Learning Page To describe the graphs of these functions, there are two important properties to consider:

1. The graph of any function of the form is a

2. The graph of any function of the form , with $a \neq 0$ is a

If $a$ is , the parabola opens and if

the parabola opens 


\section{Interpreting the graphs of two functions}

ใ? Open the Instructor Added Resource which will direct you to a video to complete the following.

The water company has a different monthly pricing plan for residential customers than for business customers. For each pricing plan, cost (in dollars) depends on water used (in hundreds of cubic feet, HCF). Draw in the Residential Plan graph using a solid line and the Business Plan graph using a dashed line. Answer all questions using complete sentences using the context of the problem.

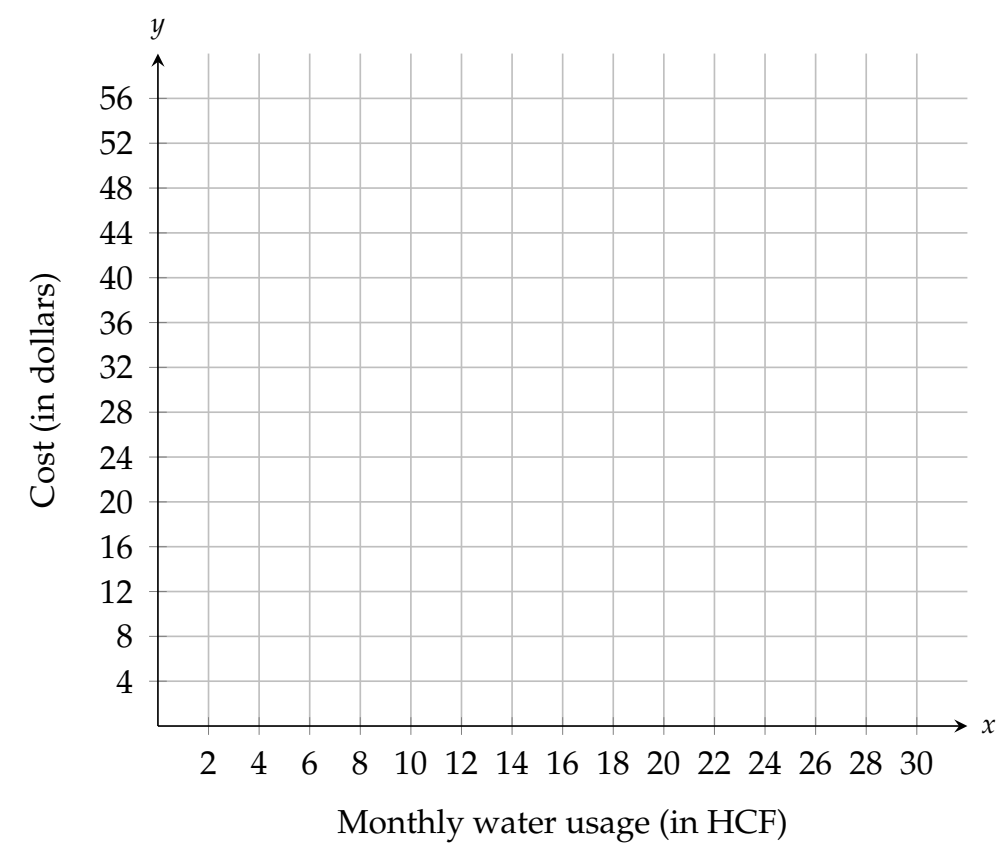

1. If the monthly water usage is $22 \mathrm{HCF}$ which plan costs less?

How much less does it cost than the other plan?

2. For what amount of monthly water usage do the plans cost the same?

If the monthly water usage is less than this amount, which plan costs less?

Notes from Focus Group: 
Notes from Focus Group: 


\section{Module 6}

\section{Contents}

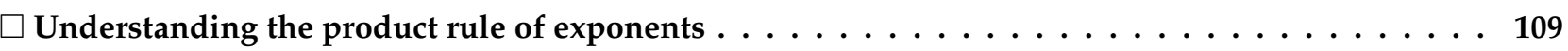

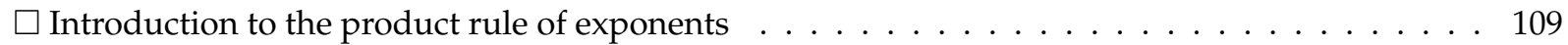

$\square$ Product rule with positive exponents: Univariate $\ldots \ldots \ldots \ldots \ldots$

$\square$ Product rule with positive exponents: Multivariate $\ldots \ldots \ldots \ldots$

$\square$ Understanding the power rules of exponents $\ldots \ldots \ldots \ldots \ldots \ldots$

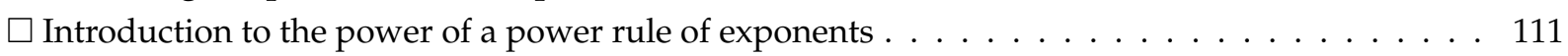

$\square$ Introduction to the power of a product rule of exponents . . . . . . . . . . . . . . 112

$\square$ Introduction to the quotient rule of exponents $\ldots \ldots \ldots \ldots \ldots \ldots \ldots \ldots$

$\square$ Quotient of expressions involving exponents . . . . . . . . . . . . . . . . 114

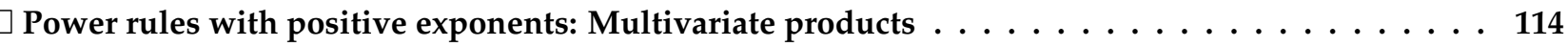

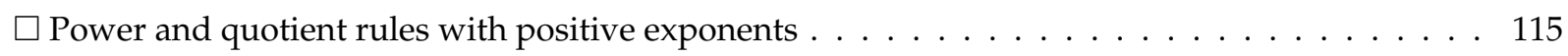

Evaluating expressions with exponents of zero $\ldots \ldots \ldots \ldots \ldots \ldots \ldots \ldots \ldots$

$\square$ Evaluating an expression with a negative exponent: Whole number base $\ldots \ldots \ldots 117$

$\square$ Evaluating an expression with a negative exponent: Negative integer base . . . . . . . 117

$\square$ Evaluating an expression with a negative exponent: Positive fraction base . . . . . . . . . 118

$\square$ Rewriting an algebraic expression without a negative exponent . . . . . . . . . . . . . . . . . . . . . . . . .

$\square$ Introduction to the product rule with negative exponents . . . . . . . . . . . . 120

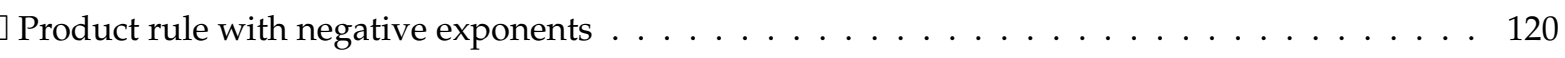

$\square$ Quotient rule with negative exponents: Problem type $1 \ldots \ldots \ldots \ldots \ldots \ldots$

$\square$ Power of a power rule with negative exponents $\ldots \ldots \ldots \ldots \ldots \ldots$

\section{Weekly Checklist}

Complete MALL time.

Work in ALEKS and Notebook at least 3 days a week.

Complete the weekly Module and Notebook pages by the due date.

Attend Focus Group.

Actively participate in Focus Group.

Earn extra credit: Complete 10 topics by 


\section{Understanding the product rule of exponents}

Learning Page The exponents tell us variables to

\section{Introduction to the product rule of exponents}

Learning Page Simplify $x^{6} \cdot x^{5}$

\section{Method 1:}

Using the definition of exponent, we can rewrite this product until we have a single power of $x$.

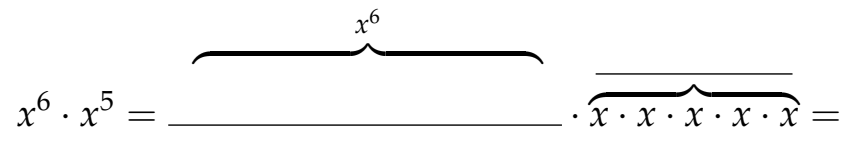

\section{Method 2:}

The method above suggests a rule called the product rule of exponents. It says that for any integers and any we have the following.

$$
a^{m} \cdot a^{n}=
$$

So, when powers with the we

Using the rule with the current problem, we get the following.

$$
x^{6} \cdot x^{5}=x^{6+5}=x^{11}
$$

EXAMPLE: Simplify $m^{2} \cdot m \cdot m^{4}$

Method 1:

$m^{2} \cdot m \cdot m^{4}=\overbrace{m \cdot m}^{m^{2}} \cdot \overbrace{m}^{m_{1}} \cdot \overbrace{m \cdot m \cdot m \cdot m}^{m^{4}}=m^{7}$

Method 2: $m^{2} \cdot m \cdot m^{4}=m^{2+1+4}$

\section{YOU TRY IT:}

83. Simplify $p^{5} \cdot p^{3} \cdot p$. 


\section{Product rule with positive exponents: Univariate}

Watch Video 1: Multiplying Monomials to complete the following.

Multiply the monomials.

1.

2.

3.

\section{EXAMPLE:}

Simplify $-7 w^{3} \cdot 2 w^{6}$.

\section{YOU TRY IT:}

84. Simplify $4 x^{3} \cdot\left(-2 x^{5}\right)$.

Since multiplication is commutative we can reorder the factors.

$$
\begin{aligned}
-7 w^{3} \cdot 2 w^{6} & =-7 \cdot 2 \cdot w^{3} \cdot w^{6} \\
& =-14 \cdot w^{3+6} \\
& =-14 x^{9}
\end{aligned}
$$

\section{Product rule with positive exponents: Multivariate}

$D \quad$ If you have not already done so, watch Video 1: Multiplying Monomials and take notes under the previous topic Product rule with positive exponents: Univariate.

\section{EXAMPLE:}

Simplify $4 y^{2} \cdot 4 x^{5} \cdot\left(-1 x^{6} y^{6}\right)$

Since multiplication is commutative we can reorder the factors.

$$
\begin{aligned}
4 y^{2} \cdot 4 & x^{5} \cdot\left(-1 x^{6} y^{6}\right) \\
& =4 \cdot 4 \cdot(-1) \cdot x^{5} \cdot x^{6} \cdot y^{2} \cdot y^{6} \\
& =-16 \cdot x^{5+6} \cdot y^{2+6} \\
& =-16 x^{11} y^{8}
\end{aligned}
$$

YOU TRY IT:

85. Simplify $3 y^{7} \cdot 4 x^{3} y^{4} \cdot x^{5}$. 


\section{Understanding the power rules of exponents}

Learning Page Carefully read through the example on the Learning Page.

\section{Introduction to the power of a power rule of exponents}

ใ? Open the Instructor Added Resource which will direct you to a video to complete the following.

Simplify

Method 1:

Power of a power rule of exponents

For any integer $n$ and any numbers $a$ and $b$, we have the following.

$$
\left(a^{m}\right)^{n}=
$$

\section{Method 2:}

YOU TRY IT:

86. Simplify $\left(x^{5}\right)^{6}$. 


\section{Introduction to the power of a product rule of exponents}

ใ? Open the Instructor Added Resource which will direct you to a video to complete the following.

Simplify

Method 1:

\section{Power of a product rule of exponents}

For any integer $n$ and any numbers $a$ and $b$, we have the following.

$$
(a b)^{n}=
$$

\section{Method 2:}

\section{YOU TRY IT:}

87. Use the power of a product rule to simplify $(3 p)^{4}$. 


\section{Introduction to the quotient rule of exponents}

ใ? Open the Instructor Added Resource which will direct you to a video to complete the following.

\section{Simplify}

\section{Quotient Rule of Exponents}

$$
\frac{a^{m}}{a^{n}}=
$$

where $m$ and $n$ are and $a$ is any

When powers with the , we the exponents.

Or using the Quotient Rule of Exponents:

YOU TRY IT: Simplify.
88. $\frac{y^{9}}{y^{3}}$
89. $\frac{y^{3}}{y^{9}}$ 


\section{Quotient of expressions involving exponents}

Learning Page Carefully read the example on the Learning Page.

EXAMPLE:

Simplify $\frac{x^{8} y^{6}}{x^{3} y^{4}}$

\section{YOU TRY IT:}

90. Simplify $\frac{a^{5} b^{6}}{a^{7} b^{4}}$.

$$
\begin{aligned}
\frac{x^{8} y^{6}}{x^{3} y^{4}} & =\frac{x^{8}}{x^{3}} \cdot \frac{y^{6}}{y^{4}} \\
& =x^{8-3} y^{6-4} \\
& =x^{5} y^{2}
\end{aligned}
$$

\section{Power rules with positive exponents: Multivariate products}

$\square$ Watch Video 2: Summary of Properties of Exponents to complete the following. NOTE: This may not be the first video that pops up. Select this video from the list of videos on the left of the video box.

\begin{tabular}{|l|l|l|}
\hline \multicolumn{1}{|c|}{ Name } & \multicolumn{1}{|c|}{ Property/Definition } & Example \\
\hline Product & $b^{m} b^{n}=b^{m+n}$ & \\
\hline Quotient & $\frac{b^{m}}{b^{n}}=b^{m-n}$ & \\
\hline $\begin{array}{l}\text { Power of a } \\
\text { power }\end{array}$ & $\left(b^{m}\right)^{n}=b^{m n}$ & \\
\hline $\begin{array}{l}\text { Power of a } \\
\text { product }\end{array}$ & $(a b)^{m}=a^{m} b^{m}$ & \\
\hline $\begin{array}{l}\text { Power of a } \\
\text { quotient }\end{array}$ & $\left(\frac{a}{b}\right)^{m}=\frac{a^{m}}{b^{m}}$ & \\
\hline $\begin{array}{l}\text { Zero } \\
\text { exponent }\end{array}$ & Definition: $b^{0}=1$ for $b \neq 0$ & \\
\hline $\begin{array}{l}\text { Negative } \\
\text { exponent }\end{array}$ & Definition: $b^{-n}=\left(\frac{1}{b}\right)^{n}=\frac{1}{b^{n}}$ for $b \neq 0$ & \\
\hline
\end{tabular}


EXAMPLE: Simplify $\left(-5 x^{2} y\right)^{3}$.

Using the power of a product rule we get

$$
\begin{aligned}
\left(-5 x^{2} y\right)^{3} & =(-5)^{3}\left(x^{2}\right)^{3}\left(y^{1}\right)^{3} \\
& =-125 x^{6} y^{3}
\end{aligned}
$$

YOU TRY IT:

91. Simplify $\left(-3 x y^{4}\right)^{2}$.

\section{Power and quotient rules with positive exponents}

ใ? Open the Instructor Added Resource which will direct you to a video to complete the following.

Simplify. Write your answer using only positive exponents.

1.

2.

YOU TRY IT: Simplify. Write your answer using only positive exponents.
92. $\left(\frac{-16 x y^{5}}{8 x^{3} y}\right)^{3}$
93. $\frac{\left(5 x y^{2}\right)^{3}}{\left(-10 x y^{3}\right)^{2}}$ 


\section{Evaluating expressions with exponents of zero}

$\square$ Watch the video Definition of b to the Zero Power to complete the following.

\section{DEFINITION Definition of $b^{0}$}

Let $b$ be a nonzero real number. Then $b^{0}=1$

Simplify.

1.

4.

Show the example used to explain why $b^{0}=1$ for any nonzero number $b$.
3.

5.
2.

YOU TRY IT: Simplify.

94. $-3^{0}$

95. $(-5)^{0}$ 
Evaluating an expression with a negative exponent: Whole number base

Learning Page For any number $a$ and any whole number we have the following.

Rule 1: $a^{-n}=$

Move to the and change to

Rule 2: $\frac{1}{a^{-n}}=$

Move to the and change to

Evaluating an expression with a negative exponent: Negative integer base

Watch Video 4: Definition of b to a Negative Exponent to complete the following.

DEFINITION Definition of $b^{-n}$

Let $b$ be a an integer. Then and $n$ be

$b^{-n}=$ $=$ $\underline{ }$
Take the of the and change the to

Simplify. Write the answers with positive exponents.

1.
2.

3. 
EXAMPLE: Write all answers with positive exponents. Simplify $8 x^{-2}$.

$$
\begin{aligned}
8 x^{-2} & =\frac{8}{1} \cdot \frac{x^{-2}}{1} \\
& =\frac{8}{1} \cdot \frac{1}{x^{2}} \\
& =\frac{8}{x^{2}}
\end{aligned}
$$

YOU TRY IT:

Simplify. Write all answers with positive exponents.

96. $-7(-2)^{-3}=$

\section{Evaluating an expression with a negative exponent: Positive fraction base}

$\triangleright \quad$ Watch the video Exercise: Simplifying Expressions with Negative Exponents to complete the following.

Simplify and write the answer with positive exponents only.

Write the answer with positive exponents.

EXAMPLE:

Simplify $\left(\frac{-2}{3}\right)^{-4}$.

$$
\begin{aligned}
\left(\frac{-2}{3}\right)^{-4} & =\left(\frac{3}{-2}\right)^{4} \\
& =\left(\frac{3}{-2}\right)\left(\frac{3}{-2}\right)\left(\frac{3}{-2}\right)\left(\frac{3}{-2}\right) \\
& =\frac{81}{16}
\end{aligned}
$$

YOU TRY IT:

97. $\left(\frac{5}{-2}\right)^{-3}=$ 


\section{Rewriting an algebraic expression without a negative exponent}

ใ? Watch the video Rewriting an algebraic expression without a negative exponent to complete the following.

Write the following expressions using only positive exponents and simplify your answers.

1. $\frac{1}{-5 v^{-2}}$

2. $\frac{7}{3 n^{-4}}$

3. $2 x^{-3}$

EXAMPLE:

Write all answers with positive exponents. Simplify $\frac{1}{8 x^{-2}}$.

$$
\begin{aligned}
\frac{1}{8 x^{-2}} & =\frac{1}{8} \cdot \frac{1}{x^{-2}} \\
& =\frac{1}{8} \cdot \frac{x^{2}}{1} \\
& =\frac{x^{2}}{8}
\end{aligned}
$$

\section{YOU TRY IT:}

Simplify. Write all answers with positive exponents.

98. $\frac{2}{-x^{-8}}=$ 


\section{Introduction to the product rule with negative exponents}

Learning Page We'll be using the following rules for exponents.

\section{Product rule:}

For any number $a$ and any integers $m$ and $n$, we have the following.

$$
a^{m} \cdot a^{n}=
$$

So, when powers with the , we the

\section{Negative exponent rule:}

For any number $a$ and any integer $m$, we have the following.

$$
a^{-m}=
$$

\section{EXAMPLE:}

Use only positive exponents in your answer.

Simplify $u^{-4} \cdot u \cdot u^{-6}$.

$$
\begin{aligned}
u^{-4} \cdot u \cdot u^{-6} & =u^{-4+1+(-6)} \quad \text { product rule } \\
& =u^{-9} \\
& =\frac{1}{u} \text { definition of negative exponent }
\end{aligned}
$$

YOU TRY IT:

Simplify. Use only positive exponents in your answer.

99. $x^{-3} \cdot x^{-5} \cdot x^{2}$

\section{Product rule with negative exponents}

Q

Open the Instructor Added Resource which will direct you to a video to complete the following.

Simplify. Use only positive exponents in your answer. 
YOU TRY IT: Simplify. Use only positive exponents in your answer.

100. $5 c^{2} d^{-4} \cdot 2 c^{3} \cdot 6 c^{-2} d^{4}$

\section{Quotient rule with negative exponents: Problem type 1}

Learning Page We will use the following rules for exponents.

\section{Quotient rule:}

For any number $a$ and any $m$ and $n$, we have the following.

$$
\frac{a^{m}}{a^{n}}=
$$

So, when powers with the base, we the

\section{Negative exponent rule:}

For any number $a$ and any integer $m$, we have the following.

$$
a^{-m}=
$$

\section{EXAMPLE:}

Use only positive exponents in your answer.

Simplify $\frac{u^{-4}}{u^{-6}}$.

$$
\begin{aligned}
\frac{u^{-4}}{u^{-6}} & =u^{-4-(-6)} \text { quotient rule } \\
& =u^{2}
\end{aligned}
$$

\section{YOU TRY IT:}

Simplify. Use only positive exponents in your answer.

101. $\frac{x^{-8}}{x^{-5}}$ 


\section{Power of a power rule with negative exponents}

Learning Page We'll be using the following rules for exponents.

\section{Power of a power rule:}

For any number $a$ and any $m$ and $n$, we have the following.

$$
\left(a^{m}\right)^{n}=
$$

Negative exponent rule:

For any nonzero $a$ and any integer $m$, we have the following.

$$
a^{-m}=
$$

\section{EXAMPLE:}

Use only positive exponents in your answer. Simplify $\left(a^{4}\right)^{-3}$.

$$
\begin{aligned}
\left(a^{4}\right)^{-3} & =a^{4 \cdot(-3)} \text { Power of a power rule } \\
& =a^{-12} \\
& =\frac{1}{a^{12}}
\end{aligned}
$$

YOU TRY IT:

Simplify.

102. $\left(x^{-3}\right)^{5}$

103. $\left(y^{-6}\right)^{-7}$

Notes from Focus Group: 
Notes from Focus Group: 


\section{Module 7}

\section{Contents}

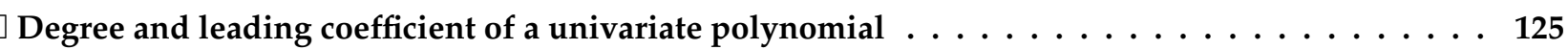

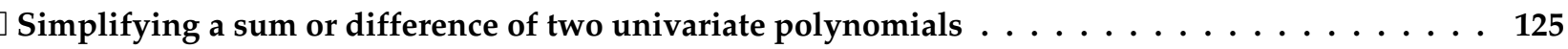

Multiplying a univariate polynomial by a monomial with a negative coefficient $\ldots \ldots \ldots$

$\square$ Multiplying a multivariate polynomial by a monomial $\ldots \ldots \ldots \ldots \ldots$

$\square$ Multiplying binomials with leading coefficients of $1 \ldots \ldots \ldots \ldots \ldots \ldots$

$\square$ Multiplying binomials with leading coefficients greater than $1 \ldots \ldots \ldots \ldots$

$\square$ Multiplying binomials with negative coefficients $\ldots \ldots \ldots \ldots \ldots \ldots$

$\square$ Multiplication involving binomials and trinomials in one variable . . . . . . . . . . . . . . . . . . . . . . . . . . . . .

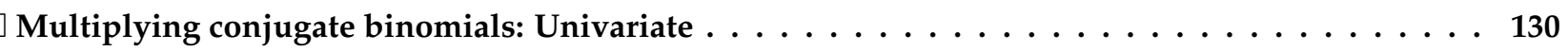

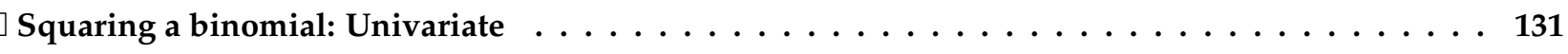

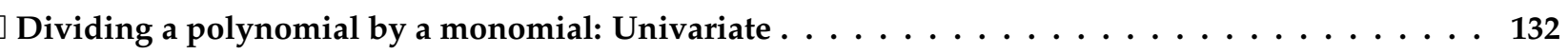

$\square$ Polynomial long division: Type $1 \ldots \ldots \ldots \ldots \ldots \ldots \ldots \ldots \ldots$

$\square$ Polynomial long division: Type $2 \ldots \ldots \ldots \ldots \ldots \ldots \ldots \ldots$

$\square$ Greatest common factor of 2 numbers $\ldots \ldots \ldots \ldots \ldots \ldots \ldots \ldots \ldots$

$\square$ Greatest common factor of three univariate monomials . . . . . . . . . . . . . . . 136

$\square$ Greatest common factor of two multivariate monomials $\ldots \ldots \ldots 137$

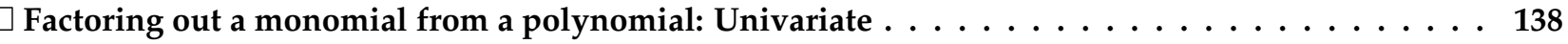

$\square$ Factoring out a binomial from a polynomial: GCF factoring, basic . . . . . . . . . . . . . . 138

$\square$ Factoring a univariate polynomial by grouping: Problem type $1 \ldots \ldots \ldots \ldots$

$\square$ Factoring a univariate polynomial by grouping: Problem type $2 \ldots \ldots$. . . . . . . . 140

\section{Weekly Checklist}

Complete MALL time.

Work in ALEKS and Notebook at least 3 days a week.

Complete the weekly Module and Notebook pages by the due date.

Attend Focus Group.

Actively participate in Focus Group.

Earn extra credit: Complete 10 topics by 


\section{Degree and leading coefficient of a univariate polynomial}

Watch Video 2: Degree, Descending Order, Leading Term, and Coefficients to complete the following.

Given the polynomial

a. List the terms of the polynomial.

b. Write the polynomial in descending order.

c. State the degree of the polynomial and the leading coefficient.

Degree:___ Leading coefficient:

Given the polynomial

a. Identify the degree of each term.

b. Identify the degree of the polynomial.

\section{Simplifying a sum or difference of two univariate polynomials}

$\square$ Watch Video 6: Subtracting Polynomials Horizontally and Vertically to complete the following. NOTE: This may not be the first video that pops up. Select this video from the list of videos on the left of the video box.

DEFINITION Subtraction of Polynomials

If $A$ and $B$ are then

Subtract the polynomials 
YOU TRY IT: Add the polynomial.

104. $\left(-3 x^{5}+2 x^{3}+5\right)+\left(7 x^{5}-8 x^{3}+9\right)$

\section{Multiplying a univariate polynomial by a monomial with a negative coefficient}

$\square$ Watch Video 2: Multiplying a Monomial by a Polynomial to complete the following. NOTE: This may not be the first video that pops up. Select this video from the list of videos on the left of the video box.

Multiply the polynomials.

1.

2.

\section{EXAMPLE:}

Rewrite without parentheses.

$$
\begin{aligned}
-6 y^{6} & \left(-2 y^{2}-5 y+3\right) \\
& =\left(-6 y^{6}\right)\left(-2 y^{2}\right)+\left(-6 y^{6}\right)(-5 y)+\left(-6 y^{6}\right)(3) \\
& =12 y^{8}+30 y^{7}-18 y^{6}
\end{aligned}
$$

\section{YOU TRY IT:}

Multiply the polynomials.

105. $-5 x^{3}\left(2 x^{2}-7 x+6\right)$ 


\section{Multiplying a multivariate polynomial by a monomial}

If you have not already done so, watch Video 2: Multiplying a Monomial by a Polynomial and take notes under the previous topic Multiplying a univariate polynomial by a monomial with a negative coefficient.

\section{EXAMPLE:}

Rewrite without parentheses.

$$
\begin{aligned}
& \left(14 x y^{2}-5 x^{2} y+x^{3} y\right)(-2 y) \\
& \begin{aligned}
\left(14 x y^{2}-5 x^{2} y+x^{3} y\right)(-2 y) \\
\quad=\left(14 x y^{2}\right)(-2 y)-\left(5 x^{2} y\right)(-2 y)+\left(x^{3} y\right)(-2 y) \\
\quad=-28 x y^{3}+10 x^{2} y^{2}-2 x^{3} y^{2}
\end{aligned}
\end{aligned}
$$

\section{YOU TRY IT:}

Rewrite without parentheses.

106. $-4 x^{3} y^{7} z\left(2 x y^{2} z^{4}-\frac{1}{2} x^{5} y\right)$

\section{Multiplying binomials with leading coefficients of 1}

$\square$ Watch Video 3: Multiplying Binomials to complete the following.

Multiply the polynomials.

YOU TRY IT: Multiply the polynomials.

107. $(x-3)(x+5)$ 


\section{Multiplying binomials with leading coefficients greater than 1}

$\triangleright$ Watch Exercise: Multiplying Binomials to complete the following.

Multiply the polynomials by using the distributive property.

\section{EXAMPLE:}

Multiply the polynomials. $(4 x-7)(2 x+1)$

$$
(4 x-7)(2 x+1)
$$$$
=4 x(2 x)+4 x(1)+(-7)(2 x)+(-7)(1) \text { Distributive Property }
$$$$
=8 x^{2}+4 x-14 x-7
$$$$
=8 x^{2}-10 x-7
$$

\section{YOU TRY IT:}

Multiply the polynomials.

108. $(2 x-3)(3 x+5)$

\section{Multiplying binomials with negative coefficients}

Learning Page Carefully read through the example on the Learning Page.

EXAMPLE:

Multiply the polynomials $(-2 x+1)(-x+7)$.

$$
\begin{aligned}
(-2 x & +1)(-x+7) \\
& =(-2 x)(-x)+(-2 x)(7)+1(-x)+(1)(7) \\
& =2 x^{2}-14 x-x+7 \\
& =2 x^{2}-15 x+7
\end{aligned}
$$

YOU TRY IT: Multiply the polynomials.

109. $(2 x-3)(-3 x+5)$ 
Multiplication involving binomials and trinomials in one variable

Watch Video 5: Multiplying a Binomial by a Trinomial to complete the following.

Multiply the polynomials.

EXAMPLE: Multiply the polynomials $\left(2 y^{2}-5 y-1\right)\left(3 y^{2}-4 y+4\right)$.

$$
\begin{aligned}
& \left(2 y^{2}-5 y-1\right)\left(3 y^{2}-4 y+4\right) \\
& =2 y^{2}\left(3 y^{2}-4 y+4\right)-5 y\left(3 y^{2}-4 y+4\right)-1\left(3 y^{2}-4 y+4\right) \quad \text { Distributive Property } \\
& =2 y^{2}\left(3 y^{2}\right)+2 y^{2}(-4 y)+2 y^{2}(4)-5 y\left(3 y^{2}\right)-5 y(-4 y)-5 y(4)-1\left(3 y^{2}\right)-1(-4 y)-1(4) \quad \begin{array}{c}
\text { Distributive } \\
\text { Property }
\end{array} \\
& =6 y^{4}-8 y^{3}+8 y^{2}-15 y^{3}+20 y^{2}-20 y-3 y^{2}+4 y-4 \\
& =6 y^{4}-23 y^{3}+25 y^{2}-16 y-4 \quad \text { Combine like terms }
\end{aligned}
$$

YOU TRY IT: Multiply the polynomials.

110. $(x-3)\left(3 x^{2}+4 x-5\right)$ 


\section{Multiplying conjugate binomials: Univariate}

$\square$ Watch Video 7: Multiplying Conjugates to complete the following. NOTE: This may not be the first video that pops up. Select this video from the list of videos on the left of the video box.

FORMULA Special Case Products

$(a+b)(a-b)=\ldots \quad$ The product of conjugates results in a

Multiply the polynomials. Show the work.

1.

2.

EXAMPLE:

Multiply the polynomials $(5-4 x)(5+4 x)$.

$$
\begin{aligned}
(5-4 x)(5+4 x) & =5^{2}-(4 x)^{2} \\
& =25-16 x^{2}
\end{aligned}
$$

YOU TRY IT: Multiply the polynomials.

111. $(3 x+4)(3 x-4)$ 


\section{Squaring a binomial: Univariate}

Watch Video 8: Squaring Binomials to complete the following. NOTE: This may not be the first video that pops up. Select this video from the list of videos on the left of the video box.

FORMULA Special Case Products

- $(a+b)^{2}=$

The square of a binomial results in a

- $(a-b)^{2}=$

Multiply.

1.

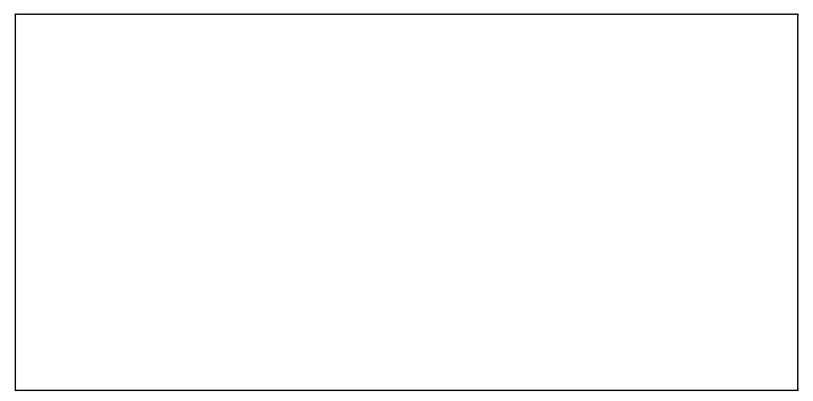

2.

EXAMPLE: Multiply $(4-3 x)^{2}$.

$$
\begin{aligned}
(4-3 x)^{2} & =(4-3 x)(4-3 x) \\
& =16-12 x-12 x+9 x^{2} \\
& =16-24 x+9 x^{2}
\end{aligned}
$$

YOU TRY IT: Multiply.

112. $(3 x-2)^{2}$ 
Module 7

\section{Dividing a polynomial by a monomial: Univariate}

$\square$ Watch Video 1: Dividing a Polynomial by a Monomial to complete the following.

\section{Divide.}

1.

2.

YOU TRY IT: Divide.

113. $\frac{3 x^{4}-6 x^{3}+9 x}{3 x^{2}}$ 


\section{Polynomial long division: Type 1}

$\square$ Watch the video Dividing Polynomials Using Long Division to complete the following.

Divide the polynomials by using long division. Check your answer by multiplication.

Check: $($ divisor $)($ quotient $)+$ remainder $=$ dividend

The quotient is

The remainder is

EXAMPLE:

Use polynomial long division to evaluate:

$$
\begin{aligned}
& x-2) \frac{4 x^{2}+15 x+30}{4 x^{3}+7 x^{2}-3} \\
& -4 x^{3}+8 x^{2} \\
& \frac{-15 x^{2}+30 x}{30 x-3} \\
& \frac{-30 x+60}{57}
\end{aligned}
$$

The quotient is $4 x^{2}+15 x+30$.

The remainder is 57 .

\section{YOU TRY IT:}

Use polynomial long division to evaluate:

114. $\left(2 x^{3}+3 x^{2}+4 x+3\right) \div(x+3)$ 


\section{Polynomial long division: Type 2}

$\square$ Watch the video Video 3: Long Division of Polynomials to complete the following. NOTE: This may not be the first video that pops up. Select this video from the list of videos on the left of the video box.

Check: (divisor) $($ quotient $)+$ remainder $=$ dividend

The quotient is

The remainder is

EXAMPLE:

Use polynomial long division to evaluate:

$\left(x^{4}+3 x^{3}+x-5\right) \div\left(x^{2}-3\right)$

$$
\begin{array}{r}
x^{2}+3 x+3 \\
\cline { 2 - 2 } \begin{array}{r}
x^{4}+3 x^{3}+3 x^{2} \\
-x^{4}+3 x^{2}
\end{array} \\
\frac{-3 x^{3}+3 x^{2}+x}{3 x^{2}+10 x-5} \\
\frac{-3 x^{2}+9}{10 x+4}
\end{array}
$$

The quotient is $x^{2}+3 x+3$.

The remainder is $10 x+4$.

\section{YOU TRY IT:}

Use polynomial long division to evaluate:

115. $\left(6 x^{3}+5 x^{2}+7 x-1\right) \div(3 x+1)$ 


\section{Greatest common factor of 2 numbers}

2?

Open the Instructor Added Resource which will direct you to a video to complete the following.

\section{Greatest Common Factor (GCF)}

The GCF is the

Find the GCF of the following numbers.

- $\mathrm{GCF}($

Factors of

Factors of

Common factors:

$\mathrm{GCF}(\longrightarrow)=$
- $\mathrm{GCF}($

Factors of

Factors of

Common factors:

GCF(

$=$
- $\mathrm{GCF}($

Factors of

Factors of

Common factors:

$\operatorname{GCF}(-)=$

YOU TRY IT: Find the GCF of the two numbers

116. $\operatorname{GCF}(35,28)$ 


\section{Greatest common factor of three univariate monomials}

$\square$ Watch Video 1: Identifying the Greatest Common Factor to complete the following.

Identify the greatest common factor.

The greatest common factor of a polynomial is the greatest common factor that

1. For the polynomial the GCF $=$

List the factors of each term:

Circle the factors in each term that must be in the GCF.

II Pause the video and try this yourself.

2.

List the factors of each term: Circle the factors in each term that must be in the GCF of these terms.

The GCF of this polynomial is

$\square$ Play the video and check your answer. 
EXAMPLE: Find the GCF of the terms of the polynomial $36 x^{7}+90 x^{6}-72 x^{4}$

First, find the GCF of the coefficients, 36, 90, and 72 by listing the factors of each.

$$
\begin{aligned}
& 36=2 \cdot 2 \cdot 3 \cdot 3=2^{2} \cdot 3^{2} \\
& 90=2 \cdot 3 \cdot 3 \cdot 5=2 \cdot 3^{2} \cdot 5 \\
& 72=2 \cdot 2 \cdot 2 \cdot 3 \cdot 3=2^{3} \cdot 3^{2}
\end{aligned}
$$

The GCF is $2 \cdot 3 \cdot 3=2 \cdot 3^{2}=18$

Faster method:

- the $\operatorname{GCF}(36,90,72)$ is made up of the smallest power of each of the common factors.

- 36, 90, and 72 have factors of 2 and 3 in common.

- The smallest power of 2 in any list is 1 and the smallest power of 3 in any list is 2 .

Thus, the GCF is $2^{1} \cdot 3^{2}=18$.

Next, find the GCF of the variables, $x^{7}, x^{6}$, and $x^{4}$.

The common factor for each of these is $x$ and the smallest power of $x$ is $x^{4}$. So, the $\operatorname{GCF}\left(x^{7}, x^{6}, x^{4}\right)=x^{4}$.

The GCF of the terms of the polynomial is $18 x^{4}$.
YOU TRY IT:

Find the GCF.

117. $20 x^{5}, 60 x^{3}$, and $4 x^{2}$ 
YOU TRY IT: Find the greatest common factor of the two terms.

118. $14 a^{3} b^{5}$ and $49 a b^{7}$

\section{Factoring out a monomial from a polynomial: Univariate}

$\square$ Watch Video 2: Factor Out the Greatest Common Factor to complete the following.

Factor out the greatest common factor (GCF).

YOU TRY IT: Factor out the GCF.

119. $14 x^{4}-7 x^{3}+21 x$

\section{Factoring out a binomial from a polynomial: GCF factoring, basic}

$\square$ Watch the video Factoring Out a Binomial Factor to complete the following.

Factor out the greatest common factor.

What is the common factor in all three terms? 
EXAMPLE:

Factor out the GCF.

$4 x^{3}\left(x^{2}+3\right)-2 x\left(x^{2}+3\right)+\left(x^{2}+3\right)$

$\left(x^{2}+3\right)$ is the factor common to all 3 terms so we write:

$$
\begin{gathered}
4 x^{3}\left(x^{2}+3\right)-2 x\left(x^{2}+3\right)+\left(x^{2}+3\right) \\
=\left(x^{2}+3\right)\left(4 x^{3}-2 x+1\right)
\end{gathered}
$$

YOU TRY IT:

Factor out the GCF.

120. $y^{3}(y+2)-y(y+2)-9(y+2)$

\section{Factoring a univariate polynomial by grouping: Problem type 1}

Watch Video 7: Factoring by Grouping to complete the following. NOTE: This may not be the first video that pops up. Select this video from the list of videos on the left of the video box.

Factor by grouping.

What is the first pair of terms?

What is the GCF of these terms?

What is the second pair of terms?

What is the GCF of these terms?

Check: 
EXAMPLE: Factor by grouping.

$$
3 x^{3}-2 x^{2}+12 x-8
$$

In the first pair of terms the GCF is $x^{2}$.

In the second pair of terms the GCF is 4 .

$$
3 x^{3}-2 x^{2}+12 x-8=x^{2}(3 x-2)+4(3 x-2)
$$

$3 x-2$ is the common factor in these two terms.

$$
\begin{aligned}
3 x^{3}-2 x^{2}+12 x-8 & =x^{2}(3 x-2)+4(3 x-2) \\
& =(3 x-2)\left(x^{2}+4\right)
\end{aligned}
$$

YOU TRY IT: Factor by grouping.

121. $y^{3}+3 y^{2}-3 y-9$

\section{Factoring a univariate polynomial by grouping: Problem type 2}

If you have not completed the Notebook entry for the previous topic, watch Video 7: Factoring by Grouping and complete the notes now.

EXAMPLE: Factor by grouping.

$$
12 x^{4}-20 x^{3}-3 x+5
$$

In the first pair of terms the GCF is $4 x^{3}$.

$$
12 x^{4}-20 x^{3}-3 x+5=4 x^{3}(3 x-5)-3 x+5
$$

$3 x-5$ is not quite the same as the last two terms, $-3 x+5$.

\section{Factor out -1 .}

If we factor -1 out of $-3 x+5$ we get

$$
-3 x+5=-1(3 x-5) \text {. }
$$

Then,

$$
\begin{aligned}
12 x^{4}-20 x^{3}-3 x+5 & =4 x^{3}(3 x-5)-1(3 x-5) \\
& =(3 x-5)\left(4 x^{3}-1\right)
\end{aligned}
$$

YOU TRY IT: Factor by grouping.

122. $2 y^{3}-8 y^{2}-y+4$

Notes from Focus Group: 
Notes from Focus Group: 


\section{Module 8-Review}

To help you review for your upcoming exam, this module contains all of the topics from the modules since the last exam. Topics that you have already mastered will not appear in your carousel.

Complete this module before you take the ALEKS exam.

Each exam has two parts.

- The ALEKS exam (100 pts)

- The ALEKS exam must be taken in the MALL.

- The ALEKS exam is a Comprehensive Knowledge Check.

- Your score is the number of topics you have mastered out of the number of topics you should have mastered by this point.

- If you lose topics on your ALEKS exam, your Review Module completion grade will not change.

- Your scratch work for the ALEKS exam must be numbered and turned in through Blackboard.

- The Written exam (25 pts)

- Take your written exam in class the day of your focus group.

○ To study for the written exam:

- Rework your old Focus Group assignments.

- Rework any topics in ALEKS you may have lost on the ALEKS exam.

\begin{tabular}{|l|l|}
\hline & Score \\
\hline ALEKS Exam & \\
\hline Written Exam & \\
\hline
\end{tabular}




\section{Module 9}

\section{Contents}

$\square$ Factoring a quadratic with leading coefficient $1 \ldots \ldots \ldots \ldots \ldots \ldots \ldots$

$\square$ Factoring a quadratic with a negative leading coefficient $\ldots \ldots \ldots \ldots \ldots$

Factoring out a constant before factoring a quadratic $\ldots \ldots \ldots \ldots \ldots \ldots$

$\square$ Factoring a quadratic with leading coefficient greater than 1: Problem type $1 \ldots \ldots$. . . . . 146

Factoring a quadratic with leading coefficient greater than 1 : Problem type $2 \ldots \ldots 146$

$\square$ Factoring a perfect square trinomial with leading coefficient $1 \ldots \ldots \ldots \ldots \ldots$

$\square$ Factoring a perfect square trinomial with leading coefficient greater than $1 \ldots \ldots$. . . . . . . . . . . . . 149

Factoring a product of a quadratic trinomial and a monomial $\ldots \ldots \ldots \ldots \ldots$

Factoring a difference of squares in one variable: Basic . . . . . . . . . . . . . . 150

$\square$ Factoring a difference of squares in one variable: Advanced . . . . . . . . . . . . . 151

$\square$ Solving an equation written in factored form $\ldots \ldots \ldots \ldots \ldots \ldots \ldots \ldots \ldots$

$\square$ Finding the roots of a quadratic equation with leading coefficient $1 \ldots \ldots \ldots \ldots 2$

$\square$ Finding the roots of a quadratic equation of the form $a x^{2}+b x=0 \ldots \ldots \ldots$

$\square$ Finding the roots of a quadratic equation with leading coefficient greater than $1 \ldots \ldots$

Solving a quadratic equation needing simplification $\ldots \ldots \ldots \ldots \ldots \ldots$

Solving a word problem using a quadratic equation with rational roots $\ldots \ldots \ldots \ldots$

$\square$ Writing a quadratic equation given the roots and the leading coefficient $\ldots \ldots \ldots \ldots$

\section{Weekly Checklist}

Complete MALL time.

Work in ALEKS and Notebook at least 3 days a week.

Complete the weekly Module and Notebook pages by the due date.

Attend Focus Group.

Actively participate in Focus Group.

Earn extra credit: Complete 10 topics by 


\section{Factoring a quadratic with leading coefficient 1}

$\square$ Watch Video 9: Factoring Trinomials with a Leading Coefficient of 1 to complete the following.

Factor completely.

List the factors of the 3rd term in the trinomial.

Why were the factors -3 and -7 chosen?

Check:

YOU TRY IT: Factor completely.

123. $x^{2}-12 x+27$

\section{Factoring a quadratic with a negative leading coefficient}

$\square$ Watch Video 7: Factoring a Trinomial with a Negative Leading Coefficient to complete the following.

Factor the trinomial completely by using any method. Remember to look for a common factor first.

The GCF of the terms is Since the first term is we factor out a

To factor into two binomials we need two numbers whose product is and sum is 
YOU TRY IT: Factor completely.

124. $-x^{2}+2 x+3$

\section{Factoring out a constant before factoring a quadratic}

$D$ Watch the video Exercise: Factoring a Trinomial with a Leading Coefficient of 1 and a GCF to complete the following. NOTE: This may not be the first video that pops up. Select it from the list of videos in the video box.

Factor the trinomial completely by using any method. Remember to look for a common factor first.

The GCF of the terms is Since the first term is we factor out a

To factor into two binomials we need two numbers whose product is and sum is

\section{EXAMPLE:}

Factor completely. $\quad 4 x^{2}+8 x-4$

We factor out 4, the GCF of the trinomial to get

$$
4 x^{2}+8 x-4=4\left(x^{2}+2 x-1\right) .
$$

When we apply the trial and error or ac-method we find that there are no factors of -1 that add to +2 .

The trinomial cannot be factored any further and we say that $x^{2}+2 x-1$ is prime.

$4\left(x^{2}+2 x-1\right)$ is the final answer.

\section{YOU TRY IT:}

Factor completely.

125. $6 a^{2}+21 a-12$ 


\section{Factoring a quadratic with leading coefficient greater than 1: Problem type 1}

$\square$ Watch Video 5: Factoring a Trinomial with Leading Coefficient not Equal to 1 (Trial-and-Error Method) to complete the following.

Factor.

\section{Factoring a quadratic with leading coefficient greater than 1: Problem type 2}

$\square$ Watch Video 4: Factoring a Trinomial by the Trial-and-Error Method (Leading Coefficient Not Equal to 1) to complete the following.

Factor completely.

The GCF of the three terms is

The leading terms of the binomials must be

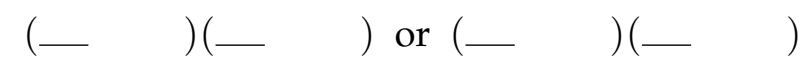

List the factors of the last term of the trinomial. 
EXAMPLE: Factor completely $6 x^{2}-x-15$.

Using the trial-and-error method for factoring, we list the factors of 6 and -15 .

$\begin{array}{rl}6 & -15 \\ 1,6 & -1,15 \\ 2,3 & 1,-15 \\ & -3,5 \\ & 3,-5\end{array}$

1. $(\underline{3 x}-\underline{3})(\underline{2 x}+\underline{5})$

2. $(\underline{3 x}+\underline{3})(\underline{2 x}-\underline{5})$

3. $(\underline{3 x}-\underline{5})(\underline{2 x}+\underline{3})$

4. $(\underline{3 x}+\underline{5})(\underline{2 x}-\underline{3})$

We discard options 1 and 2 because the binomials $(3 x \pm 3)$ have a common factor of 3 . This is NOT possible because the terms of $6 x^{2}-x+15$ do not have a common factor.

- The first term of the binomials must be factors of 6 .

- The last term of the binomials must be factors of -15 .

- The sum of the product of the outer and the product of the inner terms of the binomials must add to $-1 x$.

Next we test \#3. The sum of the product of the outer and the product of the inner terms of the binomials must be $-1 x$.

Outer: $-5(2 x)=-10 x \quad$ Inner: $3 x(3)=9 x$

Sum: $-10 x+9 x=-x$

Since $-1 x$ is close to zero we choose factors that are "close" together. Let's try 2 and 3 as factors of 6 and -3 and 5 as factors of -15 . This gives us the following options:

This pair of binomials meets all of our needs. Thus, the factored form of $6 x^{2}-x+15$ is

$$
(3 x-5)(2 x+3)
$$

127. $2 x^{2}-7 x-15$ 


\section{Factoring a perfect square trinomial with leading coefficient 1}

$\triangleright \quad$ Watch Video 10: Recognizing and Factoring a Perfect Square Trinomial to complete the following. NOTE: This may not be the first video that pops up. Select this video from the list of videos on the left of the video box.

Factor completely.

The GCF is

This trinomial is a perfect square trinomial. Before we factor this, let's remember how to square a binomial. $(a+b)^{2}=$

We can rewrite $4 x^{2}+12 x+9$ as

This can be factored as

Check:

\section{EXAMPLE:}

Factor completely. $\quad x^{2}-14 x+49$

This is a perfect square trinomial if it fits the form:

$$
(a)^{2}+a(a)(b)+(b)^{2}
$$

We can write

$$
x^{2}-14 x+49=(x)^{2}+2(x)(-7)+(-7)^{2}
$$

which fits the form of a perfect square trinomial. Thus,

$$
x^{2}-14 x+49=(x+(-7))^{2}=(x-7)^{2}
$$

YOU TRY IT: Factor completely.

128. $x^{2}-10 x+25$ 


\section{Factoring a perfect square trinomial with leading coefficient greater than 1}

If you have not completed the Notebook entry for the previous topic, watch Video 10: Recognizing and Factoring a Perfect Square Trinomial and complete the notes now.

\section{EXAMPLE:}

Factor completely. $\quad 9 x^{2}-24 x+16$

This is a perfect square trinomial if it fits the form:

$$
(a)^{2} 2 a(a)(b)+(b)^{2}
$$

We can write

$$
9 x^{2}-24 x+16=(3 x)^{2}+2(3 x)(-4)+(-4)^{2}
$$

which fits the form of a perfect square trinomial.

Thus,

$$
9 x^{2}-24 x+16=(3 x+(-4))^{2}=(3 x-4)^{2}
$$

YOU TRY IT: Factor completely.

129. $16 x^{2}-20 x+25$

\section{Factoring a product of a quadratic trinomial and a monomial}

Watch Video 6: Factoring a Trinomial Using the Trial-and-Error Method and when Rearranging Terms is Necessary to complete the following. NOTE: This may not be the first video that pops up. Select it from the list of videos in the video box.

Factor completely.

The GCF is 
EXAMPLE: Factor completely.

$$
7 x^{5}-14 x^{4}-7 x^{3}
$$

The GCF is $7 x^{3}$. Factoring out $7 x^{3}$ we have

$$
7 x^{5}-14 x^{4}-7 x^{3}=7 x^{3}\left(x^{2}-2 x-1\right)
$$

Next we try to factor $x^{2}-2 x-1$.

Using trial-and-error we look for factors of -1 that sum to -2 .

-1 and 1 is the only pair of factors for -1 and

$-1+1 \neq-2$.

Thus, $x^{2}-2 x-1$ cannot be factored any further and we say that $x^{2}-2 x-1$ is prime.

$7 x^{3}\left(x^{2}-2 x-1\right)$ is completely factored.
YOU TRY IT:

130. Factor completely. $-20 x^{3}+34 x^{2}-6 x$

\section{Factoring a difference of squares in one variable: Basic}

$\square$ Watch Video 1: Introduction to Factoring a Difference of Squares and a Sum of Squares to complete the following.

Factor completely if possible.

1.

First, we remember taking the product of conjugates such as

The result is the or $a^{2}-b^{2}$.
2.

A sum of squares

$$
a^{2}+b^{2} \text { is }
$$


EXAMPLE:

Factor completely, if possible. $100-y^{2}$

We can write $100-y^{2}=(10)^{2}-(y)^{2}$.

This shows that it is a difference of two squares which factors as

$$
(10-y)(10+y)
$$

YOU TRY IT: Factor completely, if possible

131. $x^{2}-49$

\section{Factoring a difference of squares in one variable: Advanced}

$D$ Watch Video 2: Factoring a Difference of Squares to complete the following. NOTE: This may not be the first video that pops up. Select this video from the list of videos on the left of the video box.

Factor completely.

\begin{tabular}{|ll|}
\hline Perfect squares & Perfect Squares \\
\hline $1^{2}=$ & $\left(x^{1}\right)^{2}=$ \\
$2^{2}=$ & $\left(x^{2}\right)^{2}=$ \\
$3^{2}=$ & $\left(x^{3}\right)^{2}=$ \\
$4^{2}=$ & $\left(x^{4}\right)^{2}=$ \\
$5^{2}=$ & \\
$6^{2}=$ & \\
$7^{2}=$ & \\
$8^{2}=$ & \\
$9^{2}=$ & \\
$10^{2}=$ & \\
$11^{2}=$ & \\
$12^{2}=$ & \\
$13^{2}=$ & \\
\hline
\end{tabular}




\section{Solving an equation written in factored form}

$\square$ Watch Video 2: Solving a Quadratic Equation Using the Zero Product Rule to complete the following.

\section{PROPERTY Zero Product Rule}

If $a b=0$, then or

Solve the equation

EXAMPLE:

Solve the equation $-2 x(x-4)(8+x)=0$.

$$
-2 x(x-4)(8-x)=0
$$

$$
\begin{aligned}
& -2 x=0 \text { or } x-4=0 \text { or } 8-x=0 \\
& x=0 \quad \text { or } \quad x=4 \quad \text { or } \quad 8=x
\end{aligned}
$$

The solution is $x=0,4,8$.
YOU TRY IT:

132. Solve the equation $(x+5)(2 x-3)=0$.

\section{Finding the roots of a quadratic equation with leading coefficient 1}

$\square$ Watch Video 4: Solving a Quadratic Equation Using the Zero Product Rule to complete the following.

Solve the equation. 
EXAMPLE: Solve equation $x^{2}-11 x=-18$.

First we MUST rewrite the equation so that it is set $=0$.

$$
\begin{aligned}
x^{2}-11 x & =-18 \\
x^{2}-11 x+18 & =0
\end{aligned}
$$

Using the trail-and-error method of factoring, we have

$$
x^{2}-11 x+18=(x-9)(x-2)
$$

We solve the equation as follows:

$$
\begin{gathered}
x^{2}-11 x+18=0 \\
(x-9)(x-2)=0 \\
x-9=0 \text { or } x-2=0 \\
x=9 \text { or } x=2
\end{gathered}
$$

The solution is $x=9,2$.

\section{YOU TRY IT:}

133. Solve the equation $x^{2}+4 x-21=0$.

134. Solve the equation $x^{2}+8 x=-15$.

\section{Finding the roots of a quadratic equation of the form $a x^{2}+b x=0$}

$\square$ Watch Video 5: Solving a Quadratic Equation using the Zero Product Rule to complete the following.

Solve the equation.

EXAMPLE:

Solve the equation $2 x^{2}+8 x=0$.

$$
\begin{aligned}
2 x^{2}+8 x & =0 \\
2 x(x+4) & =0
\end{aligned}
$$

$$
\begin{aligned}
2 x & =0 & \text { or } & & x+4 & =0 \\
x & =0 & \text { or } & & x & =-4
\end{aligned}
$$

The solution is $x=0,-4$.

\section{YOU TRY IT:}

135. Solve the equation $4 x^{2}-20 x=0$. 


\section{Finding the roots of a quadratic equation with leading coefficient greater than 1}

Open the e-book to complete the following.

\section{Solving a Quadratic Equation by Factoring}

Step 1 Write the equation in the form

Step 2 completely.

Step 3 Apply the That is, each factor equal to and solve the resulting equations.

If necessary for step 2, review the topic "Factoring a quadratic with leading coefficient greater than 1: Problem type $2 . "$

\section{EXAMPLE:}

Solve the equation $15 x^{2}+7 x-4=0$.

$$
\begin{aligned}
& 15 x^{2}+7 x-4=0 \\
& (5 x+4)(3 x-1)=0 \\
& \begin{aligned}
5 x+4 & =0 & & \text { or } & 3 x-1 & =0 \\
x & =-\frac{4}{5} & & \text { or } & x & =\frac{1}{3}
\end{aligned}
\end{aligned}
$$

The solution is $x=-\frac{4}{5}, \frac{1}{3}$.

\section{YOU TRY IT:}

136. Solve the equation $4 x^{2}-x-3=0$.

137. Solve the equation $9 x^{2}+3 x-2=0$. 


\section{Solving a quadratic equation needing simplification}

$\square$ Watch Video 6: Solving a Quadratic Equation Using the Zero Product Rule to complete the following.

Solve the equation.

EXAMPLE: Solve: $2 x^{2}-x-3=(x+1)^{2}$.

YOU TRY IT:

$$
\begin{aligned}
& 2 x^{2}-x-3=(x+1)^{2} \\
& 2 x^{2}-x-3=x^{2}+2 x+1 \\
& x^{2}-3 x-4=0
\end{aligned}
$$

138. Solve: $2 x^{2}+x=(x-2)^{2}-10$

using the trial-and-errormethod we get

$$
\begin{array}{r}
(x-4)(x+1)=0 \\
x=4,-1
\end{array}
$$




\section{Solving a word problem using a quadratic equation with rational roots}

$\square$ Watch Video 10: Solving a Geometry Application Using a Quadratic Equation (Area of a Rectangle) to complete the following.

The area of a rectangular field is The length is

Find the

\section{YOU TRY IT:}

139. The length of a rectangular photograph is 7 in more than the width. If the area is $78 \mathrm{in}^{2}$, what are the dimensions of the photograph? 


\section{Writing a quadratic equation given the roots and the leading coefficient}

ใ्? Open the Instructor Added Resource which will direct you to a video to complete the following.

Write the quadratic equation whose roots are and and whose leading coefficient is

Use the letter $x$ to represent the variable.

\section{YOU TRY IT:}

140. Write the quadratic equation whose roots are -7 and 2 and whose leading coefficient is -1 . Use the letter $x$ to represent the variable.

Notes from Focus Group: 
Module 9

Notes from Focus Group: 


\section{Module 10}

\section{Contents}

$\square$ Evaluating a rational function: Problem type $1 \ldots \ldots \ldots \ldots \ldots \ldots \ldots \ldots$

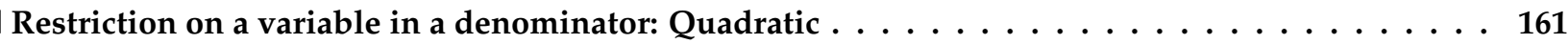

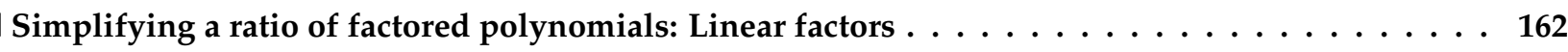

$\square$ Simplifying a ratio of polynomials using GCF factoring . . . . . . . . . . . . . . 163

$\square$ Simplifying a ratio of linear polynomials: $1,-1$, and no simplification . . . . . . . . . 163

$\square$ Simplifying a ratio of polynomials by factoring a quadratic with leading coefficient $1 \ldots 164$

$\square$ Simplifying a ratio of polynomials: Problem type $1 \ldots \ldots \ldots \ldots$

Multiplying rational expressions involving multivariate monomials $\ldots \ldots \ldots$. . . . . . 166

$\square$ Multiplying rational expressions made up of linear expressions $\ldots \ldots \ldots$. . . . . . 167

Dividing rational expressions involving linear expressions $\ldots \ldots \ldots \ldots \ldots$

$\square$ Dividing rational expressions involving quadratics with leading coefficients of $1 \ldots \ldots 8$

$\square$ Introduction to the LCM of two monomials . . . . . . . . . . . . . . . . . . . . . . 169

$\square$ Writing equivalent rational expressions with monomial denominators . . . . . . . . . . 169

$\square$ Finding the LCD of rational expression with linear denominators: Relatively prime . . . . . . . 170

Adding rational expressions with common denominators and GCF factoring . . . . . . . . 171

Adding rational expressions with linear denominators without common factors: Basic . . . . 172

Adding rational expressions with linear denominators with common factors: Basic $\ldots \ldots$

$\square$ Adding rational expressions with denominators $a x^{n}$ and $b x^{m} \ldots \ldots \ldots \ldots \ldots$

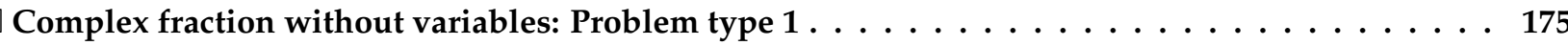

Complex fraction without variables: Problem type $2 \ldots \ldots \ldots \ldots \ldots$

\section{Weekly Checklist}

Complete MALL time.

Work in ALEKS and Notebook at least 3 days a week.

Complete the weekly Module and Notebook pages by the due date.

Attend Focus Group.

Actively participate in Focus Group.

Earn extra credit: Complete 10 topics by 


\section{Evaluating a rational function: Problem type 1}

Open the e-book to complete the following.

\section{Definition of a Rational Function}

A function is a if it can be written in the form Where

$p$ and $q$ are and

$\square$ Watch Video 2: Evaluating a Rational Function for Selected Values in the Domain to complete the following.

Evaluate the function for the given value of $x . \quad f(x)=$

1.

2.

3.

4.

The function is at

The value 3 is in the of the function.

Write the domain of the function in set-builder notation and in interval notation. $f(x)=$ 
EXAMPLE:

Given $f(x)=\frac{x+3}{6-3 x}$, find the following.

a. $f(4)$

$$
f(4)=\frac{4+3}{6-3(4)}=\frac{7}{6-12}=-\frac{7}{6}
$$

b. $f(-5)$

$$
\begin{aligned}
f(-5) & =\frac{-5+3}{6-3(-5)} \\
& =\frac{-2}{6-(-15)} \\
& =\frac{-2}{21}=-\frac{2}{21}
\end{aligned}
$$

c. $f(2)$

$f(2)=\frac{2+3}{6-3(2)}=\frac{5}{0} \Longrightarrow f(2)$ is undefined because the function is not defined at $x=2$.
YOU TRY IT:

Given $g(x)=\frac{7-4 x}{7 x-4}$, find the following.

141. $g(1)$

142. $g\left(\frac{4}{7}\right)$

143. $g(0)$

\section{Restriction on a variable in a denominator: Quadratic}

Division by is

So the expression is undefined when its is

We must find all values of $x$ for which the expression is undefined.

So we set the equal to and solve.

EXAMPLE: Find all excluded values for $\frac{y+2}{y^{2}-9}$.

We must exclude values when $y^{2}-9=0$.

$$
\begin{gathered}
y^{2}-9=0 \\
(y-3)(y+3)=0 \\
y-3=0 \text { or } y+3=0 \\
y=3,-3
\end{gathered}
$$

\section{YOU TRY IT:}

144. Find all excluded values of $\frac{u+7}{u^{2}-4 u+4}$.

$\frac{y+2}{y^{2}-9}$ is undefined when $y=3$ or $y=-3$. 


\section{Simplifying a ratio of factored polynomials: Linear factors}

Watch Video 5: Simplifying a Rational Expression to complete the following. NOTE: This may not be the first video that pops up. Select this video from the list of videos on the left of the video box.

\section{PROPERTY Fundamental Principle of Rational Expressions}

Let $p, q$, and $r$ represent polynomials such that $q \neq 0$ and $r \neq 0$. Then

$$
\frac{p r}{q r}=\longleftarrow=
$$

Simplify the expression.

The expressions $\frac{2 x-6}{x^{2}-8 x+15}$ and $\frac{2}{x-5}$ are equivalent for all real numbers except and because they make the denominator equal to

EXAMPLE: Simplify.

$$
\frac{5(2 x+1)(x-4)}{35(x-4)(x-3)}
$$

Divide the numerator and denominator by 5 .

$$
\frac{5^{1}(2 x+1)(x-4)}{357(x-4)(x-3)}=\frac{(2 x+1)(x-4)}{7(x-4)(x-3)}
$$

Divide the numerator and denominator by $x-4$.

$$
\frac{(2 x+1)(x-4)^{1}}{7(x-4)_{1}(x-3)}=\frac{2 x+1}{7(x-3)}
$$

$\frac{5(2 x+1)(x-4)}{35(x-4)(x-3)}=\frac{2 x+1}{7(x-3)}$ for all real numbers except $x=4$ and $x=3$ because they make the denominator equal to 0 .
YOU TRY IT:

145. Simplify $\frac{8(2 x+3)(x-7)}{18(2 x+3)(x+7)}$. 


\section{Simplifying a ratio of polynomials using GCF factoring}

Learning Page To simplify the expression, we first

the and

the

Then, we look to

\section{EXAMPLE:}

Simplify $\frac{18 x^{2}-24 x}{18 x^{2}-36 x}$

We factor out the greatest common factor (GCF)

from the numerator and the denominator.

$$
\frac{18 x^{2}-24 x}{18 x^{2}-36 x}=\frac{6 x(3 x-4)}{18 x(x-2)}
$$

Divide numerator and denominator by $6 x$.

$$
\frac{6 x^{1}(3 x-4)}{18 x_{3}(x-2)}=\frac{3 x-4}{3(x-2)}
$$

\section{YOU TRY IT:}

146. Simplify $\frac{24 x^{2}+2 x}{12 x^{2}+x}$.

\section{Simplifying a ratio of linear polynomials: $1,-1$, and no simplification}

$\square$ Watch Video 7: Recognizing a ratio of -1 to complete the following.

\section{Recognizing a ratio of 1}

$\frac{5}{5}=$ $=1$

Recognizing a ratio of -1

$$
\frac{5}{-5}=
$$

Additional examples of a ratio $=-1$ : 
EXAMPLE: Simplify.

a. $\frac{x-4}{4-x}$

$$
\begin{aligned}
\frac{x-4}{4-x} & =\frac{x-4}{-1(-4+x)} \\
& =\frac{x-4}{-1(x-4)} \\
& =-1
\end{aligned}
$$

b. $\frac{3 x-6 y}{2 y+x}$

$$
\frac{3 x-6 y}{2 y+x}=\frac{3(x-2 y)}{2 y+x}
$$

Cannot be simplified.
YOU TRY IT: Simplify.

147. $\frac{x+2}{2-x}$

\section{Simplifying a ratio of polynomials by factoring a quadratic with leading coefficient} 1

If you have not completed the Notebook entry for the topic, "Simplifying a ratio of factored polynomials: Linear factors", watch Video 5: Simplifying a Rational Expression and complete the notes now.

EXAMPLE: Simplify $\frac{x^{2}+x-6}{4 x-8}$.

We factor out the GCF from the numerator and factor the denominator.

$$
\begin{aligned}
\frac{x^{2}+x-6}{4 x-8} & =\frac{(x+3)(x-2)}{4(x-2)} \\
& =\frac{x+3}{4}
\end{aligned}
$$

\section{YOU TRY IT:}

149. Simplify $\frac{x^{2}+3 x+2}{3 x+6}$. 


\section{Simplifying a ratio of polynomials: Problem type 1}

$D$ Watch Video 6: Identifying the Restricted valued for a Rational Expression and Simplifying the Expression to complete the following. NOTE: This may not be the first video that pops up. Select this video from the list of videos on the left of the video box.

1. Factor the numerator and denominator.

2. Identify the restricted value(s) for $x$ in this expression.

3. Simplify the expression.

EXAMPLE: Simplify $\frac{x^{2}+x-6}{8-4 x}$.

We factor out the GCF from the numerator and factor the denominator.

$$
\begin{aligned}
\frac{x^{2}+x-6}{8-4 x} & =\frac{(x+3)(x-2)}{4(2-x)} \\
& =\frac{(x+3)(x-2)}{4(-1)(x-2)} \\
& =\frac{(x+3)(x-2)}{-4(x-2)} \\
& =\frac{x+3}{-4} \text { or } \frac{-(x+3)}{4} \text { or }-\frac{x+3}{4}
\end{aligned}
$$

\section{YOU TRY IT:}

150. Simplify $\frac{15-5 x}{x^{2}-9 x+18}$. 


\section{Multiplying rational expressions involving multivariate monomials}

$\square$ Watch Video 1: Multiplying Rational Expressions to complete the following

PROCEDURE To multiply rational expressions, multiply the and

multiply the Then simplify if possible.

$$
\frac{p}{q} \cdot \frac{r}{s}=\ldots \text { provided that } q \neq 0 \text { and } s \neq 0
$$

Multiply the rational expressions.

1.

2.

EXAMPLE: Multiply $\frac{4 m^{4} n^{2}}{3 m n^{5}} \cdot \frac{15 n}{2 m^{2}}$.

$$
\begin{aligned}
\frac{4 m^{4} n^{2}}{3 m n^{5}} \cdot \frac{15 n}{2 m^{2}} & =\frac{2 \cdot 2 \cdot m^{4} \cdot n^{2} \cdot 3 \cdot 5 \cdot n}{3 \cdot m \cdot n^{5} \cdot 2 \cdot m^{2}} \\
& =\frac{2 \cdot 2 \cdot 3 \cdot 5 \cdot m^{4} \cdot n^{3}}{2 \cdot 3 \cdot m^{3} \cdot n^{5}}
\end{aligned}
$$

Divide numerator and denominator by 2 and 3 .

$$
\begin{aligned}
& =\frac{2 \cdot 2 \cdot \not 3 \cdot 5 \cdot m^{4} \cdot n^{3}}{2 \cdot \not 3 \cdot m^{3} \cdot n^{5}} \\
& =\frac{2 \cdot 5 \cdot m^{4} \cdot n^{3}}{m^{3} \cdot n^{5}}
\end{aligned}
$$

Divide numerator and denominator by $n^{3}$.

$$
=\frac{2 \cdot 5 \cdot m^{4}}{m^{3} \cdot n^{2}}
$$

Divide numerator and denominator by $\mathrm{m}^{3}$.

$$
\begin{aligned}
& =\frac{2 \cdot 5 \cdot m}{n^{2}} \\
& =\frac{10 m}{n^{2}}
\end{aligned}
$$


YOU TRY IT:

151. Multiply $\frac{2 a}{3 b^{2}} \cdot \frac{9 b}{14 a^{2}}$.

\section{Multiplying rational expressions made up of linear expressions}

ㅁ․ㅇ Open the e-book to complete the following.

\section{Multiplying Rational Expressions}

Step 1 Factor the and of each expression.

Step 2 the numerators and multiply the

Step 3 Reduce the of factors to 1 or -1 and

EXAMPLE: Multiply $\frac{3 x+6}{5 x-10} \cdot \frac{x-2}{4 x+8}$.

Factor out the GCF in each term then simplify.

$$
\begin{aligned}
\frac{3 x+6}{5 x-10} \cdot \frac{x-2}{4 x+8} & =\frac{3(x+2)}{5(x-2)} \cdot \frac{x-2}{4(x+2)} \\
& =\frac{3(x+2)}{5(x-2)} \cdot \frac{x-2}{4(x+2)} \\
& =\frac{3}{20}
\end{aligned}
$$

\section{YOU TRY IT:}

152. Multiply $\frac{-5 x+15}{2 x+18} \cdot \frac{3 x+27}{x-3}$.

\section{Dividing rational expressions involving linear expressions}

O9pen the e-book to complete the following.

\section{Division Property of Rational Expressions}

Let $p, q, r$, and $s$ represent polynomials, such that $q \neq 0$ and $s \neq 0$. Then

$$
\frac{p}{q} \div \frac{r}{s}=
$$




\section{EXAMPLE:}

Divide $\frac{8 z-16}{-20} \div \frac{3 z-6}{40}$.

$$
\begin{aligned}
\frac{8 z-16}{-20} \div \frac{3 z-6}{40} & =\frac{8 z-16}{-20} \cdot \frac{40}{3 z-6} \\
& =\frac{8(z-2)}{-1 \cdot 20} \cdot \frac{2 \cdot 20}{2(z-2)} \\
& =\frac{8(z-2)}{-1 \cdot 20} \cdot \frac{2 \cdot 20}{2(z-2)} \\
& =-8
\end{aligned}
$$

\section{YOU TRY IT:}

153. Divide $\frac{4 x}{8 x+4} \div \frac{6}{14 x+7}$.

\section{Dividing rational expressions involving quadratics with leading coefficients of 1}

$\square$ Watch Video 4: Dividing Rational Expressions to complete the following.

Divide and simplify.

EXAMPLE:

Divide $\frac{t^{2}-49}{t^{2}+4 t-21} \div \frac{t^{2}-2 t-35}{t^{2}+8 t+15}$

\section{YOU TRY IT:}

154. Divide $\frac{x^{2}-25}{x^{2}+x-20} \div \frac{x^{2}-2 x-15}{x^{2}+7 x+12}$.

$$
\begin{aligned}
\frac{t^{2}-49}{t^{2}+4 t-21} \div \frac{t^{2}-2 t-35}{t^{2}+8 t+15} & =\frac{t^{2}-49}{t^{2}+4 t-21} \cdot \frac{t^{2}+8 t+15}{t^{2}-2 t-35} \\
& =\frac{(t-7)(t+7)}{(t+7)(t-3)} \cdot \frac{(t+3)(t+5)}{(t-7)(t+5)} \\
& =\frac{(t-7)(t+7)}{(t+7)(t-3)} \cdot \frac{(t+3)(t+5)}{(t-7)(t+5)} \\
& =\frac{t+3}{t-3}
\end{aligned}
$$




\section{Introduction to the LCM of two monomials}

?्रि

Open the Instructor Added Resource which will direct you to a video to complete the following.

Find the LCM of and

Multiples of

The LCM of and is since

Multiples of $:$

The LCM of and is since

$\operatorname{LCM}($ )$=$

$\operatorname{LCM}($ )$=$

YOU TRY IT:

155. Find the LCM of $15 x y$ and $20 x^{2} y^{3} z$.

\section{Writing equivalent rational expressions with monomial denominators}

Watch Video 4: Writing Equivalent Fractions to complete the following.

Convert each expression to an equivalent expression with the indicated denominator.

1.

2. 


\section{EXAMPLE:}

Convert each expression to an equivalent expression with the indicated denominator.

$$
\frac{3 x}{5 y^{2}}=\frac{}{20 x y^{5}}
$$

To obtain the fraction equivalent to $\frac{3 x}{5 y^{2}}$ with the denominator $20 x y^{5}$ we multiply by a "form of 1 ".

Since we need to multiply $5 y^{2}$ by $4 x y^{3}$ to get $20 x y^{5}$ our "form of 1 " is

$$
\frac{4 x y^{3}}{4 x y^{3}}
$$

The equivalent fraction is

$$
\frac{3 x}{5 y^{2}} \cdot \frac{4 x y^{3}}{4 x y^{3}}=\frac{12 x^{2} y^{3}}{20 x y^{5}}
$$

YOU TRY IT:

Convert each expression to an equivalent expression with the indicated denominator.

156. $\frac{2 w}{7}=\frac{}{14 w^{5}}$

\section{Finding the LCD of rational expression with linear denominators: Relatively prime}

$\square$ Watch Video 3: Determining the Least Common Denominator Between Two Rational Expressions to complete the following. NOTE: This may not be the first video that pops up. Select this video from the list of videos on the left of the video box.

PROCEDURE Determining the LCD of Two or More Expressions

$\bullet$

- The LCD is the

from the where each factor is raised to its

Determine the LCD for each group of fractions.

1.
2. 
EXAMPLE:

Find the LCD of $\frac{8}{3 x+6}$ and $\frac{x}{4 x-8}$.
YOU TRY IT:

157. Find the LCD of $\frac{5}{6 x-16}$ and $\frac{7}{3 x}$.

We begin by factoring the denominators.

$$
\frac{8}{3 x+6}=\frac{8}{3(x+2)} \quad \frac{x}{4 x-8}=\frac{x}{4(x-2)}
$$

The denominators do not have any common factors. We call these denominators relatively prime.

The LEAST COMMON denominator of relatively prime denominators is the product of all of the factors in the denominators.

The LCD is

$3 \cdot(x+2) \cdot 4 \cdot(x-2)=12(x+2)(x-2)$.

\section{Adding rational expressions with common denominators and GCF factoring}

Open the e-book to complete the following.

\section{Addition and Subtraction of Rational Expressions with Like Denominators}

To add or subtract rational expressions, the expressions must have the

As with fractions, we or rational

expressions with the denominator by the terms in the and then writing the result the Then, if possible, we the expression to

\section{Addition and Subtraction Properties of Rational Expressions}

Let $p, q$, and $r$ represent polynomials where $q \neq 0$. Then

1. $\frac{p}{q}+\frac{r}{q}=$

2. $\frac{p}{q}-\frac{r}{q}=$ 


\section{EXAMPLE:}

Simplify $\frac{x^{2}}{x-4}-\frac{x+12}{x-4}$.

$$
\begin{aligned}
\frac{x^{2}}{x-4}-\frac{x+12}{x-4} & =\frac{x^{2}-(x+12)}{x-4} \\
& =\frac{x^{2}-x-12}{x-4} \\
& =\frac{(x-4)(x+3)}{x-4} \\
& =x+3
\end{aligned}
$$

\section{YOU TRY IT:}

158. Simplify $\frac{6 x}{3 x-1}+\frac{5 x}{3 x-1}$.

Adding rational expressions with linear denominators without common factors: Basic

$\square \quad$ Watch Video 7: Subtracting Rational Expressions to complete the following. NOTE: This video may not pop-up. Select it from the list of videos.

Subtract. 


\section{EXAMPLE:}

Simplify $\frac{3}{x+2}+\frac{5}{x+1}$.

\section{YOU TRY IT:}

159. Simplify $-\frac{2}{x-1}+\frac{3}{x+4}$.

$$
\begin{aligned}
\frac{3}{x+2}+\frac{5}{x+1} & =\frac{3}{x+2} \cdot \frac{x+1}{x+1}+\frac{5}{x+1} \cdot \frac{x+2}{x+2} \\
& =\frac{3(x+1)}{(x+2)(x+1)}+\frac{5(x+2)}{(x+1)(x+2)} \\
& =\frac{3(x+1)+5(x+2)}{(x+1)(x+2)} \\
& =\frac{3 x+3+5 x+10}{(x+1)(x+2)} \\
& =\frac{8 x+13}{(x+1)(x+2)}
\end{aligned}
$$

\section{Adding rational expressions with linear denominators with common factors: Basic}

Learning Page To do the addition, the must be the same.

So first, we find the

Watch the video Exercise: Subtracting Rational Expressions with Unlike Denominators 1 to complete the following.

Add or subtract as indicated. 


\section{Adding rational expressions with denominators $a x^{n}$ and $b x^{m}$}

Open the e-book to complete the following.

\section{Adding and Subtracting Rational Expressions}

Step 1 Factor the of each rational expression.

Step 2 Identify the

Step 3 Rewrite each rational expression as an with the LCD as its denominator.

Step 4 Add or subtract the and write the result over the denominator.

Step 5 Simplify, if possible.

$\square$ Watch Video 5: Adding Rational Expressions to complete the following.

Add.

EXAMPLE: Add.

$$
\frac{6}{7 x}+\frac{4}{x^{3}}
$$

The LCD is $7 x^{3}$

$$
\begin{aligned}
\frac{6}{7 x}+\frac{4}{x^{3}} & =\frac{6}{7 x} \cdot \frac{x^{2}}{x^{2}}+\frac{4}{x^{3}} \cdot \frac{7}{7} \\
& =\frac{6 x^{2}}{7 x^{3}}+\frac{28}{7 x^{3}} \\
& =\frac{6 x^{2}+28}{7 x^{3}}
\end{aligned}
$$

YOU TRY IT: Add.

160. $\frac{5}{6 b^{4}}+\frac{7}{4 b}$ 


\section{Complex fraction without variables: Problem type 1}

$\square$ Watch Video 2: Simplifying a Complex Fraction using Method I to complete the following.

Simplify the complex fraction.

EXAMPLE: Simplify $\frac{\frac{15}{8}}{\frac{5}{2}}$.

A fraction bar means division so we can write the complex fraction as

$$
\frac{\frac{15}{8}}{\frac{5}{2}}=\frac{15}{8} \div \frac{5}{2}
$$

When we divide by a fraction we multiply by the reciprocal of the divisor.

$$
\frac{15}{8} \div \frac{5}{2}=\frac{15}{8} \cdot \frac{2}{5}
$$

Simplifying, $\frac{15^{3}}{8_{4}} \cdot \frac{2^{1}}{\bar{b}_{1}}=\frac{3}{4}$

\section{YOU TRY IT:}

161. Simplify $\frac{\frac{9}{4}}{\frac{3}{10}}$ 


\section{Complex fraction without variables: Problem type 2}

$\square$ Watch Video 2: Simplifying a Complex Fraction using Method I to complete the following.

\section{PROCEDURE Simplifying a Complex Fraction (Method I)}

1. the fractions in the

the fractions in the

2. Divide the by the

3. , if possible.

Simplify the complex fraction by using Method I.

Watch Video 4: Simplifying a Complex Fraction using Method II to complete the following. PROCEDURE Simplifying a Complex Fraction (Method II)

1. and of the complex fraction by the within the expression.

2. Apply the and and

3. , if possible.

\section{Continued on the next page}


Simplify the complex fraction by using Method II.

Notes from Focus Group: 
Module 10

Notes from Focus Group: 


\section{Module 11}

\section{Contents}

$\square$ Square root of a rational perfect square $\ldots \ldots \ldots \ldots \ldots \ldots \ldots \ldots$

$\square$ Square root of a perfect square monomial $\ldots \ldots \ldots \ldots \ldots 18 \ldots \ldots \ldots$

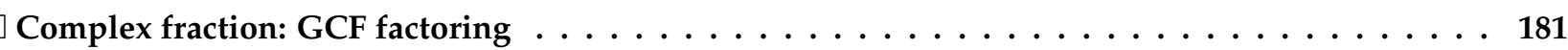

$\square$ Complex fraction made of sums involving rational expressions: Problem type $1 \ldots \ldots 2$

Solving a rational equation that simplifies to linear: Denominators $a, x$, or $a x \ldots \ldots \ldots 3$

$\square$ Solving a rational equation that simplifies to linear: Denominators $a x$ and $b x \ldots \ldots$

$\square$ Solving a rational equation that simplifies to linear: Denominator $x+a \ldots \ldots \ldots$

Solving a rational equation that simplifies to quadratic: Denominator $x \ldots \ldots \ldots$

$\square$ Solving a rational equation that simplifies to quadratic: Proportional form, basic . . . . . . 187

Solving for a variable in terms of other variables in a rational equation: Problem type $1 \ldots \ldots$

$\square$ Solving a proportion of the form $\frac{(x+a)}{b}=\frac{c}{d} \ldots \ldots \ldots \ldots \ldots \ldots$

$\square$ Word problem on proportions: Problem type $1 \ldots \ldots \ldots$. . . . . . . . . . . . . 189

$\square$ Word problem on proportions: Problem type $2 \ldots \ldots \ldots \ldots$

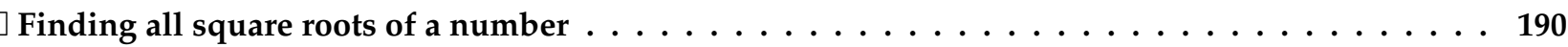

Estimating a square root $\ldots \ldots \ldots \ldots \ldots \ldots \ldots \ldots$

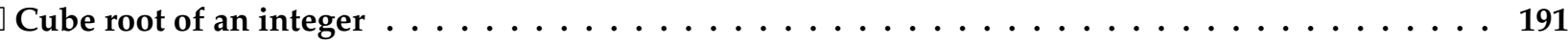

$\square$ Introduction to simplifying a radical expression with an even exponent . . . . . . . . . 192

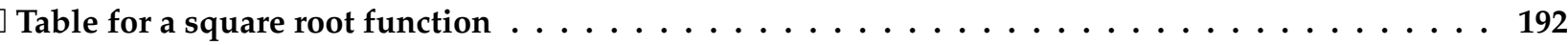

Word problem involving multiple rates $\ldots \ldots \ldots \ldots \ldots \ldots$

$\square$ Graphing a square root function: Problem type $1 \ldots \ldots \ldots \ldots \ldots$

\section{Weekly Checklist}

Complete MALL time.

Work in ALEKS and Notebook at least 3 days a week.

Complete the weekly Module and Notebook pages by the due date.

Attend Focus Group.

Actively participate in Focus Group.

Earn extra credit: Complete 10 topics by 


\section{Square root of a rational perfect square}

$\square$ Watch the video Video 11: Evaluating Square Roots to complete the following.

Finding the of a number is essentially like

Simplify the expressions.

1.

2.

3.

EXAMPLE: Simplify, if possible.

$$
\sqrt{\frac{54}{24}}
$$

54 and 24 are not perfect squares so first try to simplify the fraction in the radical.

$$
\sqrt{\frac{54}{24}}=\sqrt{\frac{9 \cdot 6}{4 \cdot 6}}
$$

We can divide 6 out of the numerator and denominator so that,

$$
\sqrt{\frac{9 \cdot 6}{4 \cdot 6}}=\sqrt{\frac{9}{4}}
$$

Since $\left(\frac{3}{2}\right)^{2}=\frac{9}{4}$
Simplify, if possible.

4.

5.
162. $\sqrt{49}=$

$$
\sqrt{\frac{9}{4}}=\frac{3}{2}
$$

164. $\sqrt{-64}=$ 


\section{Square root of a perfect square monomial}

Learning Page To find this number, we can use the following property of square roots.

$$
\sqrt{A B}=\ldots \text { for any_ numbers } A \text { and } B
$$

Carefully read through the example on the Learning Page.

EXAMPLE: Simplify the following.

$$
\sqrt{81 x^{10}}
$$

We can write

$$
\sqrt{81 x^{10}}=\sqrt{9^{2} \cdot\left(x^{5}\right)^{2}}
$$

Which is equal to $9 x^{5}$. Thus,

$$
\sqrt{81 x^{10}}=9 x^{5}
$$

\section{Complex fraction: GCF factoring}

166. $\sqrt{49 y^{8}}=$

YOU TRY IT: Simplify the following.

165. $\sqrt{a^{14}}=$

167. $\sqrt{16 x^{6}}=$

Watch the video Exercise: Simplifying Complex Fractions Using Method I to complete the following.

Simplify the complex fraction by using Method I.

EXAMPLE: Simplify.

$$
\begin{aligned}
\frac{\frac{3 x}{x-5}}{\frac{7}{4 x-20}} & =\frac{3 x}{x-5} \cdot \frac{4 x-20}{7} \\
& =\frac{3 x}{x-5} \cdot \frac{4(x-5)}{7} \\
& =\frac{3 x}{x-5} \cdot \frac{4(x-5)}{7} \\
& =\frac{12 x}{7}
\end{aligned}
$$

YOU TRY IT: Simplify .

168. $\frac{\frac{7 a}{a+3}}{\frac{1}{4 a+12}}$. 


\section{Complex fraction made of sums involving rational expressions: Problem type 1}

$\square$ Watch Video 3: Simplifying a Complex Fraction Using Method I to complete the following. NOTE: This may not be the first video that pops up. Select this video from the list of videos on the left of the video box.

Simplify the complex fraction by using Method I.

EXAMPLE: Simplify $\frac{1-\frac{4}{3 x}}{\frac{4}{3 x}+1}$.

YOU TRY IT:

Simplify.

Using Method II,

$$
\begin{aligned}
\frac{\left(1-\frac{4}{3 x}\right) \cdot 3 x}{\left(\frac{4}{3 x}+1\right) \cdot 3 x} & =\frac{1 \cdot 3 x-\frac{4}{3 x} \cdot 3 x}{\frac{4}{3 x} \cdot 3 x+1 \cdot 3 x} \\
& =\frac{3 x-4}{4+3 x}
\end{aligned}
$$




\section{Solving a rational equation that simplifies to linear: Denominators $a, x$, or $a x$}

ㅁ․ㅇ Open the e-book to complete the following.

\section{Solving a Rational Equation}

1. Factor the of all rational expressions. Identify any values of the variable for which any expression is

2. Identify the of all terms in the equation.

3. both sides of the equation by the

4. Solve the

5. Check the potential solutions in the original equation. Note that for which the equation is cannot be a solution to the equation.

$\square$ Watch Video 2: Solving a Rational Equation to complete the following.

Solve the equation. 


\section{EXAMPLE:}

Solve $\frac{1}{2}+\frac{9}{x}=\frac{2}{3}$.

$$
\begin{aligned}
\frac{1}{2}+\frac{9}{x} & =\frac{2}{3} \\
\frac{6 x}{1}\left(\frac{1}{2}+\frac{9}{x}\right) & =\frac{6 x}{1} \frac{2}{3} \\
\frac{6 x}{1} \cdot \frac{1}{2}+\frac{6 x}{1} \cdot \frac{9}{x} & =\frac{6 x}{1} \cdot \frac{2}{3} \\
3 x+54 & =4 x \\
54 & =x
\end{aligned}
$$

YOU TRY IT:

170. Solve $\frac{3}{4}-\frac{4}{x}=\frac{5}{6}$.

Solving a rational equation that simplifies to linear: Denominators $a x$ and $b x$

$\square$ Watch the video Exercise: Solving a Rational Equation 1 to complete the following.

Solve the rational equation. 
EXAMPLE:

Solve $\frac{1}{2}-\frac{3}{2 p}=\frac{p-4}{p}$.

$$
\frac{1}{2}-\frac{3}{2 p}=\frac{p-4}{p}
$$

Multiply both sides of the equation by $2 p$

$$
\begin{aligned}
2 p\left(\frac{1}{2}-\frac{3}{2 p}\right) & =2 p\left(\frac{p-4}{p}\right) \\
2 p\left(\frac{1}{2}\right)-2 p\left(\frac{3}{2 p}\right) & =2 \not p\left(\frac{p-4}{\not p}\right) \\
p-3 & =2(p-4) \\
p-3 & =2 p-8 \\
-p & =-5 \\
p & =5
\end{aligned}
$$

YOU TRY IT:

171. Solve $\frac{2}{3 y}+\frac{1}{4}=\frac{11}{6 y}-\frac{1}{3}$.

\section{Solving a rational equation that simplifies to linear: Denominator $x+a$}

$\square$ Watch the video Exercise: Solving a Rational Equation 3 to complete the following. NOTE: This may not be the first video that pops up. Select this video from the list of videos on the left of the video box.

Solve the rational equation.

$x \neq$ 


\section{EXAMPLE:}

Solve $\frac{3}{p-7}=-2$

$$
\frac{3}{p-7}=-2
$$

Multiply both sides of the equation by the LCD $p-7$

$$
\begin{aligned}
(p-7)\left(\frac{3}{p-7}\right) & =(p-7)(-2) \\
(p-7)\left(\frac{3}{p-7}\right) & =(p-7)(-2) \\
3 & =-2 p+14 \\
2 p & =11 \\
p & =\frac{11}{2}
\end{aligned}
$$

YOU TRY IT:

172. Solve $5=\frac{4}{2 y+1}$.

\section{Solving a rational equation that simplifies to quadratic: Denominator $x$}

$\square$ Watch Video 4: Solving a Rational Equation to complete the following.

Solve the equation. 
EXAMPLE: Solve $2+\frac{6}{a}=a+7$.

Multiply both sides of the equation by $a$

$$
\begin{aligned}
a\left(2+\frac{6}{a}\right) & =a(a+7) \\
2 a+6 & =a^{2}+7 a \\
a^{2}+5 a-6 & =0 \\
(a-1)(a+6) & =0 \\
a & =1,-6
\end{aligned}
$$

YOU TRY IT:

173. Solve $\frac{3}{x^{2}}=2+\frac{1}{x}$.

Solving a rational equation that simplifies to quadratic: Proportional form, basic

$\square$ Watch the video Exercise: Solving a Proportion to complete the following.

Solve the proportion.

EXAMPLE: Solve $\frac{1}{b-5}=\frac{b-3}{3}$.

Multiply both sides of the equation by the $\operatorname{LCD} 3(b-5)$.

$$
3(b-5) \cdot \frac{1}{b-5}=3(b-5) \cdot \frac{b-3}{3}
$$

Divide out common factors in the numerators and denominators

$$
\begin{aligned}
3(b-5) \cdot \frac{1}{b-5} & =\not{\not}(b-5) \cdot \frac{b-3}{\not 3} \\
(b-5)(b-3) & =1 \cdot 3 \\
b^{2}-3 b-5 b+15-3 & =0 \\
b^{2}-8 b+12 & =0 \\
(b-6)(b-2) & =0 \\
b & =6 \text { or } 2
\end{aligned}
$$

\section{YOU TRY IT:}

174. Solve $\frac{4}{y+12}=\frac{y}{8 y+11}$. 
Solving for a variable in terms of other variables in a rational equation: Problem type 1

$\square$ Watch Video 6: Solving a Formula for a Different Variable to complete the following.

\section{Solve for $V$.}

Solving a proportion of the form $\frac{(x+a)}{b}=\frac{c}{d}$

$\square$ Watch Video 1: Introduction to Proportions to complete the following.

Solve the proportion.

DEFINITION The ratio of $\mathbf{a}$ to $\mathbf{b}$ can be written as $\frac{a}{b}$ where $b \neq 0$.

An equation that is called a

For example:

$\frac{a}{b}=\ldots$ for $\_$and 
EXAMPLE:

Solve $\frac{3}{x+4}=-\frac{5}{x-1}$ for $x$.

Multiply both sides of the equation by the LCD $(x+4)(x-1)$

$$
\frac{3}{x+4} \cdot(x+4)(x-1)=\frac{-5}{x-1} \cdot(x+4)(x-1)
$$

Divide out common factors in the numerators and denominators

$$
\begin{aligned}
\frac{3}{x+4} \cdot(x+4)(x-1) & =\frac{-5}{x-1} \cdot(x+4)(x-1) \\
3(x-1) & =-5(x+4) \\
3 x-3 & =-5 x-20 \\
8 x & =-17 \\
x & =-\frac{17}{8}
\end{aligned}
$$

\section{Word problem on proportions: Problem type 1}

$\square$ Watch Video 2: An Application of Rational Equations: Solving a Proportion to complete the following.

Franco drove on of gas in his Honda hybrid. How many gallons will he need for a trip across country? 


\section{Word problem on proportions: Problem type 2}

$\square$ Watch Video 3: An Application of Rational Equations: Solving a Proportion to complete the following.

The ratio of female to male students taking algebra is If the total number of students taking the algebra class is how many students are $?$

\section{Finding all square roots of a number}

$\square \quad$ Watch the video Video 1: Definition of a Square Root to complete the following.

$b$ is a square root of $a$ if

Determine the square roots of the given real number.

1.

2.
3. 


\section{Estimating a square root}

Learning Page Complete the following table of square roots.

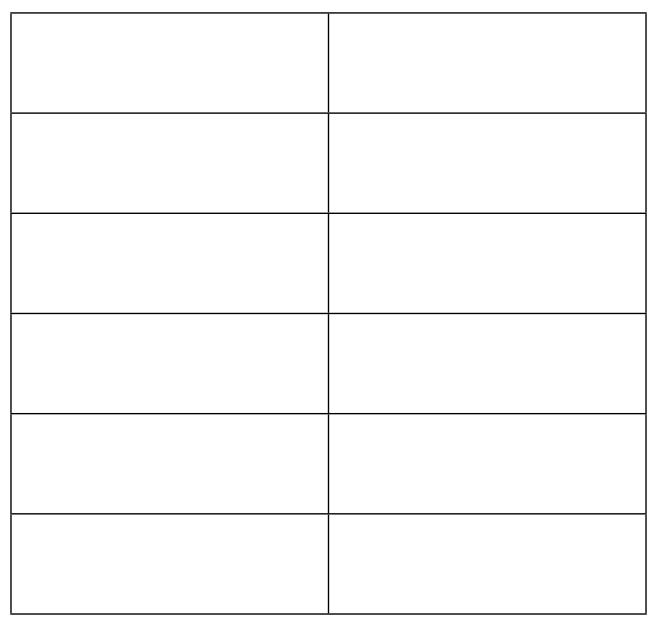

\section{Cube root of an integer}

$\square$ Watch the video Evaluating Roots to complete the following.

YOU TRY IT: Evaluate the roots without using a calculator. Identify those that are not real numbers.

a)

d)

b)

c)

e)

f)

YOU TRY IT: Simplify the following.
176. $\sqrt[3]{125}=$
177. $\sqrt[3]{-8}=$
178. $-\sqrt[3]{216}=$ 


\section{Introduction to simplifying a radical expression with an even exponent}

$\square$ Watch the video Simplifying the $n^{\text {th }}$ Root of Perfect $n^{\text {th }}$ Powers to complete the following.

Simplify the expressions. Assume that all variables represent positive real numbers.

After completing the table to the right II pause the video to try simplifying the expressions on your own.

1.

2.

\begin{tabular}{|ll|}
\hline Perfect squares & Perfect Cubes \\
\hline$\left(x^{1}\right)^{2}=$ & $\left(x^{1}\right)^{3}=$ \\
$\left(x^{2}\right)^{2}=$ & $\left(x^{2}\right)^{3}=$ \\
$\left(x^{3}\right)^{2}=$ & $\left(x^{3}\right)^{3}=$ \\
$\left(x^{4}\right)^{2}=$ & $\left(x^{4}\right)^{3}=$ \\
& \\
\end{tabular}

Continue the video to check your answers.

\section{Table for a square root function}

Learning Page The table gives $x$ and asks that we find the corresponding

Compete the table below from the Learning Page.

\begin{tabular}{|l|l|l|}
\hline$x$ & Evaluate $f(x)=\ldots$ & $f(x)$ \\
\hline & & \\
\hline & & \\
\hline & & \\
\hline & & \\
\hline
\end{tabular}




\section{Word problem involving multiple rates}

$\square$ Watch the video Exercise: Solving a Rational Equation Application Involving Work to complete the following.

Karen can wax her SUV in . Clarann can wax the same SUV in , how long will it take them to wax the SUV together?

\begin{tabular}{|l|l|l|l|}
\hline & Work Rate & Time & $\begin{array}{l}\text { Portion of Job } \\
\text { Completed }\end{array}$ \\
\hline Karen & & & \\
\hline Clarann & & & \\
\hline
\end{tabular}

$(-($

$+$ $=$ 


\section{Graphing a square root function: Problem type 1}

$\square \quad$ Watch the video Video 11: Graphing a Radical Function to complete the following.

Given , the domain is

Graph $f$ by making a table of points.

\begin{tabular}{|l|l|}
\hline$x$ & $f(x)$ \\
\hline & \\
\hline & \\
\hline & \\
\hline & \\
\hline & \\
\hline & \\
\hline
\end{tabular}

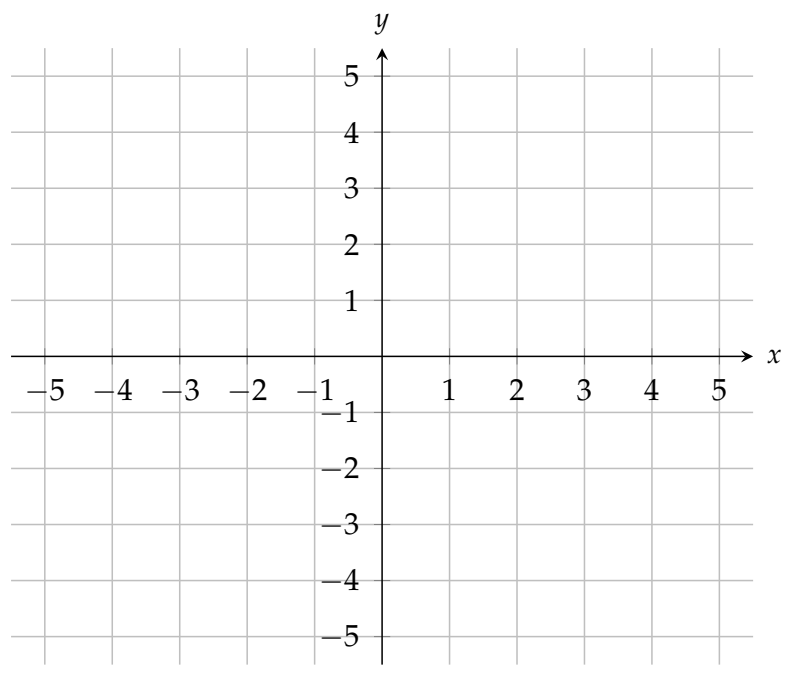

Notes from Focus Group: 
Notes from Focus Group: 


\section{Module 12-Review}

To help you review for your upcoming exam, this module contains all of the topics from the modules since the last exam. Topics that you have already mastered will not appear in your carousel.

Complete this module before you take the ALEKS exam.

Each exam has two parts.

- The ALEKS exam (100 pts)

- The ALEKS exam must be taken in the MALL.

- The ALEKS exam is a Comprehensive Knowledge Check.

- Your score is the number of topics you have mastered out of the number of topics you should have mastered by this point.

- If you lose topics on your ALEKS exam, your Review Module completion grade will not change.

- Your scratch work for the ALEKS exam must be numbered and turned in through Blackboard.

- The Written exam (25 pts)

- Take your written exam in class the day of your focus group.

○ To study for the written exam:

- Rework your old Focus Group assignments.

- Rework any topics in ALEKS you may have lost on the ALEKS exam.

\begin{tabular}{|l|l|}
\hline & Score \\
\hline ALEKS Exam & \\
\hline Written Exam & \\
\hline
\end{tabular}




\section{Module 13}

\section{Contents}

$\square$ Finding $n^{\text {th }}$ roots of perfect $n^{\text {th }}$ powers with signs $\ldots \ldots \ldots \ldots \ldots \ldots$

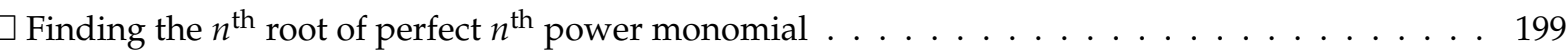

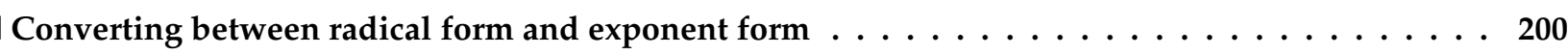

$\square$ Rational exponents: Unit fraction exponents and whole number bases . . . . . . . . . . . . 201

Rational exponents: Unit fraction exponents and bases involving signs . . . . . . . . . . . . . 201

$\square$ Rational exponents: Non-unit fraction exponent with a whole number base . . . . . . . . . . 202

$\square$ Simplifying the square root of a whole number less than $100 \ldots \ldots \ldots$

$\square$ Simplifying the square root of a whole number greater than $100 \ldots \ldots \ldots \ldots$

Rational exponents: Product rule . . . . . . . . . . . . . . . . . . . . . 204

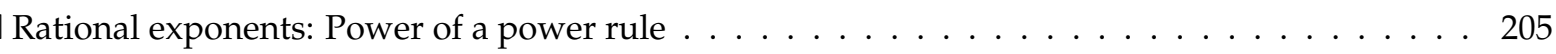

$\square$ Simplifying a radical expression with an even exponent $\ldots \ldots \ldots \ldots \ldots$

$\square$ Simplifying a radical expression with an odd exponent . . . . . . . . . . . . . . 206

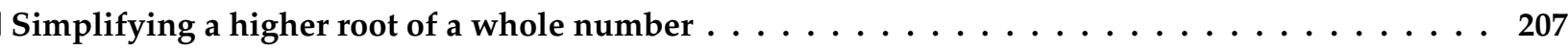

$\square$ Introduction to square root addition or subtraction $\ldots \ldots \ldots \ldots \ldots$

$\square$ Square root addition or subtraction $\ldots \ldots \ldots \ldots \ldots \ldots$

$\square$ Introduction to simplifying a sum or difference of radical expressions: Univariate . . . . . . . 209

$\square$ Simplifying a product involving square roots using the distributive property: Basic . . . . . . 209

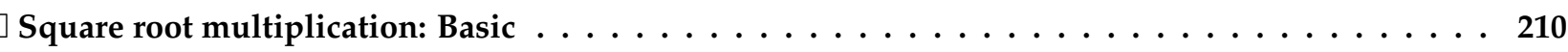

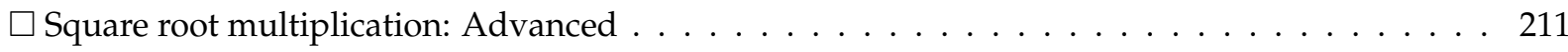

Simplifying a product of radical expressions: Univariate . . . . . . . . . . . 212

\section{Weekly Checklist}

Complete MALL time.

Work in ALEKS and Notebook at least 3 days a week.

Complete the weekly Module and Notebook pages by the due date.

Attend Focus Group.

Actively participate in Focus Group.

Earn extra credit: Complete 10 topics by 


\section{Finding $n^{\text {th }}$ roots of perfect $n^{\text {th }}$ powers with signs}

O․ Open the e-book to complete the following.

\section{Definition of an $n^{\text {th }}$ Root}

$b$ is an $n$th root of a if

Example: 2 is a root of ___ because

Example: 2 is a root of because

Example: 2 is a root of because

\section{Evaluating $\sqrt[n]{a}$}

1. If is an integer and then is the principal (_ $) n$th root of $a$.

Example:

2. If is an integer then is the

\section{Example:}

3. If is an , then

\section{EXAMPLE:}

Evaluate the following.

$$
-\sqrt[4]{81}
$$

Since $3^{4}=81$

\section{YOU TRY IT:}

Simplify the following.

179. $\sqrt[4]{-16}=$

180. $\sqrt[3]{-125}=$

$$
-\sqrt[4]{81}=-\sqrt[4]{3^{4}}=-3
$$




\section{Finding the $n^{\text {th }}$ root of perfect $n^{\text {th }}$ power monomial}

$\square$ Watch the video Simplifying the $n^{\text {th }}$ Root of Perfect $n^{\text {th }}$ Powers to complete the following.

Simplify the expressions. Assume that all variables represent positive real numbers.

After completing the table to the right II pause the video to try simplifying the expressions on your own.

1.

2.

3.

4.

\begin{tabular}{|ll|}
\hline Perfect squares & Perfect Cubes \\
\hline$\left(x^{1}\right)^{2}=$ & $\left(x^{1}\right)^{3}=$ \\
$\left(x^{2}\right)^{2}=$ & $\left(x^{2}\right)^{3}=$ \\
$\left(x^{3}\right)^{2}=$ & $\left(x^{3}\right)^{3}=$ \\
$\left(x^{4}\right)^{2}=$ & $\left(x^{4}\right)^{3}=$ \\
& \\
\end{tabular}

Continue the video to check your answers.

EXAMPLE: Simplify the following.

$$
\sqrt[4]{625 p^{12}}
$$

Since we are finding the $4^{\text {th }}$ root we want to write each factor as an equivalent power of 4 .

We can write $625=5^{4}$ and $p^{12}=\left(p^{3}\right)^{4}$.

$$
\sqrt[4]{625 p^{12}}=\sqrt[4]{5^{4}\left(p^{3}\right)^{4}}=5 p^{3}
$$

\section{YOU TRY IT:}

Simplify the following.

181. $\sqrt[3]{27 x^{15}}=$

182. $\sqrt[5]{32 x^{10}}=$ 


\section{Converting between radical form and exponent form}

$\square$ Watch the video Video 3: Converting Between Radical Notation and Rational Exponents to complete the following.

Convert each expression to radical notation. Assume all variables represent positive real numbers.

1.

6.

YOU TRY IT: Write as an exponential expression.

183. $\sqrt[5]{x^{2}}=$

Write as a radical expression.

185. $x^{5 / 3}=$ real numbers.
4.

6.
2.

3.

Convert each expression to an expression with rational exponents. Assume all variables represent positive

5.

7.

184. $\sqrt{y^{3}}=$

186. $y^{2 / 7}=$ 


\section{Rational exponents: Unit fraction exponents and whole number bases}

Watch the video Definition of " $a$ " to the $1 / n$ Power to complete the following.

DEFINITION Let $a$ be a real number, and let $n>1$ be an integer. Then,

$$
a^{1 / n}=\ldots \text { provided that __ is a real number. }
$$

Write each expression in radical notation and simplify.

1.

4.
2.

5.
3.

6.

\section{Rational exponents: Unit fraction exponents and bases involving signs}

If you have not already done so, watch the video Definition of " $a$ " to the $1 / n$ Power and take notes under the previous topic Rational exponents: Unit fraction exponents and whole number bases.

YOU TRY IT: Simplify the following.
187. $-81^{1 / 4}=$
188. $(-64)^{1 / 3}=$ 


\section{Rational exponents: Non-unit fraction exponent with a whole number base}

$D$ Watch Video 2: Definition of " $a$ " to the $m / n$ Power to complete the following.

DEFINITION Let $a$ be a real number, and let $m$ and $n$ be positive integers that share no common factors other than 1 . Then,

1. $a^{1 / n}=$

2. $a^{m / n}=$ provided that

provided that is real number. is a real number.

Write the expression in radical notation and simplify.

1.

3.

YOU TRY IT: Simplify the following.

189. $27^{2 / 3}=$
2.

4.

190. $(-81)^{3 / 4}=$ 


\section{Simplifying the square root of a whole number less than 100}

$\square$ Watch Video 5: Simplifying a Radical with a Numerical Factor in the Radicand to complete the following.

Simplify the expression

Another way to simplify this is to find the largest perfect square that is a factor of our radicand.

\section{Simplifying the square root of a whole number greater than 100}

Learning Page One of the properties of square roots is the product property.

$$
\sqrt{a \times b}=\ldots \text { for any nonnegative numbers } a \text { and } b
$$

$\square$ If you have not already done so, watch Video 5: Simplifying a Radical with a Numerical Factor in the Radicand and take notes under the previous topic. 


\section{Rational exponents: Product rule}

$\triangleright \quad$ Watch Video 4: Properties of Rational Exponents to complete the following.

PROPERTY Let $a$ and $b$ be nonzero real numbers. Let $m$ and $n$ be rational numbers such that $a^{m}, a^{n}$, and $b^{m}$ are real numbers.

\begin{tabular}{|l|l|l|}
\hline Description & Property & Example \\
\hline Multiplying like bases & $a^{m} a^{n}=\ldots$ & \\
\hline Dividing like bases & $\frac{a^{m}}{a^{n}}=\ldots$ & $\frac{5^{3 / 4}}{5^{1 / 2}}=$ \\
\hline Power rule & $\left(a^{m}\right)^{n}=\ldots$ & \\
\hline Power of a Product & $(a b)^{m}=\ldots$ & $\left(c^{1 / 3} d^{1 / 2}\right)^{6}=$ \\
\hline Power of a Quotient & $\left(\frac{a}{b}\right)^{m}=\ldots$ & \\
\hline
\end{tabular}

Assume that all variables represent positive real numbers.

\section{EXAMPLE:}

Use only positive exponents in your answer.

Simplify $u^{\frac{1}{4}} \cdot u^{-\frac{1}{6}}$.

$$
\begin{aligned}
u^{\frac{1}{4}} \cdot u^{-\frac{1}{6}} & =u^{\frac{1}{4}+\left(-\frac{1}{6}\right)} \quad \text { product rule } \\
& =u^{\frac{3}{12}-\frac{2}{12}} \text { get common denominator } \\
& =u^{\frac{1}{12}}
\end{aligned}
$$

YOU TRY IT:

Simplify. Use only positive exponents in your answer.

191. $x^{-1} \cdot x^{\frac{1}{5}}$ 


\section{Rational exponents: Power of a power rule}

If you have not watched Video 4: Properties of Rational Exponents under the previous topic Rational exponents: Product rule, do so now.

Simplify. Assume that all variables represent positive real numbers and use only positive exponents in your answer.

EXAMPLE: $\left(u^{\frac{1}{4}}\right)^{5}$

YOU TRY IT:

$$
\begin{aligned}
\left(u^{\frac{1}{4}}\right)^{5} & =u^{\frac{1}{4} \cdot 5} \text { power of a power rule } \\
& =u^{\frac{5}{4}} \text { Simplify }
\end{aligned}
$$

\section{Simplifying a radical expression with an even exponent}

Watch Video 7: Simplifying Radicals by using an Alternative Method to complete the following. NOTE: This may not be the first video that pops up. Select this video from the list of videos on the left of the video box.

Simplify the expressions. Assume that $x, y$ and $z$ represent positive real numbers.

1.

2.

Assume that all variables represent positive real numbers.

\section{EXAMPLE:}

Simplify $\sqrt{75 y^{16}}$.

$$
\begin{aligned}
\sqrt{75 y^{16}} & =\sqrt{5^{2} \cdot 3 y^{16}} \text { because } 75=25 \cdot 3 \\
& =5 \sqrt{3 y^{16}} \text { because } \sqrt{25}=5 \\
& =5 y^{8} \sqrt{3} \text { the power divided by the index } \\
& \text { is } 8 \text { with no remainder }
\end{aligned}
$$

YOU TRY IT: Simplify the following.

193. $\sqrt{24 x^{8}}=$

194. $\sqrt{18 x^{10}}=$ 


\section{Simplifying a radical expression with an odd exponent}

$\square$ Watch Video 3: Simplifying Radicals to complete the following. NOTE: This may not be the first video that pops up. Select this video from the list of videos on the left of the video box.

Simplify the expressions. Assume that $x$ and $y$ are positive real numbers.

a)

d) b)

e)

Recall: must be raised to a

Assume that all variables represent positive real numbers.

EXAMPLE:

Simplify $\sqrt{50 p^{11}}$.

$$
\begin{aligned}
\sqrt{50 p^{11}} & =\sqrt{5^{2} \cdot 2 \cdot p^{11}} \text { because } 50=25 \cdot 2 \\
& =5 \sqrt{2 p^{11}} \text { because } \sqrt{5^{2}}=5 \\
& =5 p^{5} \sqrt{2 p} \text { the power divided by the index } \\
& \text { is } 5 \text { with remainder of } 1
\end{aligned}
$$

YOU TRY IT: Simplify the following.

195. $\sqrt{20 x^{11}}=$

196. $\sqrt{27 x^{15}}$ 


\section{Simplifying a higher root of a whole number}

Open the e-book to complete the following.

\section{Simplified Form of a Radical}

Consider any radical expression where the radicand is written as a product of prime factors. The expression is in simplified form if all the following conditions are met:

1. The radicand has raised to a power greater than or equal to the

2. The radicand does not contain a

3. There are no in the of a fraction.

Assume that all variables represent positive real numbers.

EXAMPLE: Simplify $\sqrt[4]{243}$.

YOU TRY IT: Simplify the following.

We can write $243=3^{5}=3^{4} \cdot 3$

$$
\begin{aligned}
\sqrt[4]{243} & =\sqrt[4]{3^{4} \cdot 3} \\
& =\sqrt[4]{3^{4}} \cdot \sqrt[4]{3} \\
& =3 \sqrt[4]{3}
\end{aligned}
$$

\section{Introduction to square root addition or subtraction}

$\square$ Watch the video Adding or Subtracting Radical Expressions 1 to complete the following.

Add or subtract the radical expression, if possible. 
YOU TRY IT: Simplify the following.

198. $7 \sqrt{5}-4 \sqrt{5}=$

199. $8 \sqrt{3}+2 \sqrt{3}=$

\section{Square root addition or subtraction}

Oy Open the e-book to complete the following.

\section{Definition of Like Radicals}

Two radical terms are called like radicals if they have the and

\section{Avoiding Mistakes}

The process of adding like radicals with the distributive property is similar to adding

The end result is that the

are added and the radical factor is

$$
\sqrt{3}+\sqrt{3}=1 \sqrt{3}+1 \sqrt{3}=
$$

Be careful: True or False: $\sqrt{3}+\sqrt{3}=\sqrt{6}$

True or False: $\sqrt{x}+\sqrt{y}=\sqrt{x+y}$

EXAMPLE: Simplify $3 \sqrt{12}+2 \sqrt{48}$.

$$
\begin{aligned}
3 \sqrt{12} & +2 \sqrt{48} \\
& =3 \sqrt{4 \cdot 3}+2 \sqrt{16 \cdot 3} \quad \begin{array}{l}
\text { Factor out the perfect } \\
\text { square in each term }
\end{array} \\
& =3 \cdot 2 \sqrt{3}+2 \cdot 4 \sqrt{3} \quad \begin{array}{c}
\text { Take the square root of } \\
\text { each perfect square }
\end{array} \\
& =6 \sqrt{3}+8 \sqrt{3} \quad \text { Simplify } \\
& =(6+8) \sqrt{3} \quad \text { Distributive property } \\
& =14 \sqrt{3}
\end{aligned}
$$$$
\text { square in each term }
$$$$
\text { each perfect square }
$$

YOU TRY IT: Simplify the following.

200. $3 \sqrt{40}-\sqrt{8}+2 \sqrt{50}=$ 


\section{Introduction to simplifying a sum or difference of radical expressions: Univariate}

Watch Video 3: Adding and Subtracting Radicals to complete the following.

Add or subtract as indicated.

EXAMPLE:

Simplify $\sqrt{24 x}-\sqrt{54 x}$

$\sqrt{24 x}-\sqrt{54 x}$

$=\sqrt{4 \cdot 6 x}-\sqrt{9 \cdot 6 x}$ Factor out the perfect square in each term

$=2 \sqrt{6 x}-3 \sqrt{6 x}$ Take the square root of each perfect square

$=(2-3) \sqrt{6 x}$ Distributive property

$=-\sqrt{6 x}$
YOU TRY IT: Simplify the following.

201. $\sqrt{75 w}-\sqrt{27 w}=$

\section{Simplifying a product involving square roots using the distributive property: Basic}

$\square$ Watch the video Multiplying Radical Expressions 2 to complete the following.

Multiply the radical expressions. 
EXAMPLE: Multiply $3 \sqrt{5}(2 \sqrt{5}+4)$

$$
\begin{aligned}
& 3 \sqrt{5}(2 \sqrt{5}+4) \\
& =3 \sqrt{5} \cdot 2 \sqrt{5}+3 \sqrt{5} \cdot 4 \quad \text { Use the distributive } \\
& \text { property } \\
& =3 \cdot 2 \sqrt{5} \cdot \sqrt{5}+4 \cdot 3 \sqrt{5} \quad \begin{array}{c}
\text { Commutative property } \\
\text { of multiplication }
\end{array} \\
& =6 \sqrt{5 \cdot 5}+12 \sqrt{5} \quad \text { Use the property } \\
& =6 \cdot 5+12 \sqrt{5} \quad \text { Because } \sqrt{5 \cdot 5}=5 \\
& =30+12 \sqrt{5}
\end{aligned}
$$

YOU TRY IT: Simplify the following.

202. $2 \sqrt{6}(\sqrt{3}-\sqrt{7})=$

\section{Square root multiplication: Basic}

$D$ Watch Video 1: Introduction to the Multiplication of Radicals to complete the following.

\section{PROPERTY Multiplication Property of Radicals}

Let $a$ and $b$ represent real numbers such that $\sqrt[n]{a}$ and $\sqrt[n]{b}$ are real numbers.

$$
\sqrt[n]{a} \cdot \sqrt[n]{b}=
$$

Multiply. Assume that $x$ represents a positive real number.

1.

2.

3. 


\section{Square root multiplication: Advanced}

$\triangleright$ Watch Video 2: Multiplying Radical Expressions to complete the following.

Multiply and simplify the result. Assume that all variables represent positive real numbers.

1.

2.

EXAMPLE: Simplify $2 \sqrt{20} \cdot \sqrt{54}$

$2 \sqrt{20} \cdot \sqrt{54}$

$=2 \sqrt{20 \cdot 54}$ Use the property $\sqrt{A} \sqrt{B}=\sqrt{A B}$

for positive $A$ and $B$

$=2 \sqrt{4 \cdot 5 \cdot 9 \cdot 6}$ Factor out the perfect squares

$=2 \sqrt{4 \cdot 9 \cdot 5 \cdot 6}$

$=2 \cdot 2 \cdot 3 \sqrt{30}$ Take the square root of the

perfect squares

$=12 \sqrt{30}$
YOU TRY IT: Simplify the following.

203. $3 \sqrt{24} \cdot 2 \sqrt{18}=$ 


\section{Simplifying a product of radical expressions: Univariate}

Learning Page Take notes from the Learning Page.

Simplify Assume that all variables represent positive real numbers.

$$
=
$$

$$
=
$$$$
=
$$$$
=
$$$$
=
$$$$
=
$$

EXAMPLE: Simplify $\sqrt{5 b} \cdot \sqrt{15 b^{2}}$

$$
\begin{aligned}
& \sqrt{5 b} \cdot \sqrt{15 b^{2}} \\
& =\sqrt{5 b \cdot 15 b^{2}} \text { Use the property } \sqrt{A} \sqrt{B}=\sqrt{A B} \\
& \text { for positive } A \text { and } B \\
& =\sqrt{75 b^{3}} \quad \text { Multiply under the square root sign } \\
& =\sqrt{25 b^{2} \cdot 5 b} \text { Factor out the perfect square } \\
& =5 b \sqrt{5 b} \quad \text { Take the square root of the } \\
& \text { perfect square }
\end{aligned}
$$

YOU TRY IT: Simplify the following.

204. $\sqrt{12 x^{5}} \cdot 2 \sqrt{10 x}=$

\section{Notes from Focus Group:}


Notes from Focus Group: 


\section{Module 14}

\section{Contents}

$\square$ Simplifying a quotient of square roots $\ldots \ldots \ldots \ldots \ldots \ldots \ldots \ldots$

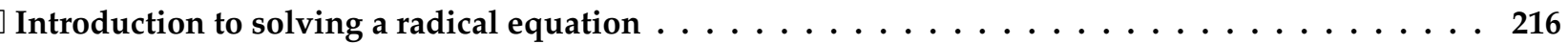

$\square$ Solving a radical equation that simplifies to a linear equation: One radical, basic . . . . . 217

$\square$ Solving a radical equation that simplifies to a linear equation: One radical, advanced . . . . . 218

$\square$ Solving a radical equation that simplifies to a quadratic equation: One radical, basic . . . . . 219

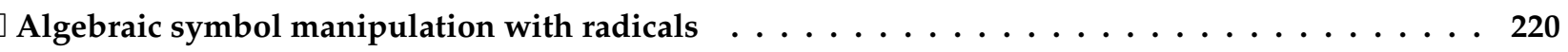

$\square$ Word problem involving radical equations: Advanced . . . . . . . . . . . . . . . 221

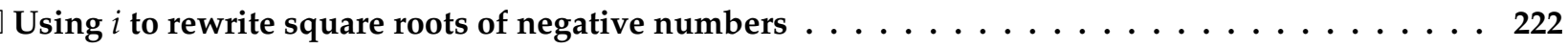

$\square$ Solving an equation of the form $x^{2}=a$ using the square root property $\ldots \ldots \ldots 22$

$\square$ Solving a quadratic equation using the square root property: Exact answers, basic . . . . . . . 223

$\square$ Solving a quadratic equation using the square root property: Exact answers, advanced . . . . 224

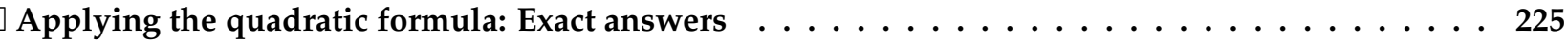

$\square$ Solving a quadratic equation with complex roots $\ldots \ldots \ldots \ldots \ldots \ldots \ldots \ldots$

Domain and range from the graph of a parabola $\ldots \ldots \ldots \ldots \ldots \ldots \ldots \ldots \ldots \ldots \ldots$

How the leading coefficient affects the shape of the parabola $\ldots \ldots \ldots \ldots \ldots \ldots$

$\square$ Finding the vertex, intercepts, and axis of symmetry from the graph of a parabola . . . . . . . 229

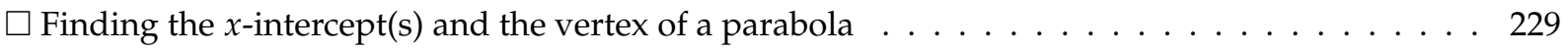

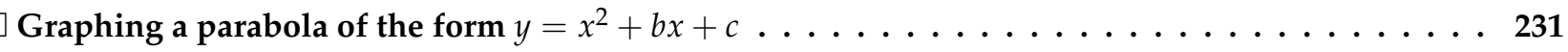

$\square$ Graphing a parabola of the form $y=a x^{2}+b x+c$ : Integer coefficients $\ldots \ldots \ldots 232$

\section{Weekly Checklist}

Complete MALL time.

Work in ALEKS and Notebook at least 3 days a week.

Complete the weekly Module and Notebook pages by the due date.

Attend Focus Group.

Actively participate in Focus Group.

Earn extra credit: Complete 10 topics by 


\section{Simplifying a quotient of square roots}

Learning Page We'll use the following property of square roots to simplify our expression.

\section{Quotient property of square roots}

$=$ for any nonnegative numbers $a$ and $b$, where $b \neq 0$

Carefully read through the example on the Learning Page and take notes.

EXAMPLE: Simplify $\sqrt{\frac{2}{10}}$

$\sqrt{\frac{2}{10}}=\sqrt{\frac{1}{5}} \quad$ Divide under the square root sign $=\frac{\sqrt{1}}{\sqrt{5}} \quad$ Use quotient property of square roots

$=\frac{1}{\sqrt{5}} \quad$ Simplify the square root: $\sqrt{1}=1$
YOU TRY IT: Simplify the following.

205. $\sqrt{\frac{9}{7}}=$

A simplified radical fraction does NOT have a radical in the denominator.

We rationalize the denominator by multiplying by $\frac{\sqrt{5}}{\sqrt{5}}$, which equals 1 .

$$
\frac{1}{\sqrt{5}} \cdot \frac{\sqrt{5}}{\sqrt{5}}=\frac{\sqrt{5}}{5} \Longrightarrow \sqrt{\frac{2}{10}}=\frac{\sqrt{5}}{5}
$$




\section{Introduction to solving a radical equation}

$\square$ Watch Video 1: Introduction to Radical Equations to complete the following.

Solve the equations.

1.

2.

\section{Avoiding Mistakes}

When an equation is raised to an , it is necessary to

in the original equation.

Show why this is important.

YOU TRY IT: Solve the following.

206. $\sqrt{y}=-7$

207. $\sqrt{x}=8$ 


\section{Solving a radical equation that simplifies to a linear equation: One radical, basic}

$\square \quad$ Watch Video 2: Procedure to Solve a Radical Equation to complete the following.

\section{PROCEDURE Solving Radical Equations}

1. If an equation has more than one radical, choose one of the radicals to isolate.

2.

3. If the equation still has a radical, repeat steps 1 and 2.

4.

Solve the equation.

Watch Video 4: Solving a Radical Equation with No Solution to complete the following. NOTE: This may not be the first video that pops up. Select this video from the list of videos on the left of the video box.

Solve 
Solving a radical equation that simplifies to a linear equation: One radical, advanced

$\square$ Watch the video Exercise: Solving an Equation Containing One Radical 1 to complete the following. NOTE: This may not be the first video that pops up. Select this video from the list of videos on the left of the video box.

Solve the equation.

EXAMPLE:

Solve $\sqrt{y+8}+2=4$

$$
\begin{aligned}
\sqrt{y+8}+2 & =4 \\
\sqrt{y+8} & =2 \\
(\sqrt{y+8})^{2} & =(2)^{2} \\
y+8 & =4 \\
y & =-4
\end{aligned}
$$

Check the solution.

$$
\begin{aligned}
\sqrt{-4+8}+2 & \stackrel{?}{=} 4 \\
\sqrt{4}+2 & \stackrel{?}{=} 4 \\
4 & =4
\end{aligned}
$$

YOU TRY IT:

208. Solve $\sqrt{2 x+29}+3=1$

$y=-4$ is a solution. 


\section{Solving a radical equation that simplifies to a quadratic equation: One radical, basic}

$\square$ Watch the video Solving a Radical Equation in which One Potential Solution Does not Check to complete the following.

Solve the equation.

EXAMPLE: Solve for $y$.

$$
\begin{aligned}
\sqrt{y+18}+2 & =y \\
\sqrt{y+18} & =y-2 \\
(\sqrt{y+18})^{2} & =(y-2)^{2} \\
y+18 & =y^{2}-4 y+4 \\
0 & =y^{2}-5 y-14 \\
0 & =(y-7)(y+2) \\
y & =-2,7
\end{aligned}
$$

YOU TRY IT: Solve for $x$.

209. $\sqrt{2 x+29}+3=x$

Check the solutions.

$$
\begin{array}{rlrl}
\sqrt{-2+18}+2 & \stackrel{?}{=}-2 & \sqrt{7+18}+2 \stackrel{?}{=} 7 \\
\sqrt{16}+2 \stackrel{?}{=}-2 & \sqrt{25}+2 \stackrel{?}{=} 7 \\
4+2 \stackrel{?}{=}-2 & 5+2 \stackrel{?}{=} 7 \\
6 & \neq-2 & 7 & =7
\end{array}
$$

$y=7$ is a solution. 


\section{Algebraic symbol manipulation with radicals}

$\square \quad$ Watch the video Exercise: Solving an Equation Containing One Radical 3 to complete the following.

Assume all variables represent positive real numbers.

Solve for

YOU TRY IT: Solve for $V$.

210. $b=\sqrt{\frac{3 V}{h}}$ 


\section{Word problem involving radical equations: Advanced}

$\square \quad$ Watch the video Exercise: Solving an Equation Containing One Radical 7 to complete the following.

If an object is dropped from an its velocity at impact with the ground is given by where $g$ is the acceleration due to gravity and $h$ is the initial height.

a. Find the initial height (in feet) of an object if its $\mathrm{ft} / \mathrm{sec}$.

(Assume that the acceleration due to gravity is -)

b. Find the initial height (in meters) of an object if its $\mathrm{m} / \mathrm{sec}$.

(Assume that the acceleration due to gravity is Round to the nearest tenth of a meter.) 


\section{Using $i$ to rewrite square roots of negative numbers}

Open the e-book to complete the following.

\section{Definition of the Imaginary Number $i$}

$$
i=
$$

Note: From the definition of $i$, it follows that

Definition of $\sqrt{-b}$ for $b>0$

Let $b$ be a positive real number. Then

EXAMPLE: Simplify $\sqrt{-50}$.

We use the letter $i$ for $\sqrt{-1}$.

For any positive real number $a$, we have the property

$$
\begin{array}{r}
\sqrt{-a}=i \cdot \sqrt{a} \\
\sqrt{-50}=i \sqrt{25 \cdot 2}=5 i \cdot \sqrt{2}
\end{array}
$$

YOU TRY IT: Simplify.

211. $\sqrt{-24}$

212. $\sqrt{-15}$

\section{Solving an equation of the form $x^{2}=a$ using the square root property}

$\square$ Watch Video 1: Introduction to the Square Root Property to complete the following.

Solve the equation. This video shows two ways to solve the equation. Make sure to write down BOTH ways.

PROPERTY The Square Root Property

For any real number $k$, if $x^{2}=k$, then 
All variables represent real numbers.

\section{EXAMPLE:}

Solve $x^{2}=-100$.

This equation has no real number solution.

There is no value for $x$ that can be squared to get a negative answer.
YOU TRY IT: Solve.

213. $x^{2}=36$

214. $x^{2}=5$

\section{Solving a quadratic equation using the square root property: Exact answers, basic}

$\square \quad$ Watch Video 2: Solving Quadratic Equations Using the Square Root Property to complete the following.

Solve the equations.

1.

3.
2.

4. 
YOU TRY IT: Solve.

215. $x^{2}-40=0$
216. $3 x^{2}+6=0$

Solving a quadratic equation using the square root property: Exact answers, advanced

$\square$ Watch Video 3: Solving Quadratic Equations Using the Square Root Property to complete the following.

Solve the equations.

1.

2. 
EXAMPLE: Solve: $2(x+1)^{2}-16=0$.

We need to write the equation with only the squared expression on the left side.

$$
2(x+1)^{2}-16=0
$$

Add 16 to both sides

$$
2(x+1)^{2}=16
$$

Divide both sides by 2

$$
(x+1)^{2}=8
$$

Use the Square root property

$$
x+1= \pm \sqrt{8}
$$

Isolate $x$

$$
x=-1 \pm 2 \sqrt{2}
$$

YOU TRY IT:

217. Solve: $\frac{1}{2}(x-2)^{2}-5=0$

\section{Applying the quadratic formula: Exact answers}

$\square$ Watch Video 1: Introduction to the Quadratic Formula to complete the following.

\section{FORMULA The Quadratic Formula}

Given a quadratic equation $a x^{2}+b x+c=0(a \neq 0)$, the solutions are:

$$
x=
$$

Solve the equation by using the Quadratic Formula. 
YOU TRY IT: Solve using the Quadratic Formula.

218. $x^{2}-3 x+1=0$

\section{Solving a quadratic equation with complex roots}

$\square$ Watch the video Solving a Quadratic Equation by Using the Quadratic Formula to complete the following.

Solve the equation by using the quadratic formula. 
EXAMPLE: Solve $5 x^{2}-4 x+1=0$ using the quadratic formula.

$$
\begin{aligned}
5 x^{2}-4 x+1 & =0 \\
x & =\frac{-(-4) \pm \sqrt{(-4)^{2}-4(5)(1)}}{2(5)} \\
x & =\frac{4 \pm \sqrt{-4}}{10} \\
x & =\frac{4 \pm 2 i}{10} \\
x & =\frac{2}{5} \pm \frac{1}{5} i
\end{aligned}
$$

\section{YOU TRY IT:}

219. Solve $3 x^{2}+2 x+1=0$ by using the quadratic formula.

\section{Domain and range from the graph of a parabola}

Learning Page It is possible to determine the domain and range of a function from its graph.

The is the set of all the numbers that appear as of points on the graph.

The is the set of all the numbers that appear as of points on the graph.

The graph of a function is a

When a parabola is a it extends to the left and right It also

extends upward or downward

YOU TRY IT: Find the domain and range of the quadratic given below.

220.

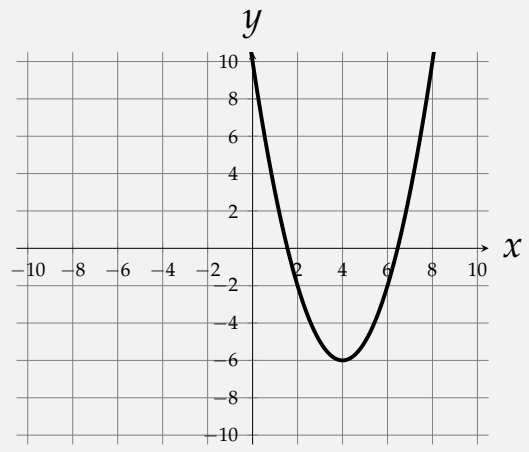




\section{How the leading coefficient affects the shape of the parabola}

$\square$ Watch Video 6: Investigating the Graphs of Quadratic Functions (vertical stretch and shrink) to complete the following.

Graph the functions defined below on the same graph.

\begin{tabular}{|l|l|l|l|}
\hline$x$ & $f(x)$ & $g(x)$ & $h(x)$ \\
\hline & & & \\
\hline & & & \\
\hline & & & \\
\hline & & & \\
\hline & & & \\
\hline & & & \\
\hline & & & \\
\hline
\end{tabular}

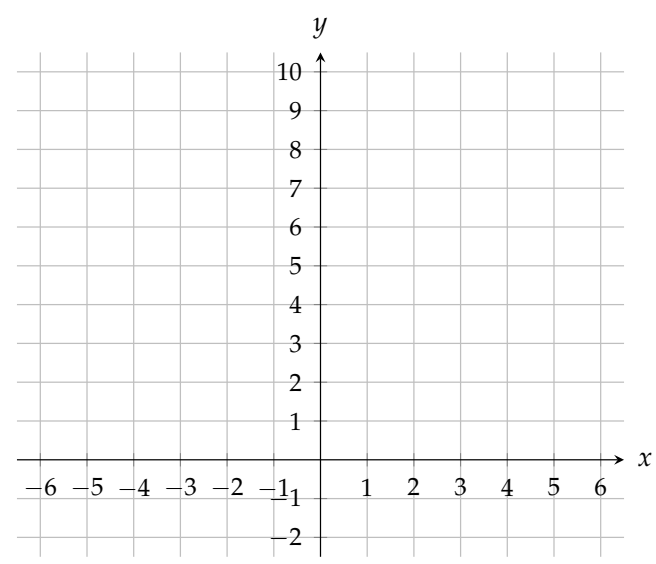

If we make a conjecture, we might think that the graph of $y=a x^{2}$

Graph the functions defined below on the same graph.

\begin{tabular}{|l|l|l|l|}
\hline$x$ & $f(x)$ & $g(x)$ & $h(x)$ \\
\hline & & & \\
\hline & & & \\
\hline & & & \\
\hline & & & \\
\hline & & & \\
\hline & & & \\
\hline & & & \\
\hline
\end{tabular}

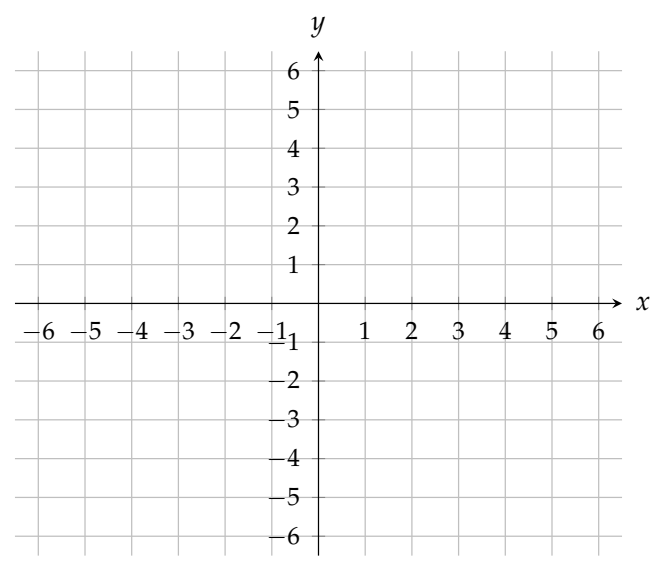

If we make a conjecture, we might think that the graph of $y=a x^{2}$ is a reflection over the $x$-axis for

The reflection of $y=x^{2}$ will stretch if $a<-1$ and shrink if $-1<a<0$. 
Finding the vertex, intercepts, and axis of symmetry from the graph of a parabola

Learning Page A parabola is a graph such as those shown below.

Here are some facts about parabolas. On each graph label the vertex and the axis of symmetry.

\begin{tabular}{|c|c|c|}
\hline \multicolumn{2}{|c|}{ Parabola opening upward } & Parabola opening downward \\
\hline The vertex is the on the graph. & The vertex is the on the graph. \\
\hline into & (This line goes through the $\_$is the line that the divides the parabola \\
\hline
\end{tabular}

\section{Finding the $x$-intercept(s) and the vertex of a parabola}

\section{Learning Page Finding the $x$-intercept(s)}

An $x$-intercept is the of a point where the graph

A parabola can have , or $x$-intercepts.

At each point where a graph crosses the $x$-axis, the

To find any $x$-intercepts of the parabola we let and solve the resulting quadratic equation.

$$
\begin{aligned}
& y= \\
& 0= \\
& 0=
\end{aligned}
$$

solution:

So the two $x$-intercepts are

\section{Continued on the next page}


$\triangleright \quad$ Watch the video Exercise: Determining the Vertex of a Quadratic Function to complete the following.

\section{The Vertex Formula}

For $f(x)=a x^{2}+b x+c(a \neq 0)$ the vertex is given by

$$
\left(\frac{-b}{2 a}, f\left(\frac{-b}{2 a}\right)\right)
$$

Find the vertex by using the vertex formula. $\quad r(x)=$

$x$-coordinate of the vertex:

$y$-coordinate of the vertex:

Vertex:

EXAMPLE: Find the vertex of

$$
f(x)=-2 x^{2}-16 x-40
$$

by using the vertex formula.

$$
\begin{gathered}
\frac{-b}{2 a}=\frac{-(-16)}{2(-2)} \\
=\frac{16}{-4}=-4 \\
f(-4)=-2(-4)^{2}-16(-4)-40 \\
=-32+64-40=-8
\end{gathered}
$$

So the vertex is $(-4,-8)$.

\section{YOU TRY IT:}

221. Find the vertex of $g(x)=2 x^{2}-4 x-9$ using the vertex formula. 
Graphing a parabola of the form $y=x^{2}+b x+c$

ใ्? Open the Instructor Added Resource which will direct you to a video to complete the following.

Sketch the graph of

Vertex:

$x$-coordinate:

$y$-coordinate:

$x$-intercepts:
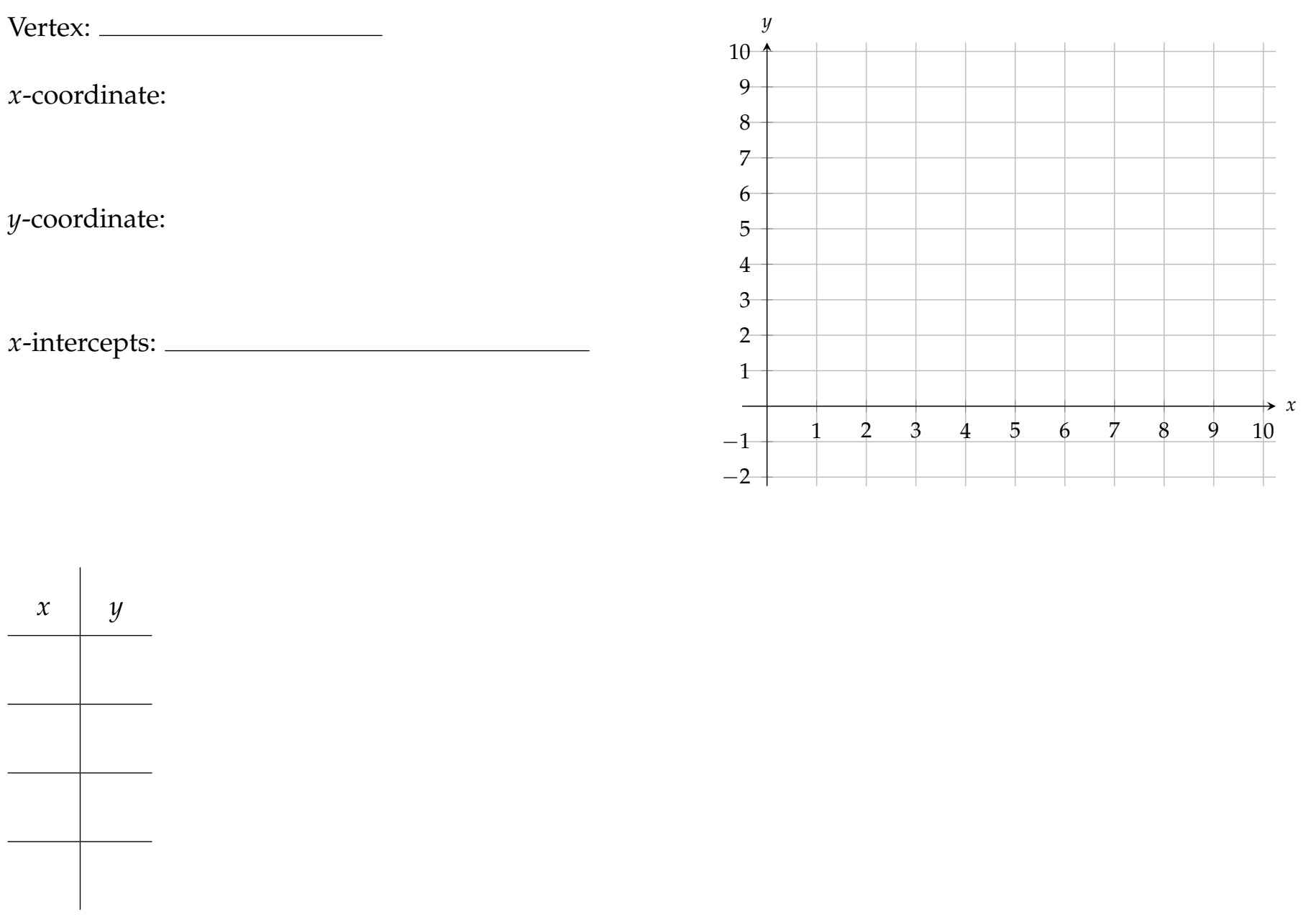


\section{Graphing a parabola of the form $y=a x^{2}+b x+c$ : Integer coefficients}

$\square$ Watch Video 3: Applying the Vertex Formula and Graphing a Quadratic Function to complete the following. NOTE: This may not be the first video that pops up. Select this video from the list of videos on the left of the video box.

FORMULA The Vertex Formula For $f(x)=a x^{2}+b x+c(a \neq 0)$ the vertex is given by

$$
\left(\frac{-b}{2 a}, \frac{4 a c-b^{2}}{4 a}\right) \text { or }
$$

Given $f(x)=$

$a=$

$b=$

$c=$

a. Use the vertex formula to find the vertex.

c. Determine the $x$-intercept(s).

b. Determine the $y$-intercept.

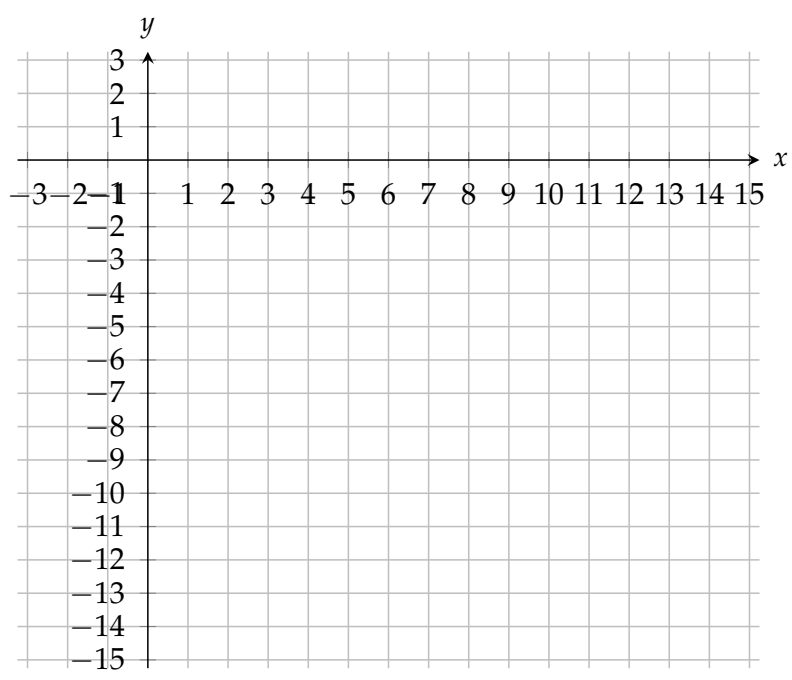

Notes from Focus Group: 
Notes from Focus Group: 


\section{Module 15-Final Review}

To help you review for your upcoming final exam, this module contains all of the topics from the course. Topics that you have already mastered will not appear in your carousel.

- ALEKS final exam

- The ALEKS final exam must be taken in the MALL.

- The ALEKS final exam is a Comprehensive Knowledge Check.

- The ALEKS final exam must be completed by

- To study for the final exams:

- Complete this ALEKS Final Review Module.

- Rework the problems on your old exams.

- Review your old Focus Group assignments. 


\section{Solutions}

Module 1

1. $17^{\circ} \mathrm{C}$

2. 126

3. 52

4. $-\frac{1}{3}$

5. $-\frac{21}{4}$

6. undefined

7. 0 (zero)

8. 36

9. -36

10. 125

11. $-\frac{1}{125}$

12. -11

13. 2

14. Associative Property of Addition

15. Distributive Property

16. Commutative Property of Multiplication

17. $-12 x+15$

18. $21 x+14$

19. $2 x+17 y$

20. $2 x+3 y$
21. $y=\frac{31}{35}$

22. $y=-\frac{15}{4}$

23. $y=-2$

24. $x=21$

25. $x=\frac{27}{4}$

26. $x=21$

27. $7+\frac{d}{6}=9$

28. $t-2$

\section{Module 2}

29. $x<2$

30.

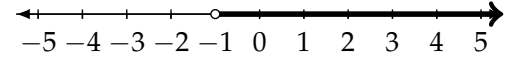

31.

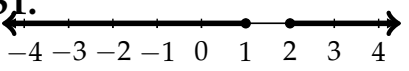

32. All numbers.

$\begin{array}{llllllll}\longleftrightarrow & \longleftrightarrow-4-3-2-1 & 0 & 1 & 2 & 3 & 4 & 5\end{array}$

33. 13

34. $x=A+y-12$

35. $\frac{A}{7}-4=x$

36. $x<5$

37. $x \geq \frac{7}{8}$
38. $x>0$ and $x \leq 4$

39. $(-\infty, 2]$

40. $x<-63$

41. $x \leq-\frac{32}{3}$

42. $(-3,-2$

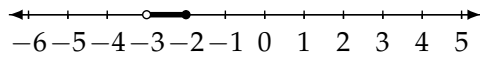

43. $(-\infty,-3] \cup(-2, \infty)$

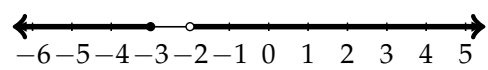

44. $\{3,-3\}$

45. No solution

46. $\{7,-7\}$

47. All real numbers

\section{Module 3}

48.

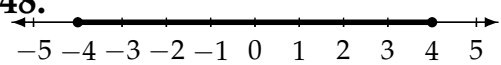

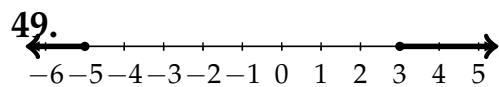

50. $|x|<1$

51. no

52. no

53. yes 
54.

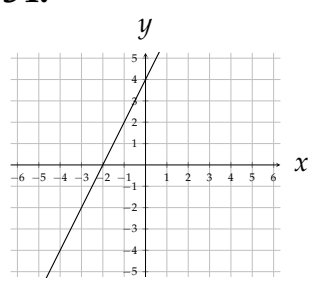

55.

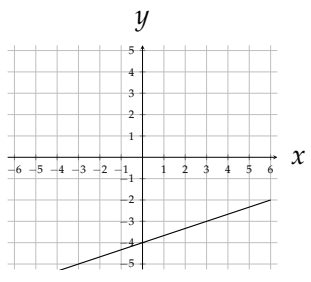

56.

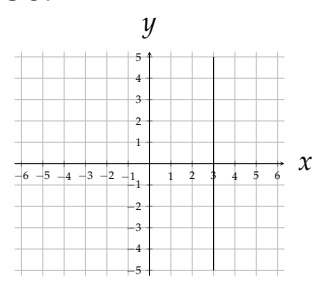

57.

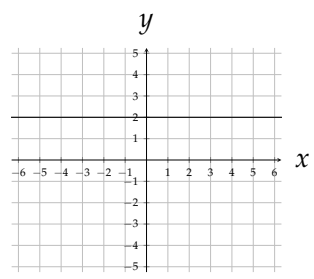

58. $(-3,0),(2,0),(0,-2)$

59. $m=\frac{3}{4}$

60. $m$ is undefined

61.

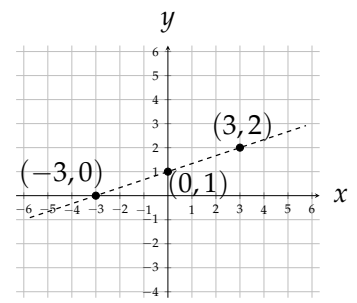

62.

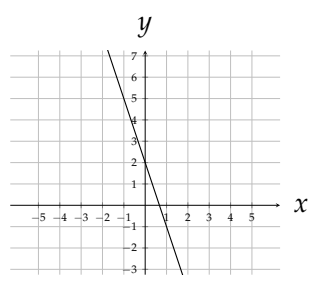

$m=-3$

$y$-intercept: $(0,2)$

63. slope: -2

$y$-intercept: $(0,4)$

64. Slope-intercept: $y=3 x+1$ Standard Form: $-3 x+y=1$

65. vertical line: $x=3$ horizontal line: $y=7$

66. $y=-\frac{3}{5} x-\frac{11}{5}$

67.
\begin{tabular}{|c|c|}
\hline$x$ & $f(x)$ \\
\hline-2 & -3 \\
\hline-1 & -1 \\
\hline 0 & 1 \\
\hline 1 & 3 \\
\hline 2 & 5 \\
\hline
\end{tabular}

68. domain: $\{2,-5,0,5\}$ range: $\{3,1,-4\}$

Module 5

69. domain: $(-\infty, \infty)$ range: $[-2, \infty)$

70. $[-100, \infty)$

71. $(-\infty, 100]$

72. Function

73. Not a Function

74. -31

75. $g(1)=3$

76. $x=0$

77. $(2,4)$ is a solution.

78.

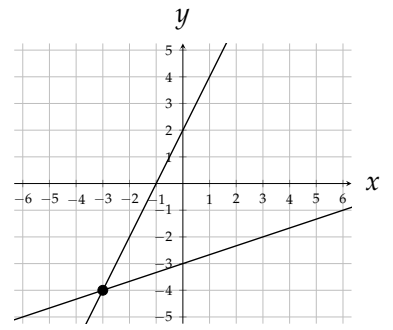

The solution set is $\{(-3,-4)\}$
79. $\{(-2,1)\}$

80. $(-2,2)$

81. $x=61^{\circ}, y=58^{\circ}$

82. notepad: $\$ 1.85$ marker: $\$ 0.65$

\section{Module 6}

83. $p^{9}$

84. $-8 x^{8}$

85. $12 x^{8} y^{11}$

86. $x^{30}$

87. $81 p^{4}$

88. $y^{6}$

89. $\frac{1}{y^{6}}$

90. $\frac{b^{2}}{a^{2}}$

91. $9 x^{2} y^{8}$

92. $-\frac{8 y^{12}}{x^{6}}$

93. $\frac{5 x}{4}$

94. -1

95. 1

96. $\frac{7}{8}$

97. $-\frac{8}{125}$

98. $-2 x^{8}$

99. $\frac{1}{x^{6}}$

100. $60 c^{3}$

101. $\frac{1}{x^{3}}$

102. $\frac{1}{x^{15}}$

103. $y^{42}$

Module 7 
104. $4 x^{5}-6 x^{3}+14$

105. $-10 x^{5}+35 x^{4}-30 x^{3}$

106. $-8 x^{4} y^{9} z^{5}+2 x^{8} y^{8} z$

107. $x^{2}+2 x-15$

108. $6 x^{2}+x-15$

109. $-6 x^{2}+19 x-15$

110. $3 x^{3}-5 x^{2}-17 x+15$

111. $9 x^{2}-16$

112. $9 x^{2}-12 x+4$

113. $x^{2}-2 x+\frac{3}{x}$

114. quotient: $2 x^{2}-3 x+13$ remainder: -36

115. quotient: $2 x^{2}+x+2$

remainder: -3

116. 7

117. $4 x^{2}$

118. $7 a b^{5}$

119. $7 x\left(2 x^{3}-x^{2}+3\right)$

120. $(y+2)\left(y^{3}-y-9\right)$

121. $(y+3)\left(y^{2}-3\right)$

122. $(y-4)\left(2 y^{2}-1\right)$

Module 9

123. $(x-9)(x-3)$

124. $-(x-3)(x+1)$ or $(3-$ $x)(x+1)$

125. $3(a+4)(2 a-1)$

126. $6\left(2 x^{2}+x+3\right)$

127. $(2 x+3)(x-5)$

128. $(x-5)^{2}$
129. Not a perfect square trinomial. Does not factor.

130. $-2 x(5 x-1)(2 x-3)$

131. $(x-7)(x+7)$

132. $x=-5, \frac{3}{2}$

133. $x=3,-7$

134. $x=-3,-5$

135. $x=0,5$

136. $x=-\frac{3}{4}, 1$

137. $x=\frac{1}{3},-\frac{2}{3}$

138. $x=-3,-2$

139. 6 in $X 13$ in

140. $-x^{2}-5 x-14=0$

Module 10

141. 1

142. Undefined

143. $-\frac{7}{4}$

144. 2

145. $\frac{4(x-7)}{9(x+7)}$

146. 2

147. $\frac{x+2}{2-x}$

148. -2

149. $\frac{x+1}{3}$

150. $-\frac{5}{x-6}$

151. $\frac{3}{7 a b}$

152. $-\frac{15}{2}$

153. $\frac{7 x}{6}$

154. $\frac{x+4}{x-4}$
155. $60 x^{2} y^{3} z$

156. $\frac{4 w^{6}}{14 w^{5}}$

157. $2 \cdot(3 x-8) \cdot 3 x=6 x(3 x-$ 8)

158. $\frac{11 x}{3 x-1}$

159. $\frac{x-11}{(x-1)(x+4)}$

160. $\frac{10+21 b^{3}}{12 b^{4}}$

161. $\frac{15}{2}$

\section{Module 11}

162. 7

163. $-\frac{5}{4}$

164. Not a real number

165. $a^{7}$

166. $7 y^{4}$

167. $4 x^{3}$

168. $28 a$

169. $\frac{1-15 x}{10 x+1}$

170. $x=-48$

171. $y=2$

172. $y=\frac{-1}{10}$

173. $x=-\frac{3}{2}, 1$

174. $y=-2,22$

175. $x=-13$

176. 5

177. -2

178. -6

Module 13

179. No real solution

180. -5

181. $3 x^{5}$

182. $2 x^{2}$ 
183. $x^{2 / 5}$

184. $y^{3 / 2}$

185. $\sqrt[3]{x^{5}}$

186. $\sqrt[7]{y^{2}}$

187. -3

188. -4

189. 9

190. Not a real number

191. $\frac{1}{x^{\frac{4}{5}}}$

192. $x^{\frac{1}{6}}$

193. $2 x^{4} \sqrt{6}$

194. $3 x^{5} \sqrt{2}$

195. $2 x^{5} \sqrt{5 x}$
196. $3 x^{7} \sqrt{3 x}$

197. $2 \sqrt[5]{4}$

198. $3 \sqrt{5}$

199. $10 \sqrt{3}$

200. $6 \sqrt{10}+8 \sqrt{2}$

201. $2 \sqrt{3 x}$

202. $6 \sqrt{2}-2 \sqrt{42}$

203. $72 \sqrt{3}$

204. $4 x^{3} \sqrt{30}$

Module 14

205. $\frac{3 \sqrt{7}}{7}$

206. No solution

207. $x=64$

208. No Solution
209. $x=10$

210. $V=\frac{b^{2} h}{3}$

211. $2 i \sqrt{6}$

212. $i \sqrt{15}$

213. $x= \pm 6$

214. $x= \pm \sqrt{5}$

215. $x= \pm 2 \sqrt{10}$

216. $\pm i \sqrt{2}$

217. $x=2 \pm \sqrt{10}$

218. $\frac{3 \pm \sqrt{5}}{2}$

219. $x=-\frac{1}{3} \pm i \frac{\sqrt{2}}{3}$

220. Domain: $(-\infty, \infty)$

Range: $[-6, \infty)$

221. $(1,-11)$ 


\section{Index}

Adding rational expressions with common denominators and GCF factoring, 171

Adding rational expressions with denominators $a x^{n}$ and $b x^{m}, 174$

Adding rational expressions with linear denominators with common factors: Basic, 173

Adding rational expressions with linear denominators without common factors: Basic, 172

Addition and subtraction of 3 fractions involving signs, 28

Additive property of equality with signed fractions, 35

Additive property of inequality with signed fractions, 51

Algebraic symbol manipulation with radicals, 220

Applying the quadratic formula: Exact answers, 225

Classifying slopes given graphs of lines, 76

Classifying the graph of a function, 105

Complex fraction made of sums involving rational expressions: Problem type 1, 182

Complex fraction without variables: Problem type 1, 175

Complex fraction without variables: Problem type 2, 176

Complex fraction: GCF factoring, 181

Converting between radical form and exponent form, 200

Cube root of an integer, 191

Degree and leading coefficient of a univariate polynomial, 125

Distributive property: Integer coefficients, 34

Dividing a polynomial by a monomial: Univariate, 132

Dividing rational expressions involving linear expressions, 167

Dividing rational expressions involving quadratics with leading coefficients of 1,168

Division involving zero, 30

Domain and range from ordered pairs, 83

Domain and range from the graph of a continuous function, 89

Domain and range from the graph of a parabola, 227

Domain of a square root function: Basic, 90

Estimating a square root, 191

Evaluating a quadratic expression: Integers, 50

Evaluating a rational function: Problem type 1, 160

Evaluating an expression with a negative exponent: Negative integer base, 117

Evaluating an expression with a negative exponent: Positive fraction base, 118

Evaluating an expression with a negative exponent: Whole number base, 117

Evaluating expressions with exponents of zero, 116

Evaluating functions: Linear and quadratic or cubic, 91

Exponents and integers: Problem type 1, 31

Exponents and integers: Problem type 2, 31

Exponents and signed fractions, 32 
Factoring a difference of squares in one variable: Advanced, 151

Factoring a difference of squares in one variable: Basic, 150

Factoring a perfect square trinomial with leading coefficient 1, 148

Factoring a perfect square trinomial with leading coefficient greater than 1, 149

Factoring a product of a quadratic trinomial and a monomial, 149

Factoring a quadratic with a negative leading coefficient, 144

Factoring a quadratic with leading coefficient 1,144

Factoring a quadratic with leading coefficient greater than 1: Problem type 1, 146

Factoring a quadratic with leading coefficient greater than 1: Problem type 2, 146

Factoring a univariate polynomial by grouping: Problem type 1, 139

Factoring a univariate polynomial by grouping: Problem type 2, 140

Factoring out a binomial from a polynomial: GCF factoring, basic, 138

Factoring out a constant before factoring a quadratic, 145

Factoring out a monomial from a polynomial: Univariate, 138

Finding $n^{\text {th }}$ roots of perfect $n^{\text {th }}$ powers with signs, 198

Finding $x$ - and $y$-intercepts given the graph of a line given the equation: Basic, 74

Finding $x$ - and $y$-intercepts given the graph of a line on a grid, 72

Finding all square roots of a number, 190

Finding inputs and outputs of a function from its graph, 94

Finding outputs of a one-step function that models a real-world situation: Function notation, 93

Finding slope given two points on the line, 74

Finding the $n^{\text {th }}$ root of perfect $n^{\text {th }}$ power monomial, 199

Finding the $x$-intercept(s) and the vertex of a parabola, 229

Finding the roots of a quadratic equation of the form $a x^{2}+b x=0,153$

Finding the roots of a quadratic equation with leading coefficient 1,152

Finding the roots of a quadratic equation with leading coefficient greater than 1,154

Finding the slope and $y$-intercept of a line given its equation in the form $y=m x+b, 79$

Finding the slope of horizontal and vertical lines, 75

Finding the vertex, intercepts, and axis of symmetry from the graph of a parabola, 229

Graphically solving a system of linear equations, 96

Graphing a compound inequality on the number line, 48

Graphing a function of the form $f(x)=a x^{2}, 105$

Graphing a line by first finding its slope and y-intercept, 78

Graphing a line given its equation in slope-intercept form: Fractional slope, 71

Graphing a line given its equation in slope-intercept form: Integer slope, 70

Graphing a line through a given point with a given slope, 77

Graphing a linear inequality on the number line, 47

Graphing a parabola of the form $y=a x^{2}+b x+c$ : Integer coefficients, 232

Graphing a parabola of the form $y=x^{2}+b x+c, 231$

Graphing a square root function: Problem type 1, 194

Graphing a vertical or horizontal line, 71

Greatest common factor of 2 numbers, 135

Greatest common factor of three univariate monomials, 136

Greatest common factor of two multivariate monomials, 137

Hamburger Menu, 11

How the leading coefficient affects the shape of the parabola, 228

Identifying functions from relations, 91

Identifying solutions to a linear equation in two variables, 69 
Identifying solutions to a system of linear equations, 95

Interpreting the graphs of two functions, 106

Introduction to simplifying a radical expression with an even exponent, 192

Introduction to simplifying a sum or difference of radical expressions: Univariate, 209

Introduction to solving a radical equation, 216

Introduction to solving an absolute value equation, 57

Introduction to square root addition or subtraction, 207

Introduction to the LCM of two monomials, 169

Introduction to the power of a power rule of exponents, 111

Introduction to the power of a product rule of exponents, 112

Introduction to the product rule of exponents, 109

Introduction to the product rule with negative exponents, 120

Introduction to the quotient rule of exponents, 113

Least common multiple of 2 numbers, 26

Multiplication involving binomials and trinomials in one variable, 129

Multiplicative property of equality with signed fractions, 36

Multiplicative property of inequality with integers, 53

Multiplying a multivariate polynomial by a monomial, 127

Multiplying a univariate polynomial by a monomial with a negative coefficient, 126

Multiplying binomials with leading coefficients greater than 1, 128

Multiplying binomials with leading coefficients of 1, 127

Multiplying binomials with negative coefficients, 128

Multiplying conjugate binomials: Univariate, 130

Multiplying rational expressions involving multivariate monomials, 166

Multiplying rational expressions made up of linear expressions, 167

Order of operations with integers and exponents, 32

Plotting a point in the coordinate plane, 68

Polynomial long division: Type 1, 133

Polynomial long division: Type 2, 134

Power and quotient rules with positive exponents, 115

Power of a power rule with negative exponents, 122

Power rules with positive exponents: Multivariate products, 114

Product rule with negative exponents, 120

Product rule with positive exponents: Multivariate, 110

Product rule with positive exponents: Univariate, 110

Properties of real numbers, 33

Quotient of expressions involving exponents, 114

Quotient rule with negative exponents: Problem type 1, 121

Rational exponents: Non-unit fraction exponent with a whole number base, 202

Rational exponents: Power of a power rule, 205

Rational exponents: Product rule, 204

Rational exponents: Unit fraction exponents and bases involving signs, 201

Rational exponents: Unit fraction exponents and whole number bases, 201

Restriction on a variable in a denominator: Quadratic, 161

Rewriting an algebraic expression without a negative exponent, 119

Set-builder and interval notation, 49 
Signed fraction division, 29

Simplifying a higher root of a whole number, 207

Simplifying a product involving square roots using the distributive property: Basic, 209

Simplifying a product of radical expressions: Univariate, 212

Simplifying a quotient of square roots, 215

Simplifying a radical expression with an even exponent, 205

Simplifying a radical expression with an odd exponent, 206

Simplifying a ratio of factored polynomials: Linear factors, 162

Simplifying a ratio of linear polynomials: $1,-1$, and no simplification, 163

Simplifying a ratio of polynomials by factoring a quadratic with leading coefficient 1, 164

Simplifying a ratio of polynomials using GCF factoring, 163

Simplifying a ratio of polynomials: Problem type 1, 165

Simplifying a sum or difference of two univariate polynomials, 125

Simplifying the square root of a whole number greater than 100, 203

Simplifying the square root of a whole number less than 100, 203

Solving a compound linear inequality: Graph solution, basic, 54

Solving a compound linear inequality: Interval notation, 56

Solving a distance, rate, time problem using a system of linear equations, 104

Solving a linear equation with several occurrences of the variable: Variables on both sides and distribution, 38

Solving a proportion of the form $\frac{(x+a)}{b}=\frac{c}{d}, 188$

Solving a quadratic equation needing simplification, 155

Solving a quadratic equation using the square root property: Exact answers, advanced, 224

Solving a quadratic equation using the square root property: Exact answers, basic, 223

Solving a quadratic equation with complex roots, 226

Solving a radical equation that simplifies to a linear equation: One radical, advanced, 218

Solving a radical equation that simplifies to a linear equation: One radical, basic, 217

Solving a radical equation that simplifies to a quadratic equation: One radical, basic, 219

Solving a rational equation that simplifies to linear: Denominator $x+a, 185$

Solving a rational equation that simplifies to linear: Denominators $a, x$, or $a x, 183$

Solving a rational equation that simplifies to linear: Denominators $a x$ and $b x, 184$

Solving a rational equation that simplifies to quadratic: Denominator $x, 186$

Solving a rational equation that simplifies to quadratic: Proportional form, basic, 187

Solving a system of linear equations using elimination with addition, 99

Solving a system of linear equations using elimination with multiplication and addition, 100

Solving a system of linear equations using substitution, 97

Solving a two-step equation with integers, 37

Solving a two-step equation with signed fractions, 37

Solving a two-step linear inequality: Problem type 2, 52

Solving a value mixture problem using a system of linear equations, 103

Solving a word problem involving a sum and another basic relationship using a system of linear equations, 101

Solving a word problem using a quadratic equation with rational roots, 156

Solving a word problem using a system of linear equations of the form $A x+B y=C, 102$

Solving a word problem with two unknowns using a linear equation, 42

Solving an absolute value equation: Problem Type 1, 58

Solving an absolute value equation: Problem Type 2, 59

Solving an absolute value inequality: Problem Type 1, 65

Solving an absolute value inequality: Problem Type 2, 66

Solving an absolute value inequality: Problem Type 3, 67

Solving an equation of the form $x^{2}=a$ using the square root property, 222

Solving an equation written in factored form, 152 
Solving for a variable in terms of other variables in a rational equation: Problem type 1, 188

Solving for a variable in terms of other variables using addition or subtraction with division, 51

Solving for a variable in terms of other variables using addition or subtraction: Advanced, 50

Solving for a variable inside parentheses in terms of other variables, 51

Solving inequalities with no solution or all real numbers as solutions, 60

Square root addition or subtraction, 208

Square root multiplication: Advanced, 211

Square root multiplication: Basic, 210

Square root of a perfect square monomial, 181

Square root of a perfect square with signs, 88

Square root of a rational perfect square, 180

Squaring a binomial: Univariate, 131

Table for a linear function, 82

Table for a square root function, 192

Technical Support, 12

The Learning Carousel, 10

Translating a sentence into a multi-step equation, 39

Translating a sentence into a multi-step inequality, 61

Understanding the power rules of exponents, 111

Understanding the product rule of exponents, 109

Using $i$ to rewrite square roots of negative numbers, 222

Using distribution with double negation and combining like terms to simplify: Multivariate, 34

Variable expressions as inputs of functions: Problem type 1, 93

Vertical line test, 92

Word problem involving multiple rates, 193

Word problem involving radical equations: Advanced, 221

Word problem on proportions: Problem type 1, 189

Word problem on proportions: Problem type 2, 190

Word problem with addition or subtraction of integers, 26

Working in ALEKS with the Notebook, 10

Writing a compound inequality given a graph on the number line, 52

Writing a multi-step equation for a real-world situation, 40

Writing a one-step expression for a real-world situation, 41

Writing a quadratic equation given the roots and the leading coefficient, 157

Writing an absolute value inequality given a graph on the number line, 68

Writing an equation in slope-intercept form given the slope and a point, 79

Writing an inequality for a real-world situation, 46

Writing an inequality given a graph on the number line, 46

Writing equivalent rational expressions with monomial denominators, 169

Writing the equation of the line through two given points, 81

Writing the equations of vertical and horizontal lines through a given point, 80 


\section{ARITHMETIC PROPERTIES}

\begin{tabular}{|ll|ll|}
\hline Associative: & $\begin{array}{l}\text { addition: } a+(b+c)=(a+b)+c \\
\text { multiplication: } a(b c)=(a b) c\end{array}$ & Identity: & $\begin{array}{l}\text { addition: } 0+a=a \\
\text { multiplication: } 1 \cdot a=a\end{array}$ \\
\hline Commutative: & addition: $a+b=b+a$ & Inverse: & addition: $a+(-a)=0$ \\
& multiplication: $a b=b a$ & multiplication: $a \cdot \frac{1}{a}=1, a \neq 0$ \\
\hline Distributive: & $a(b+c)=a b+a c$ & & \\
\hline
\end{tabular}

\section{FRACTIONS}

\begin{tabular}{ll|ll}
\hline Adding: & $\frac{a}{b}+\frac{c}{d}=\frac{a d+b c}{b d}$ & Multiplying: & $\frac{a}{b} \cdot \frac{c}{d}=\frac{a c}{b d}$ \\
\hline Subtracting: & $\frac{a}{b}-\frac{c}{d}=\frac{a d-b c}{b d}$ & Dividing: & $\frac{a}{b} \div \frac{c}{d}=\frac{a}{b} \cdot \frac{d}{c}=\frac{a d}{b c}$
\end{tabular}

\section{FACTORING}

\begin{tabular}{|c|c|}
\hline Difference of Two Squares & Sum and Difference of Two Cubes \\
$a^{2}-b^{2}=(a-b)(a+b)$ & $a^{3}+b^{3}=(a+b)\left(a^{2}-a b+b^{2}\right)$ \\
$a^{2}+b^{2}=$ Does not factor & $a^{3}-b^{3}=(a-b)\left(a^{2}+a b+b^{2}\right)$ \\
\hline Perfect Square Trinomials & \\
$a^{2}-2 a b+b^{2}=(a-b)^{2}$ & \\
$a^{2}+2 a b+b^{2}=(a+b)^{2}$ & \\
\hline
\end{tabular}

\section{DISTANCE AND MIDPOINT FORMULAS}

Distance between $\left(x_{1}, y_{1}\right)$ and $\left(x_{2}, y_{2}\right)$

$$
d=\sqrt{\left(x_{2}-x_{1}\right)^{2}+\left(y_{2}-y_{1}\right)^{2}}
$$

Midpoint between $\left(x_{1}, y_{1}\right)$ and $\left(x_{2}, y_{2}\right)$

$$
m=\left(\frac{x_{1}+x_{2}}{2}, \frac{y_{1}+y_{2}}{2}\right)
$$

\begin{tabular}{|ll|ll|}
\hline \multicolumn{3}{|c|}{ ABSOLUTE VALUE } \\
\hline Statement & Equivalent Statement & Statement & Equivalent Statement \\
$|x|=a$ & $x=a$ or $x=-a$ & $|x| \leq a$ & $-a \leq x \leq a$ \\
$|x|=|y|$ & $x=y$ or $x=-y$ & $|x| \geq a$ & $x \leq-a$ or $x \geq a$ \\
\hline
\end{tabular}

\section{CIRCLE}

Standard Form of a Circle with center $(h, k)$ and radius $r:(x-h)^{2}+(y-k)^{2}=r^{2}$ 


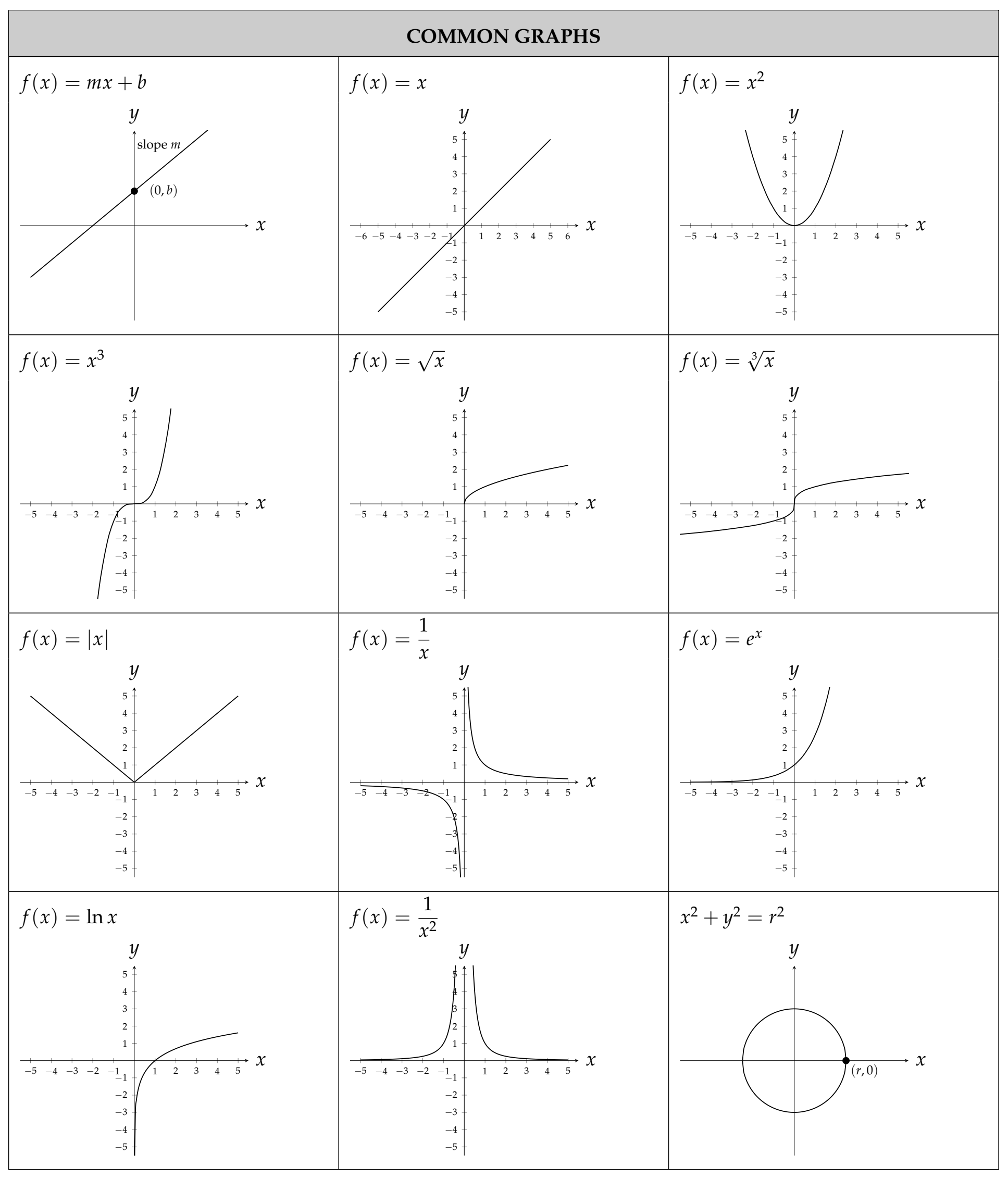


Common Properties, Graphs \& Formulas

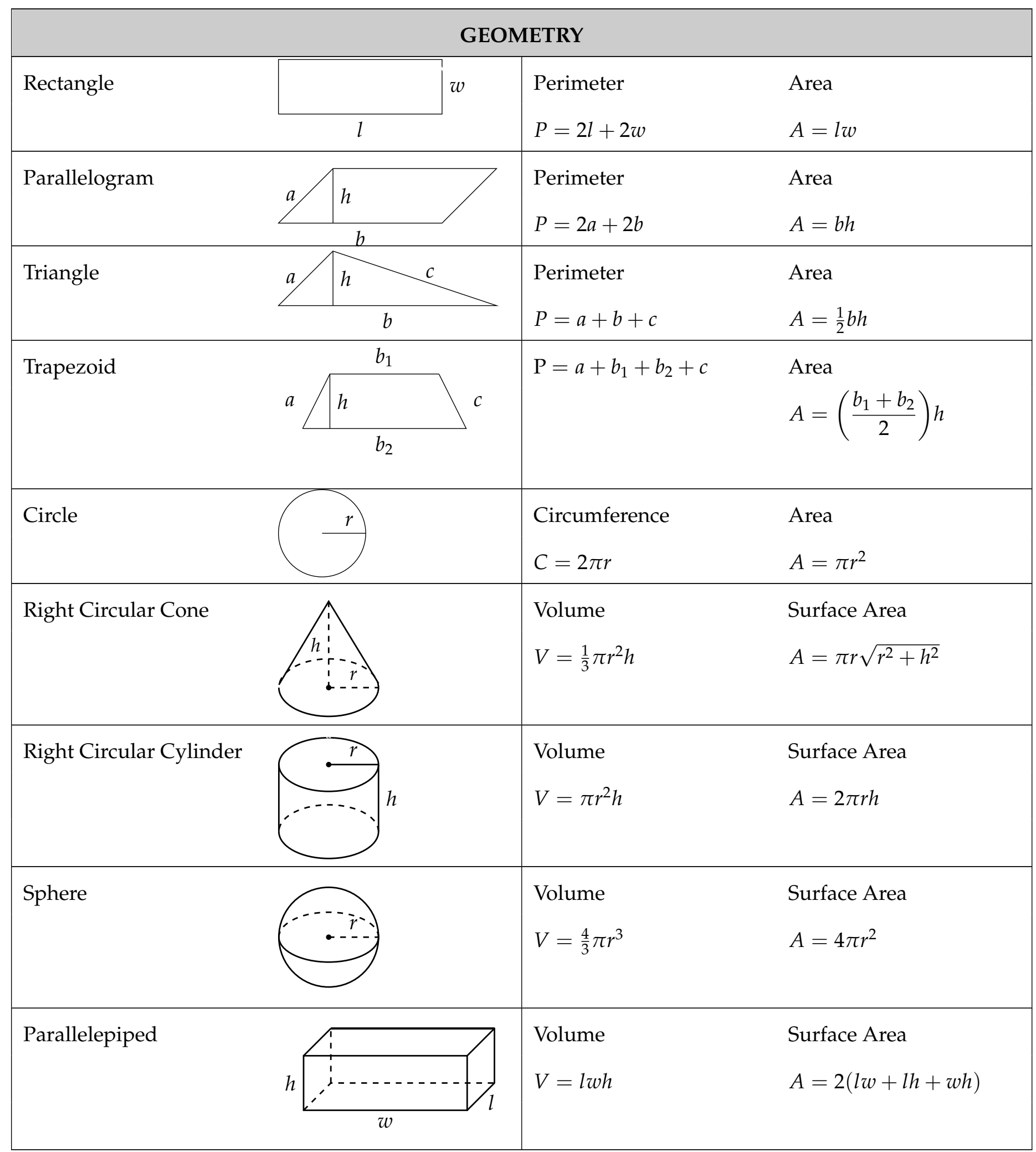




\section{Common Properties, Graphs \& Formulas}

\begin{tabular}{|c|c|}
\hline \multicolumn{2}{|c|}{ PROPERTIES OF EXPONENTS } \\
\hline $\begin{array}{ll}a^{m} \cdot a^{n}=a^{m+n} & \frac{a^{m}}{a^{n}}=a^{m-n} \\
a^{0}=1, a \neq 0 & a^{-n}=\frac{1}{a^{n}}\end{array}$ & $\begin{array}{l}\left(a^{n}\right)^{m}=a^{n m} \\
\left(\frac{a}{b}\right)^{n}=\frac{a^{n}}{b^{n}}\end{array}$ \\
\hline \multicolumn{2}{|c|}{ DEFINITION OF LOGARITHM } \\
\hline $\log _{a} x=y \Longleftrightarrow a^{y}=x$ & $\ln x=y \Longleftrightarrow e^{y}=x$ \\
\hline \multicolumn{2}{|c|}{ LAWS OF LOGARITHMS } \\
\hline $\log _{a} m+\log _{a} n=\log _{a} m n$ & $\ln m+\ln n=\ln m n$ \\
\hline $\log _{a} m-\log _{a} n=\log _{a} \frac{m}{n}$ & $\ln m-\ln n=\ln \frac{m}{n}$ \\
\hline $\log _{a} m^{n}=n \log _{a} m$ & $\ln m^{n}=n \ln m$ \\
\hline
\end{tabular}

